\title{
The Supermarket Model with Bounded Queue Lengths in Equilibrium
}

\author{
Graham Brightwell $^{1}$. Marianne Fairthorne ${ }^{1}$. \\ Malwina J. Luczak ${ }^{2}$ (D)
}

Received: 26 October 2017 / Accepted: 16 April 2018 / Published online: 28 April 2018

(C) The Author(s) 2018

\begin{abstract}
In the supermarket model, there are $n$ queues, each with a single server. Customers arrive in a Poisson process with arrival rate $\lambda n$, where $\lambda=\lambda(n) \in(0,1)$. Upon arrival, a customer selects $d=d(n)$ servers uniformly at random, and joins the queue of a least-loaded server amongst those chosen. Service times are independent exponentially distributed random variables with mean 1 . In this paper, we analyse the behaviour of the supermarket model in the regime where $\lambda(n)=1-n^{-\alpha}$ and $d(n)=\left\lfloor n^{\beta}\right\rfloor$, where $\alpha$ and $\beta$ are fixed numbers in $(0,1]$. For suitable pairs $(\alpha, \beta)$, our results imply that, in equilibrium, with probability tending to 1 as $n \rightarrow \infty$, the proportion of queues with length equal to $k=\lceil\alpha / \beta\rceil$ is at least $1-2 n^{-\alpha+(k-1) \beta}$, and there are no longer queues. We further show that the process is rapidly mixing when started in a good state, and give bounds on the speed of mixing for more general initial conditions.
\end{abstract}

Keywords Supermarket model · Markov chains · Rapid mixing · Concentration of measure · Load balancing

The research of Malwina Luczak was supported by an EPSRC Leadership Fellowship, Grant references $\mathrm{EP} / \mathrm{J} 004022 / 1$ and EP/J004022/2.

$\bowtie$ Graham Brightwell

g.r.brightwell@1se.ac.uk

http://www.maths.lse.ac.uk/Personal/graham/

Marianne Fairthorne

marianne.fairthorne@googlemail.com

Malwina J. Luczak

mluczak@unimelb.edu.au

https://findanexpert.unimelb.edu.au/display/person450456

1 Department of Mathematics, London School of Economics, Houghton Street, London WC2A 2AE, UK

2 School of Mathematics and Statistics, University of Melbourne, Melbourne, Australia 


\section{Introduction}

The supermarket model is a well-studied Markov chain model for a dynamic load-balancing process. There are $n$ servers, and customers arrive according to a Poisson process with rate $\lambda=\lambda(n)<1$. On arrival, a customer inspects $d=d(n)$ queues, chosen uniformly at random with replacement, and joins a shortest queue among those inspected (in case of a tie, the first shortest queue in the list is joined). Each server serves one customer at a time, and service times are iid random variables, with an exponential distribution of mean 1.

A number of authors [5-9,11-13,17,18,21,23] have studied the supermarket model, as well as various extensions, e.g., to the setting of a Jackson network [15] and to a version with one queue saved in memory $[14,20]$. There are related ideas in other queueing models, for instance one where one server inspects $d$ queues and serves the longest [1].

Early papers on the supermarket model concentrated on the case where $\lambda$ and $d$ are held fixed as $n$ tends to infinity. As with other related models (see, e.g. [10,19]), there is a dramatic change when $d$ is increased from 1 to 2 : if $d=1$, the maximum queue length in equilibrium is of order $\log n$, while if $d$ is a constant at least 2, then the maximum queue length in equilibrium is of order $\log \log n / \log d$.

Luczak and McDiarmid [11] prove that, for fixed $\lambda$ and $d$, the sequence of Markov chains indexed by $n$ is rapidly mixing: as $n \rightarrow \infty$, the time for the system to converge to equilibrium is of order $\log n$, provided the initial state has not too many customers and no very long queue. Also, they show that, for $d \geq 2$, with probability tending to 1 as $n \rightarrow \infty$, in the equilibrium distribution the maximum queue length takes one of at most 2 values, and that these values are $\log \log n / \log d+O(1)$.

More recently, there has been interest in regimes where the parameters of the model may vary as $n$ tends to infinity. Fairthorne [6] and Mukherjee et al [21] treat the case where $\lambda<1$ is fixed and $d=d(n)$ tends to infinity with $n$. Eschenfeldt and Gamarnik [5] consider the "heavy traffic regime", where $\lambda=\lambda(n)$ tends to 1 from below as $n \rightarrow \infty$, and $d$ is held fixed.

In this paper, we study a different regime. We focus on the case where $\lambda=\lambda(n)=1-n^{-\alpha}$ and $d=d(n)=\left\lfloor n^{\beta}\right\rfloor$, where $\alpha$ and $\beta$ are fixed constants in $(0,1]$ with $k-1<\alpha / \beta<k$ for some positive integer $k$. We also require that $2 \alpha<1+\beta(k-1)$, for reasons that we shall explain after the statement of Theorem 1.1 (see Remark (4)). Our results imply that, in equilibrium, with high probability (i.e., with probability tending to 1 as $n \rightarrow \infty$ ), the proportion of queues of length exactly equal to $k$ is at least $1-2 n^{-\alpha+(k-1) \beta}$, and there are no longer queues. Our methods actually cover a much broader range of parameter values, but we focus on this case for ease of exposition.

We offer two reasons why such a regime might be of interest: for one, this is a range of parameter values where near-perfect load balancing is achieved, with bounded maximum queue length, even when the system is running at nearly full capacity, and the values of $d$ we obtain thus represent a sufficient amount of resource (in terms of inspection of queuelengths) required to achieve this load-balancing. From a more theoretical viewpoint, we see our regimes, for the different values of $\lceil\alpha / \beta\rceil$, as possessing a scaling limit as $n \rightarrow \infty$, and varying the parameters so that $\alpha / \beta$ passes through an integer is an example of a phase transition.

To motivate our results, we first give heuristics to indicate what behaviour we might expect. Consider the infinite system of differential equations

$$
\frac{d v_{j}(t)}{d t}=\lambda\left(v_{j-1}(t)^{d}-v_{j}(t)^{d}\right)-\left(v_{j}(t)-v_{j+1}(t)\right), \quad j \geq 1,
$$


where $v_{0}(t)=1$ for all $t$. For an initial condition $v(0)$ such that $1 \geq v_{1}(0) \geq v_{2}(0) \geq \cdots \geq 0$ and $v_{j}(0) \rightarrow 0$ as $j \rightarrow \infty$, there is a unique solution $v(t)(t \geq 0)$, with $v(t)=\left(v_{j}(t)\right)_{j \geq 1}$, which is such that $1 \geq v_{1}(t) \geq v_{2}(t) \geq \cdots \geq 0$ and $v_{j}(t) \rightarrow 0$ as $j \rightarrow \infty$, for each $t \geq 0$. It follows from earlier work $[7,8,12,13,23]$ that, with high probability, for each $j$, the proportion of queues of length at least $j$ at time $t$ stays "close to" $v_{j}(t)$ over a bounded time interval (or an interval whose length tends to infinity at most polynomially with $n$ ), assuming this is the case at time 0 .

The system (1.1) has a unique, attractive, fixed point $\pi=\left(\pi_{j}\right)_{j \geq 1}$, such that $\pi_{j} \rightarrow 0$ as $j \rightarrow \infty$, given by

$$
\pi_{j}=\lambda^{1+\cdots+d^{j-1}}, \quad j \geq 1 .
$$

If $\lambda$ and $d$ are fixed constants, then, in equilibrium, with high probability, the proportion of queues of length at least $j$ is close to $\pi_{j}$ for each $j \geq 1$; see $[7,8,11,12]$.

For $\lambda$ and $d$ functions of $n$, there is no single limiting differential equation (1.1), but rather a sequence of approximating differential equations, each with their own solutions and fixed points. In this paper, we do not address the question of whether such approximations to the evolution of the process are valid in generality, focussing solely on equilibrium behaviour and the time to reach equilibrium. If $\lambda=1-n^{-\alpha}$ and $d=\left\lfloor n^{\beta}\right\rfloor$, and $k$ is an integer with $k-1<\alpha / \beta<k$, then

$$
\begin{aligned}
\pi_{k}=\lambda^{1+\cdots+d^{k-1}} & \geq\left(1-n^{-\alpha}\right)^{(1+o(1)) d^{k-1}} \geq 1-(1+o(1)) n^{-\alpha+(k-1) \beta} \\
& =1-o(1) \\
\pi_{k+1}=\lambda^{1+\cdots+d^{k}} & \leq \exp \left(-d^{k} n^{-\alpha}\right) \leq \exp \left(-\frac{1}{2} n^{k \beta-\alpha}\right)=o(1 / n) .
\end{aligned}
$$

We will indeed show that, in equilibrium, with high probability, there are no queues of length greater than $k$, while the proportion of queues with length exactly $k$ tends to 1 as $n \rightarrow \infty$. Moreover we show that, for $0 \leq j<k$, the number of queues of length exactly $j$ is very close to $n\left(\pi_{j}-\pi_{j+1}\right) \simeq n^{1-\alpha+j \beta}$.

We also prove results on mixing time to equilibrium. We show that, if we start in a "good" initial state (one without any very long queue, and without too many customers in the system in total), then the mixing time is of order $n^{1+(k-1) \beta} \log n$, which is best possible up to the logarithmic term. We also prove general bounds on the mixing time, in terms of the initial number of customers and the initial maximum queue length, and show that these bounds are also roughly best possible.

We will shortly state our main results precisely, but first we describe the supermarket model more carefully. In fact, we describe a natural discrete-time version of the process, which we shall work with throughout; as is standard, one may convert results about the discrete time version to the continuous model, with the understanding that one unit of time in the continuous model corresponds to about $(1+\lambda) n$ steps of the discrete model.

A queue-lengths vector is an $n$-tuple $(x(1), \ldots, x(n))$ whose entries are non-negative integers. If $x(j)=i$, we say that queue $j$ has length $i$, or that there are $i$ customers in queue $j$; we think of these customers as in positions $1, \ldots, i$ in the queue. We use similar terminology throughout; for instance, to say that a customer arrives and joins queue $j$ means that $x(j)$ increases by 1 , and to say that a customer in queue $j$ departs or is served means that $x(j)$ decreases by 1 . Given a queue-lengths vector $x$, we write $\|x\|_{1}=\sum_{j=1}^{n} x(j)$ to denote the total number of customers in state $x$, and $\|x\|_{\infty}=\max x(j)$ to denote the maximum queue length in state $x$.

For each $i \geq 0$, and each $x \in \mathbb{Z}_{+}^{n}$, we define $u_{i}(x)$ to be the proportion of queues in $x$ with length at least $i$. So $u_{0}(x)=1$ for all $x$, and, for each fixed $x$, the $u_{i}(x)$ form a 
non-increasing sequence of multiples of $1 / n$, such that $u_{i}(x)=0$ eventually. The sequence $\left(u_{i}(x)\right)_{i \geq 0}$ captures the "profile" of a queue-lengths vector $x$, and we shall describe various sets of queue-lengths vectors, and functions of the queue-lengths vector, in terms of the $u_{i}(x)$.

For positive integers $n$ and $d$, and $\lambda \in(0,1)$, we now define the $(n, d, \lambda)$-supermarket process. This process is a discrete-time Markov chain $\left(X_{t}\right)$, whose state space is the set $\mathbb{Z}_{+}^{n}$ of queue-lengths vectors, and where transitions occur at non-negative integer times. Each transition is either a customer arrival, with probability $\lambda /(1+\lambda)$, or a potential departure, with probability $1 /(1+\lambda)$. If there is a potential departure, then a queue $K$ is selected uniformly at random from $\{1, \ldots, n\}$ : if there is a customer in queue $K$, then they are served and depart the system. If there is an arrival, then $d$ queues are selected uniformly at random, with replacement, from $\{1, \ldots, n\}$, and the arriving customer joins a shortest queue among those selected. To be precise, a $d$-tuple $\left(K_{1}, \ldots, K_{d}\right)$ is selected, and the customer joins queue $k=K_{j}$, where $j$ is the least index such that $x\left(K_{j}\right)$ is minimal among $\left\{x\left(K_{1}\right), \ldots, x\left(K_{d}\right)\right\}$.

For $x \in \mathbb{Z}_{+}^{n},\left(X_{t}^{x}\right)$ denotes a copy of the $(n, d, \lambda)$-supermarket process $\left(X_{t}\right)$ where $X_{0}=x$ a.s. Throughout, we let $\left(Y_{t}\right)$ denote a copy of the process in equilibrium. The processes depend on the parameters $(n, d, \lambda)$, but we suppress this dependence in the notation. Throughout, we use $\left(\mathcal{F}_{t}\right)$ to denote the natural filtration of the process $\left(X_{t}\right)$. We use the notation $\mathbb{P}(\cdot)$ freely to denote probability in whatever space we work in.

We now state our main results. First, we describe sets of queue-lengths vectors $\mathcal{N}(n, \alpha, \beta)$ : our aim is to prove that, for suitable values of $\alpha$ and $\beta$, with $d=\left\lfloor n^{\beta}\right\rfloor, \lambda=1-n^{-\alpha}$ and $n$ sufficiently large, an equilibrium copy of the $(n, d, \lambda)$-supermarket process is concentrated in the set $\mathcal{N}(n, \alpha, \beta)$.

For $\alpha, \beta \in(0,1]$, let $k=\lceil\alpha / \beta\rceil$, and let $\mathcal{N}(n, \alpha, \beta)$ be the set of all queue-lengths vectors $x$ such that: $u_{k+1}(x)=0$ and, for $1 \leq j \leq k$,

$$
\left(1-\frac{1}{\log n}\right) n^{-\alpha+(j-1) \beta} \leq 1-u_{j}(x) \leq\left(1+\frac{1}{\log n}\right) n^{-\alpha+(j-1) \beta} .
$$

So, for $x \in \mathcal{N}(n, \alpha, \beta)$, we have the following.

(a) There are no queues of length $k+1$ or greater.

(b) For $1 \leq j \leq k$, the number of queues of length less than $j$ is $n\left(1-u_{j}(x)\right)$, which lies between $\left(1 \pm \frac{1}{\log n}\right) n^{1-\alpha+(j-1) \beta}$.

(c) In particular, the number of queues of length less than $k$ is at most $\left(1+\frac{1}{\log n}\right) n^{1-\alpha+(k-1) \beta}=$ $o(n)$, and so the proportion of queues of length exactly $k$ tends to 1 as $n \rightarrow \infty$.

(d) For $1 \leq j \leq k-1$, the number of queues of length exactly $j$ is $n\left(u_{j}(x)-u_{j+1}(x)\right)$, which lies between $\left(1 \pm \frac{2}{\log n}\right) n^{1-\alpha+j \beta}$.

Theorem 1.1 Suppose that $\alpha, \beta \in(0,1]$ are constants with $k-1<\alpha / \beta<k$ for some natural number $k$, and that $2 \alpha<1+\beta(k-1)$. Suppose also that $\lambda=\lambda(n)=1-n^{-\alpha}$ and $d=d(n)=\left\lfloor n^{\beta}\right\rfloor$. Then, for $n$ sufficiently large, a copy $\left(Y_{t}\right)$ of the $(n, d, \lambda)$-supermarket process in equilibrium satisfies

$$
\mathbb{P}\left(Y_{t} \notin \mathcal{N}(n, \alpha, \beta)\right) \leq e^{-\frac{1}{4} \log ^{2} n} .
$$

Remarks (1) In fact, our proofs go through essentially unchanged if we demand only that $1-\lambda(n)=n^{-\alpha+\delta_{1}(n)}$ and $d(n)=n^{\beta+\delta_{2}(n)}$, where $\delta_{1}(n)$ and $\delta_{2}(n)$ tend to zero as $n \rightarrow \infty$, and we replace instances of $n^{-\alpha+(j-1) \beta}$ in the definition of $\mathcal{N}(n, \alpha, \beta)$ by $(1-\lambda) d^{j-1}$. For ease of exposition, we prefer to stick to definite values of $\lambda$ and $d$; however, from now on we allow ourselves to write simply $d=n^{\beta}$, even though this need not be an integer. 
(2) The conclusion of the theorem implies that it is rare for there to be queues of length greater than $k$ in equilibrium, and so in particular it is rare for the last arriving customer to have joined a queue containing $k$ other customers. Theorem 1.1 can thus be used to make statements about the performance of the system in equilibrium in terms of the total waiting time for each customer; we leave the details to the interested reader.

(3) In the case where $\alpha \leq \beta$, Theorem 1.1 tells us that, in equilibrium, the maximum queuelength is 1 with high probability, and therefore that it will be extremely rare for an arriving customer to join a non-empty queue. In this case, some of the complexity of our proof can be avoided. This range is also covered by Fairthorne [6], with essentially the same proof and some sharper results, e.g. giving conditions for the maximum queue-length remaining equal to 1 for a time period $n^{K}$ for fixed $K$.

(4) We now indicate why the condition $2 \alpha<1+\beta(k-1)$ in Theorem 1.1 is necessary. For a state in $\mathcal{N}(n, \alpha, \beta)$, the total number of customers in the system is at least $k n-$ $2 n^{1-\alpha+(k-1) \beta}$. If we consider the next $n^{2 \alpha}$ steps, the number of arrivals minus the number of potential departures is asymptotically a normal random variable with mean and standard deviation both of order $n^{\alpha}$. So the probability that the number of arrivals minus the number of departures is at least $3 n^{\alpha}$ is bounded away from zero as $n \rightarrow \infty$. If $\alpha \geq 1-\alpha+(k-1) \beta$, then this many excess arrivals would drive the total number of customers in the system over $k n$, which certainly implies that some queue of length $k+1$ would be created.

(5) If $\alpha \geq 1$ and $\beta$ is arbitrary, a similar argument shows that, in equilibrium, for each $k$, the probability that there is a queue of length at least $k$ is bounded away from zero. Indeed, starting from any state, for any $k \in \mathbb{N}$, there is a positive probability that, over the next $n^{2}$ transitions, the number of arrivals exceeds the number of departures by at least $k n$.

(6) For $\lambda<\lambda^{\prime}$, there is a coupling of the $(n, d, \lambda)$ - and $\left(n, d, \lambda^{\prime}\right)$-supermarket processes, so that at each time, each queue in the $(n, d, \lambda)$-supermarket process is no longer than in the $\left(n, d, \lambda^{\prime}\right)$-supermarket process, provided this is true at time 0 . So, for instance, if at a given time there are at least $m$ queues with length $k$ in the $(n, d, \lambda)$-supermarket process, then there are also at least $m$ queues with length at least $k$ in the $\left(n, d, \lambda^{\prime}\right)$-supermarket process. If $\alpha / \beta$ is equal to a positive integer $k$, and $\alpha<k /(k+1)$ (so that the condition $2 \alpha<1+(k-1) \beta$ is satisfied), then we can couple with the process for slightly lower, and slightly higher, values of $\alpha$, to see that the maximum queue length in equilibrium is, with high probability, either $k$ or $k+1$, and that most queues have length either $k$ or $k+1$. Similarly, for $d<d^{\prime}$, there is a coupling of the $\left(n, d^{\prime}, \lambda\right)$-supermarket process and the $(n, d, \lambda)$-supermarket process such that, for all times $t \geq 0$, and for each $j$, the number of customers in position at least $j$ in their queue is no higher in the first process than the second (see [7,22]).

Combining these arguments actually gives an essentially complete picture of the maximum queue length in equilibrium for any parameters $\alpha \in(0,1), \beta>0$. The regions of the $(\alpha, \beta)$-plane not covered by Theorem 1.1 are of the form $E_{k}=\{(\alpha, \beta): \alpha<$ $\left.1, \frac{\alpha}{k} \leq \beta \leq \frac{2 \alpha-1}{k-1}\right\}$. For a model with parameters in $E_{k}$, coupling in $d$ shows that, with high probability, the maximum queue length in equilibrium is at most $k+1$; coupling in $\lambda$ shows that, with high probability, the maximum queue length in equilibrium is at least $k$. Moreover, the argument in Remark (4) shows that the value $k+1$ occurs with probability bounded way from zero as $n \rightarrow \infty$.

(7) We define the model so that $d$ queues are chosen with replacement, so it makes sense to ask what happens if $\beta>1$. In this case, most arriving customers inspect every queue, and the situation is essentially the same as when $\beta=1$ (when most arriving customers inspect at least half of the queues), or as when every arriving customer inspects every 
queue (the "join the shortest queue" protocol). Our result in this case says that, for $\alpha<1 / 2$, the maximum queue length is 1 with high probability in equilibrium. For $\alpha \geq 1 / 2$, we are in the region $E_{1}$ defined in the previous remark: the maximum queue length is either 1 or 2 with high probability in equilibrium, and the value 2 occurs with probability bounded away from 0 . For the join the shortest queue protocol and $\lambda=1-c n^{-1 / 2}$, this situation is explored in detail by Eschenfeldt and Gamarnik [4].

(8) The case $\alpha=1 / 2$ has been studied in queueing theory under the name of the HalfinWhitt heavy traffic regime. In this case, Theorem 1.1 applies whenever $\beta<1 / 2$ and $1 / 2 \beta$ is not an integer, and the result implies that, in equilibrium, the proportion of queues of length $\lceil 1 / 2 \beta\rceil$ tends to 1 as $n \rightarrow \infty$, and with high probability there are no longer queues. For $\beta>1 / 2$, the maximum queue length in equilibrium is either 1 or 2 with high probability, and the value 2 occurs with probability bounded away from 0 , as in Remark (4).

This is an explicit example of a model where we have a type of scaling limit: as we increase $n$ with $\lambda=1-n^{-\alpha}$ and $d=n^{\beta}$, we retain the property that almost all queues have length $k=\lceil\alpha / \beta\rceil$ in equilibrium, with high probability, and the number of shorter queues is of order $n^{1-\alpha+\lfloor\alpha / \beta\rfloor \beta}=o(n)$. As we adjust the parameters so that $\alpha / \beta$ passes through an integer value, we have a phase transition to a different equilibrium regime.

As mentioned earlier, and explained in more detail in Sect. 2, our results are in line with a more general hypothesis: for a very wide range of parameter values, the maximum queue length of the $(n, d, \lambda)$-supermarket model in equilibrium is within 1 of the largest $k$ such that

$$
\pi_{k}=\lambda^{1+d+\cdots+d^{k-1}}>\frac{1}{n} .
$$

(Recall that $\pi_{k}$ is the "predicted" proportion of queues of length at least $k$; see (1.2).) This general hypothesis holds when $\lambda$ and $d$ are constants: see [11]. It is also valid for the range where $\lambda$ is fixed and $d \rightarrow \infty$ : see [6], and at least approximately when $\lambda \rightarrow 1$ and $d$ is fixed: see [5].

We now state our results concerning "rapid mixing", i.e., rapid convergence to equilibrium. For $x \in \mathbb{Z}_{+}^{n}$, let $\mathcal{L}\left(X_{t}^{x}\right)$ denote the law at time $t$ of the $(n, d, \lambda)$-supermarket process $\left(X_{t}^{x}\right)$ started in state $x$. Also let $\Pi$ denote the stationary distribution of the $(n, d, \lambda)$-supermarket process.

Theorem 1.2 Suppose that $\lambda(n)=1-n^{-\alpha}$ and $d(n)=n^{\beta}$, where $\alpha, \beta$ and $k=\lceil\alpha / \beta\rceil$ satisfy the conditions of Theorem 1.1. Let $x$ be a queue-lengths vector in $\mathcal{N}(n, \alpha, \beta)$. Then, for all sufficiently large $n$ and for all $t \geq 0$,

$$
d_{T V}\left(\mathcal{L}\left(X_{t}^{x}\right), \Pi\right) \leq n\left(2 e^{-\frac{1}{4} \log ^{2} n}+4 \exp \left(-\frac{t}{1600 k n^{1+(k-1) \beta}}\right)\right) .
$$

In other words, for a copy of the process started in a state in $\mathcal{N}(n, \alpha, \beta)$, the mixing time is at most of order $n^{1+(k-1) \beta} \log n=o\left(n^{1+\alpha}\right)=o\left(n^{2}\right)$. In fact, this upper bound on the mixing time is best possible up to the logarithmic factor: we show that mixing, starting from states in $\mathcal{N}(n, \alpha, \beta)$, requires order at least $n^{1+(k-1) \beta}$ steps.

Theorem 1.3 Suppose that $\lambda(n)=1-n^{-\alpha}$ and $d(n)=n^{\beta}$, where $\alpha, \beta$ and $k=\lceil\alpha / \beta\rceil$ satisfy the conditions of Theorem 1.1. For all sufficiently large $n$, there is a state $z \in \mathcal{N}(n, \alpha, \beta)$ such that, for $t \leq \frac{1}{8} n^{1+(k-1) \beta}$,

$$
d_{T V}\left(\mathcal{L}\left(X_{t}^{z}\right), \Pi\right) \geq 1-2 e^{-\frac{1}{4} \log ^{2} n} .
$$


From states not in $\mathcal{N}(n, \alpha, \beta)$, we cannot expect to have rapid mixing in general. For instance, suppose we start from a state $x$ with number of customers $\|x\|_{1} \geq k n$. The expected decrease in the number of customers at each step of the chain is at most $\frac{1-\lambda}{1+\lambda}$, so mixing takes at least of order $\left(\|x\|_{1}-k n\right)(1-\lambda)^{-1}=\left(\|x\|_{1}-k n\right) n^{\alpha}$ steps. Similarly, if we start with one long queue, of length $\|x\|_{\infty}>k$, then mixing takes at least of order $\left(\|x\|_{\infty}-k\right) n$ steps, to allow time for enough departures from the long queue. This shows that, for instance, if either $\|x\|_{1} \geq 2 k n$ or $\|x\|_{\infty}>2 k$, and

$$
t \leq \frac{1}{10} \max \left(\left\|x_{1}\right\| n^{\alpha},\|x\|_{\infty} n\right)
$$

then the total variation distance $d_{T V}\left(\mathcal{L}\left(X_{t}^{x}, \Pi\right)\right.$ is near to 1 . The next result gives an upper bound on the mixing time for $\left(X_{t}^{x}\right)$ in terms of $\|x\|_{1}$ and $\|x\|_{\infty}$, and shows that (1.3) is best possible up to the constant factor.

Theorem 1.4 Suppose that $\alpha$ and $\beta$ satisfy the hypotheses of Theorem 1.1, and let $x$ be any queue-lengths vector with $\|x\|_{\infty} \leq e^{\frac{1}{4} \log ^{2} n}$. Then for $n$ sufficiently large and

$$
t \geq 7200\left(k n^{1+\alpha}+\|x\|_{1} n^{\alpha}+\|x\|_{\infty} n\right),
$$

we have $d_{T V}\left(\mathcal{L}\left(X_{t}^{x}\right), \Pi\right) \leq 2 e^{-\frac{1}{5} \log ^{2} n}$.

In the case where the dominant term in the expression above is $k n^{1+\alpha}$, this result is not as sharp as that in Theorem 1.2, since $\alpha>(k-1) \beta$.

The supermarket model is an instance of a model whose behaviour has been comprehensively analysed even though there are an unbounded number of variables that need to be tracked - namely, the proportions $u_{i}\left(X_{t}\right)$. While what we achieve in this paper is similar to what is achieved by Luczak and McDiarmid in [11] for the case where $\lambda$ and $d$ are fixed as $n \rightarrow \infty$, only some of the techniques of that paper can be used here, as we now explain.

The proofs in [11] rely on a coupling of copies of the supermarket process where the distance between coupled copies does not increase in time. This coupling is, in particular, used to establish concentration of measure, over a long time period, for Lipschitz functions of the queue-lengths vector; this result is valid for any values of $(n, d, \lambda)$, and in particular in our setting. Fast coalescence of coupled copies, and hence rapid mixing, is shown by comparing the behaviour of the $(n, d, \lambda)$-process $(d \geq 2)$ with the $(n, 1, \lambda)$-process, which is easy to analyse. This then also implies concentration of measure for Lipschitz functions in equilibrium, and that the profile of the equilibrium process is well concentrated around the fixed point $\pi$ of the equations (1.1).

The coupling from [11] also underlies the proofs in the present paper. However, in our regime, comparisons with the $(n, 1, \lambda)$-process are too crude. Thus we cannot show that the coupled copies coalesce quickly enough, until we know something about the profiles of the copies, in particular that their maximum queue lengths are small. Our approach is to investigate the equilibrium distribution first, as well as the time for a copy of the process from a fairly general starting state to reach a "good" set of states in which the equilibrium copy spends most of its time. Having done this, we then prove rapid mixing in a very similar way to the proof in [11].

To show anything about the equilibrium distribution, we would like to examine the trajectory of the vector $u\left(X_{t}\right)$, whose components are the $u_{i}\left(X_{t}\right)$ for $i \geq 1$. This seems difficult to do directly, but we perform a change of variables and analyse instead a collection of just $k$ functions $Q_{1}\left(X_{t}\right), \ldots, Q_{k}\left(X_{t}\right)$. These are linear functions of $u_{1}\left(X_{t}\right), \ldots, u_{k}\left(X_{t}\right)$, with the property that the drift of each $Q_{j}\left(X_{t}\right)$ can be written, approximately, in terms of $Q_{j}\left(X_{t}\right)$ 
and $Q_{j+1}\left(X_{t}\right)$ only. Exceptionally, the drift of $Q_{k}\left(X_{t}\right)$ is written in terms of $Q_{k}\left(X_{t}\right)$ and $u_{k+1}\left(X_{t}\right)$ (which in fact is usually zero in equilibrium). The particular forms of the $Q_{j}$ are chosen by considering the Perron-Frobenius eigenvalues of certain matrices $M_{k}$ derived from the drifts of the $u_{j}(x)$. Making this change of variables allows us to consider one function $Q_{j}\left(X_{t}\right)$ at a time, and show that each in turn drifts towards its equilibrium mean (which is derived from the fixed point $\pi$ of (1.1)), and we are thus able to prove enough about the trajectory of the $Q_{j}\left(X_{t}\right)$ to show that, starting from any reasonable state, with high probability the chain soon enters a good set of states where, in particular, $u_{k+1}\left(X_{t}\right)=0$, and so the maximum queue length is at most $k$. We also show that, with high probability, the chain remains in this good set of states for a long time, which implies that the equilibrium copy spends the vast majority of its time in this set. The argument from [11] about coalescence of coupled copies can be used to show rapid mixing from this good set of states. The drift of the function $Q_{k}$ to its equilibrium is slower than that of any other $Q_{j}$, and its drift rate is approximately $n^{-1-(k-1) \beta}$, which is close to the spectral gap of the Markov chain $\left(X_{t}\right)$, and hence determines the speed of mixing in Theorem 1.2.

The structure of the paper is as follows. In Sect. 2, we expand on the discussion above, and motivate the definitions of the functions $Q_{j}: \mathbb{Z}_{+}^{n} \rightarrow \mathbb{R}$, which are fundamental to the proof. In Sect. 3, we give a number of results about the long-term behaviour of random walks with drifts, including several variants on results from [11]. In Sect. 4, we describe the key coupling from [11], and use it to prove some results about the maximum queue length and number of customers. In Sect. 5, we discuss in detail the drifts of the functions $Q_{j}$. The proof of Theorem 1.1 starts in Sect. 6, where we show how to derive a slightly stronger result from a sequence of lemmas. These lemmas are proved in Sects. 7-9. We prove our results on mixing times in Sect. 10.

Note this paper is heavily based on a manuscript [3] by the first and third named authors, placed on the arXiv in 2012, but not published in any other outlet. The present paper also incorporates results from the second author's $\mathrm{PhD}$ thesis [6]. The results proved in the present paper are in some sense weaker than those in [3] and [6], as, purely for the sake of exposition, we only treat the case where $1-\lambda(n)$ and $d(n)$ are powers of $n$, and state our results only in asymptotic form. In a more important sense, our results here are stronger, as they cover essentially best possible ranges of exponents; the key improvement in our methodology compared to [3] is that here we state and use Lemma 3.2 in a form where we get a stronger bound when a function on the state space stays the same with high probability at any step, allowing us to take proper account of the fact that the $Q_{j}$ for $j<k$ rarely change value. Our intention is to update [3] to incorporate these improvements in our more general setting.

\section{Heuristics}

In this section, we set out the intuition behind our results and proofs. As before, let $\left(Y_{t}\right)$ be an equilibrium copy of the $(n, d, \lambda)$-supermarket process. Guided by the results in $[6,11]$, we start by supposing that, for each $i \geq 1, u_{i}\left(Y_{t}\right)$ is well-concentrated around its expectation $u_{i}$, and seeing what that implies about the $u_{i}$. For a function $F$ defined on the state space, and a state $x$, we define the drift of $F$ at $x$ to be $\Delta F(x)=\mathbb{E}\left[F\left(X_{t+1}\right)-F\left(X_{t}\right) \mid X_{t}=x\right]$, which is independent of $t$. We have

$$
\begin{aligned}
\Delta u_{i}\left(Y_{t}\right) & =\mathbb{E}\left[u_{i}\left(Y_{t+1}\right)-u_{i}\left(Y_{t}\right) \mid Y_{t}\right] \\
& =\frac{1}{n(1+\lambda)}\left[\lambda u_{i-1}\left(Y_{t}\right)^{d}-\lambda u_{i}\left(Y_{t}\right)^{d}-u_{i}\left(Y_{t}\right)+u_{i+1}\left(Y_{t}\right)\right] .
\end{aligned}
$$


To see this, observe that, for $i \geq 1$, conditioned on $Y_{t}$, the probability that the event at time $t+1$ is an arrival to a queue of length exactly $i-1$, increasing $u_{i}$ by $1 / n$, is $\frac{\lambda}{1+\lambda}\left(u_{i-1}\left(Y_{t}\right)^{d}-u_{i}\left(Y_{t}\right)^{d}\right)$, while the probability that the event is a departure from a queue of length exactly $i$, decreasing $u_{i}$ by $1 / n$, is $\frac{1}{1+\lambda}\left(u_{i}\left(Y_{t}\right)-u_{i+1}\left(Y_{t}\right)\right)$. Note that $u_{0}$ is identically equal to 1 .

Taking expectations on both sides, and setting them to 0 , we see that, since $\left(Y_{t}\right)$ is in equilibrium,

$$
0=\mathbb{E}\left[u_{i}\left(Y_{t+1}\right)-u_{i}\left(Y_{t}\right)\right] \simeq \frac{1}{n(1+\lambda)}\left[\lambda u_{i-1}^{d}-\lambda u_{i}^{d}-u_{i}+u_{i+1}\right],
$$

where the approximations $\mathbb{E} u_{i}\left(Y_{t}\right)^{d} \simeq u_{i}^{d}$ and $\mathbb{E} u_{i-1}\left(Y_{t}\right)^{d} \simeq u_{i-1}^{d}$ are justified because of our assumption that $u_{i}\left(Y_{t}\right)$ and $u_{i-1}\left(Y_{t}\right)$ are well-concentrated around their respective means $u_{i}$ and $u_{i-1}$.

The system of equations

$$
0=\lambda \pi_{i-1}^{d}-\lambda \pi_{i}^{d}-\pi_{i}+\pi_{i+1} \quad(i=1,2, \ldots),
$$

with $\pi_{0}=1$, has a unique solution with $\pi_{i} \rightarrow 0$ as $i \rightarrow \infty$, namely:

$$
\pi_{i}=\lambda^{1+\cdots+d^{i-1}} \quad(i=0,1, \ldots),
$$

as in (1.2). See [11] and the references therein for details.

By analogy with [11], and motivated by (2.2), if the $u_{i}\left(Y_{t}\right)$ are well concentrated, we expect that $u_{i} \approx \pi_{i}$, for each $i$, and moreover that the values of $u_{i}\left(Y_{t}\right)$ will be close to the corresponding $\pi_{i}$ with high probability. In the regime of Theorem 1.1,

$$
\log \pi_{i}=\log (1-(1-\lambda))\left(1+\cdots+d^{i-1}\right) \simeq-n^{-\alpha+(i-1) \beta},
$$

for each $i \geq 1$. As we are assuming that $(k-1) \beta<\alpha<k \beta$, this means that $\pi_{i}$ is close to 1 for $i \leq k$, and very close to 0 for $i>k$. In particular, $\pi_{k+1}$ (which we expect to be the approximate proportion of queues of length greater than $k$ ) is much smaller than $1 / n$, suggesting that, in equilibrium, the probability that there is a queue of length greater than $k$ is very small.

On the other hand, the fact that $\pi_{k}$ is close to 1 suggests that, in equilibrium, most queues have length exactly $k$. Moreover, $\pi_{i}^{d}=1-o(1)$ for $i<k$, so that $1-\pi_{i}^{d} \approx d\left(1-\pi_{i}\right)$, whereas $\pi_{k}^{d}=o(1)$. We then obtain the following linear approximation to the equations (2.3), written in terms of variables $1-\tilde{u}_{1}, \ldots, 1-\tilde{u}_{k}$ :

$$
\begin{aligned}
0= & \lambda d\left(1-\tilde{u}_{1}\right)+\left(1-\tilde{u}_{1}\right)-\left(1-\tilde{u}_{2}\right), \\
0= & -\lambda d\left(1-\tilde{u}_{i-1}\right)+\lambda d\left(1-\tilde{u}_{i}\right)+\left(1-\tilde{u}_{i}\right)-\left(1-\tilde{u}_{i+1}\right) \\
& (2 \leq i \leq k-1), \\
0= & -\lambda d\left(1-\tilde{u}_{k-1}\right)+\left(1-\tilde{u}_{k}\right)-(1-\lambda) .
\end{aligned}
$$

These linear equations have solution $\tilde{u}$ given by

$$
1-\tilde{u}_{i}=(1-\lambda)\left(1+(\lambda d)+\cdots+(\lambda d)^{i-1}\right) \quad(i=1, \ldots, k) .
$$

We then have the further approximation

$$
1-\tilde{u}_{i} \approx(1-\lambda)(\lambda d)^{i-1}, \quad(i=1, \ldots, k),
$$

and we aim to show that indeed each $u_{i}(x)$ is close to the corresponding $\tilde{u}_{i}$ with high probability in equilibrium. 
Ideally, we would seek a single "Lyapunov" function of the $u_{i}(x)$, which is small when $u_{i}(x) \approx \tilde{u}_{i}$ for each $i$, and larger otherwise, and which has a downward drift outside of a small neighbourhood of $\tilde{u}$ : we could then analyse the trajectory of this function to show that $\left(u_{1}(x), \ldots, u_{k}(x)\right)$ stays close to $\tilde{u}$ for a long period. We have been unable to find such a function, and indeed analysing the evolution of the $u_{i}\left(X_{t}\right)$ directly appears to be challenging. Instead, we work with a sequence of functions $Q_{j}(x), j=1, \ldots, k$, each of the form $Q_{j}(x)=n \sum_{i=1}^{j} \gamma_{j, i}\left(1-u_{i}(x)\right)$, where the $\gamma_{j, i}$ are positive real coefficients. This sequence of functions has the property that the drift of each $Q_{j}(x)$ can be written (approximately) in terms of $Q_{j}(x)$ itself and $Q_{j+1}(x)$.

Let us see how these coefficients should be chosen, starting with the special case $j=k$, where we write $\gamma_{i}$ for $\gamma_{k, i}$. Consider a function of the form $Q_{k}(x)=n \sum_{i=1}^{k} \gamma_{i}\left(1-u_{i}(x)\right)$. As in the argument leading to (2.1), we have that the drift of this function satisfies

$$
\begin{aligned}
(1+\lambda) \Delta Q_{k}(x)= & -(1+\lambda) n \sum_{i=1}^{k} \gamma_{i} \Delta u_{i}(x) \\
= & -\sum_{i=1}^{k} \gamma_{i}\left[\lambda u_{i-1}(x)^{d}-\lambda u_{i}(x)^{d}-u_{i}(x)+u_{i+1}(x)\right] \\
= & \sum_{i=1}^{k} \gamma_{i}\left[\lambda\left(1-u_{i-1}(x)^{d}\right)-\lambda\left(1-u_{i}(x)^{d}\right)\right. \\
& \left.-\left(1-u_{i}(x)\right)+\left(1-u_{i+1}(x)\right)\right] .
\end{aligned}
$$

Making the approximations $u_{k}(x)^{d} \simeq 0$, and $1-u_{i}(x)^{d} \simeq d\left(1-u_{i}(x)\right)$ for $i=1, \ldots, k-1$, and rearranging, we arrive at

$$
\begin{aligned}
(1+\lambda) \Delta Q_{k}(x) \simeq & \gamma_{k}\left(1-\lambda-u_{k+1}(x)\right)+\left(\gamma_{k-1}-\gamma_{k}\right)\left(1-u_{k}(x)\right) \\
& +\sum_{i=1}^{k-1}\left[\lambda d\left(\gamma_{i+1}-\gamma_{i}\right)-\gamma_{i}+\gamma_{i-1}\right]\left(1-u_{i}(x)\right)
\end{aligned}
$$

We set $\gamma_{0}=0$ for convenience of writing the above expression. This calculation is done carefully, with precise inequalities, in Lemma 5.1 below. We would like to choose the $\gamma_{i}$ so that the vector

$$
\left(\lambda d\left(\gamma_{2}-\gamma_{1}\right)-\gamma_{1}+\gamma_{0}, \ldots, \lambda d\left(\gamma_{k}-\gamma_{k-1}\right)-\gamma_{k-1}+\gamma_{k-2}, \gamma_{k-1}-\gamma_{k}\right)
$$

of coefficients of the $\left(1-u_{i}\right)$ in (2.4) is equal to some multiple $-\mu\left(\gamma_{1}, \ldots, \gamma_{k-1}, \gamma_{k}\right)$ of the vector with components $\gamma_{i}$, with $\mu>0$. This would entail

$$
(1+\lambda) \Delta Q_{k}(x) \simeq \gamma_{k}\left(1-\lambda-u_{k+1}(x)\right)-\frac{\mu Q_{k}(x)}{n},
$$

which in turn would mean that $Q_{k}$ drifts towards a value of $\gamma_{k}\left(1-\lambda-u_{k+1}(x)\right) n / \mu$. If also $u_{k+1}(x)$ is (nearly) equal to 0 , we should obtain that $Q_{k}(x)$ approaches $\gamma_{k}(1-\lambda) n / \mu-$ if $Q_{k}$ is above this value then it drifts down, whereas if $Q_{k}$ is below then it drifts up. What we need in order for the vector (2.5) to be a multiple of $\left(\gamma_{1}, \ldots, \gamma_{k}\right)$ is for $\left(\gamma_{1}, \ldots, \gamma_{k}\right)$ to be a left eigenvector of the $k \times k$ matrix 


$$
M_{k}=\left(\begin{array}{ccccccc}
-\lambda d-1 & 1 & 0 & \ldots & 0 & 0 & 0 \\
\lambda d & -\lambda d-1 & 1 & \ldots & 0 & 0 & 0 \\
0 & \lambda d & -\lambda d-1 & \ldots & 0 & 0 & 0 \\
\vdots & \vdots & \vdots & \ddots & \vdots & \vdots & \vdots \\
0 & 0 & 0 & \ldots & -\lambda d-1 & 1 & 0 \\
0 & 0 & 0 & \ldots & \lambda d & -\lambda d-1 & 1 \\
0 & 0 & 0 & \ldots & 0 & \lambda d & -1
\end{array}\right)
$$

with eigenvalue $-\mu$, or, equivalently, of the matrix

$$
M_{k}^{\prime}=M_{k}+(\lambda d+1) I_{k}=\left(\begin{array}{ccccccc}
0 & 1 & 0 & \cdots & 0 & 0 & 0 \\
\lambda d & 0 & 1 & \cdots & 0 & 0 & 0 \\
0 & \lambda d & 0 & \cdots & 0 & 0 & 0 \\
\vdots & \vdots & \vdots & \ddots & \vdots & \vdots & \vdots \\
0 & 0 & 0 & \cdots & 0 & 1 & 0 \\
0 & 0 & 0 & \cdots & \lambda d & 0 & 1 \\
0 & 0 & 0 & \cdots & 0 & \lambda d & \lambda d
\end{array}\right) .
$$

The non-negative matrix $M_{k}^{\prime}$ has a unique largest "Perron-Frobenius" eigenvalue, with a positive left eigenvector. By inspection, we see that, for $k \geq 2$, this left eigenvector is close to the all-1 vector, with an eigenvalue close to $\lambda d+1$, so that $M_{k}$ has largest eigenvalue very close to 0 . Recursion shows that a better approximation to the Perron-Frobenius left eigenvector of $M_{k}^{\prime}$ is $\left(\gamma_{1}, \ldots, \gamma_{k}\right)$, where

$$
\gamma_{i}=1-\frac{1}{(\lambda d)^{i}}-\frac{(i-1)}{(\lambda d)^{k}}
$$

for $i=1, \ldots, k$, and the largest eigenvalue $\mu$ of $M_{k}$ is very close to $-1 /(\lambda d)^{k-1}$. We shall see in Lemma 5.1 that this approximation is close enough for our purposes, enabling us to show that, with these choices of the $\gamma_{i}$,

$$
(1+\lambda) \Delta Q_{k}(x) \simeq(1-\lambda)-\frac{Q_{k}(x)}{n(\lambda d)^{k-1}},
$$

and thus $Q_{k}(x)$ drifts towards a value close to $(1-\lambda) n(\lambda d)^{k-1}$. A further consequence is that, in order for $Q_{k}(x)$ to move from $(1 \pm 2 \varepsilon)(1-\lambda) n(\lambda d)^{k-1}$ to $(1 \pm \varepsilon)(1-\lambda) n(\lambda d)^{k-1}$, it has to travel a distance of $\varepsilon(1-\lambda) n(\lambda d)^{k-1}$ while drifting at rate no greater than $2 \varepsilon(1-\lambda)$, and so time of order $n(\lambda d)^{k-1}$ is required. This is then a lower bound on the mixing time from a "good" state to equilibrium, nearly matching that in Theorem 1.2. We make this argument precise at the very end of the paper.

For $1 \leq j<k$, if $Q_{j}(x)=n \sum_{i=1}^{j} \gamma_{j, i}\left(1-u_{i}\right)$, then a similar analysis reveals that

$$
(1+\lambda) \Delta Q_{j}(x) \simeq \sum_{i=1}^{j}\left(1-u_{i}(x)\right)\left[\gamma_{j, i-1}+\lambda d \gamma_{j, i+1}-(\lambda d+1) \gamma_{j, i}\right]+\left(1-u_{j+1}(x)\right)
$$

(See the proof of Lemma 5.2.) We think of $1-u_{j+1}(x)$ as an "external" term (which in practice will be very close to $\left.Q_{j+1}(x) / n\right)$, which will determine the value towards which $Q_{j}$ drifts. We would like the rest of the expression to be a negative multiple of $Q_{j}(x)$. For this we need $\left(\gamma_{j, 1}, \ldots, \gamma_{j, j}\right)$ to be a left eigenvector of the $j \times j$ matrix 


$$
M_{j}=\left(\begin{array}{ccccccc}
-\lambda d-1 & 1 & 0 & \cdots & 0 & 0 & 0 \\
\lambda d & -\lambda d-1 & 1 & \cdots & 0 & 0 & 0 \\
0 & \lambda d & -\lambda d-1 & \cdots & 0 & 0 & 0 \\
\vdots & \vdots & \vdots & \ddots & \vdots & \vdots & \vdots \\
0 & 0 & 0 & \cdots & -\lambda d-1 & 1 & 0 \\
0 & 0 & 0 & \cdots & \lambda d & -\lambda d-1 & 1 \\
0 & 0 & 0 & \cdots & 0 & \lambda d & -\lambda d-1
\end{array}\right)
$$

with eigenvalue $-\mu<0$ or, equivalently, of the matrix

$$
M_{j}^{\prime}=M_{j}+(\lambda d+1) I_{j}=\left(\begin{array}{ccccccc}
0 & \lambda d & 0 & \cdots & 0 & 0 & 0 \\
1 & 0 & \lambda d & \cdots & 0 & 0 & 0 \\
0 & 1 & 0 & \cdots & 0 & 0 & 0 \\
\vdots & \vdots & \vdots & \ddots & \vdots & \vdots & \vdots \\
0 & 0 & 0 & \cdots & 0 & \lambda d & 0 \\
0 & 0 & 0 & \cdots & 1 & 0 & \lambda d \\
0 & 0 & 0 & \cdots & 0 & 1 & 0
\end{array}\right)
$$

with eigenvalue $\lambda d+1-\mu$. These matrices are tridiagonal Toeplitz matrices, and there is an exact formula for the eigenvalues and eigenvectors. (See, for instance, Example 7.2.5 in [16].) The Perron-Frobenius eigenvalue of $M_{j}^{\prime}$ is $2 \sqrt{\lambda d} \cos \left(\frac{\pi}{j+1}\right)$, with left eigenvector $\left(\gamma_{j, 1}, \ldots, \gamma_{j, j}\right)$ given by

$$
\gamma_{j, i}=(\lambda d)^{(j-i) / 2} \frac{\sin \left(\frac{i \pi}{j+1}\right)}{\sin \left(\frac{j \pi}{j+1}\right)} .
$$

This means that the largest eigenvalue of $M_{j}$ is $-\lambda d+O(\sqrt{\lambda d})$, so that we obtain

$$
(1+\lambda) \Delta Q_{j}(x) \simeq-\lambda d \frac{Q_{j}(x)}{n}+\frac{Q_{j+1}(x)}{n} \quad(1 \leq j<k),
$$

meaning that $Q_{j}(x)$ will drift to a value close to $Q_{j+1}(x) / \lambda d$. The choices of coefficients ensure that, if the $u_{j}(x)$ are all near to $\tilde{u}_{j} \simeq 1-(1-\lambda)(\lambda d)^{j-1}$, then

$$
Q_{j}(x)=n \sum_{i=1}^{j} \gamma_{j, i}\left(1-u_{i}(x)\right) \simeq n(1-\lambda) \sum_{i=1}^{j} \frac{\sin \left(\frac{i \pi}{j+1}\right)}{\sin \left(\frac{j \pi}{j+1}\right)}(\lambda d)^{i-1+(j-i) / 2},
$$

and the top term $i=j$ dominates the rest of the sum, provided $\lambda d$ is large, so $Q_{j}(x) \simeq$ $\left(1-u_{j}(x)\right)$ : this is also true for $j=k$. Thus the relationship $Q_{j} \simeq Q_{j+1} / \lambda d$ is as we would expect.

This means that, if $Q_{j+1}\left(X_{t}\right)$ remains in an interval around $\tilde{Q}_{j+1}:=n(1-\lambda)(\lambda d)^{j}$ for a long time, then $Q_{j}\left(X_{t}\right)$ will enter some interval around $\tilde{Q}_{j}$ within a short time, and stay there for a long time. We can then conduct the analysis for each $Q_{j}$ in turn, starting with $j=k$, to show that indeed all the $Q_{j}\left(X_{t}\right)$ quickly become close to $\tilde{Q}_{j}$, and stay close to $\tilde{Q}_{j}$ for a long time. This will then imply that the $u_{j}\left(X_{t}\right)$ all become and remain close to $\tilde{u}_{j}$.

A subsidiary application of this same technique forms another important step in the proofs (see the proof of Lemma 6.5(1)). If we do not assume that $u_{k+1}(x)$ is zero, but instead build this term into our calculations, we obtain the approximation 


$$
(1+\lambda) \Delta Q_{k}(x) \simeq\left(1-\lambda-u_{k+1}(x)\right)-\frac{Q_{k}(x)}{(\lambda d)^{k-1} n} .
$$

If $u_{k+1}\left(X_{t}\right)$ remains above $\varepsilon(1-\lambda)$, for some $\varepsilon>0$, for a long time, this drift equation tells us that $Q_{k}$ drifts down into an interval whose upper end is below the value $\tilde{Q}_{k}$, and then each of the $Q_{j}$ in turn drift down into intervals whose upper ends are below the corresponding $\tilde{Q}_{j}$, and remain there. For $j=1$, this means that the number of empty queues is at most $(1-\delta)(1-\lambda) n$, for some positive $\delta$, for a long period of time; this results in a persistent drift down in the total number of customers (since the departure rate is bounded below by $n-(1-\delta)(1-\lambda) n=\lambda n+\delta(1-\lambda) n$ while the arrival rate is $\lambda n)$, and this is not possible.

\section{Random Walks with Drifts}

In this section, we state some general results about the long-term behaviour of real-valued functions of a Markov chain with bounds on the drift. These are variants of results of Luczak and McDiarmid [11] and Brightwell and Luczak [2], and we do not give the proofs in full detail.

We start with a lemma concerning random walks with a drift, adapted from a result of Luczak and McDiarmid [11]. We have a sequence $\left(R_{t}\right)$ of real-valued random variables; on some "good" event, the jumps $Z_{t}=R_{t}-R_{t-1}$ have magnitude at most 1, and expectation at most $-v<0$. The lemma shows that, on the good event, with high probability, such a random walk, started at some value $r_{0}$, hits a lower value $r_{1}$ after not too many more than $\left(r_{0}-r_{1}\right) / v$ steps.

Lemma 3.1 Let $\varphi_{0} \subseteq \varphi_{1} \subseteq \cdots \subseteq \varphi_{m}$ be a filtration, and let $Z_{1}, \ldots, Z_{m}$ be random variables taking values in $[-1,1]$ such that each $Z_{i}$ is $\varphi_{i}$-measurable. Let $E_{0}, E_{1}, \ldots, E_{m-1}$ be events where $E_{i} \in \varphi_{i}$ for each $i$, and let $E=\bigcap_{i=0}^{m-1} E_{i}$. Fix $v \in(0,1)$, and let $r_{0}, r_{1} \in \mathbb{R}$ be such that $r_{0}>r_{1}$ and $v m \geq 2\left(r_{0}-r_{1}\right)$. Set $R_{0}=r_{0}$ and, for each integer $t>0$, let $R_{t}=R_{0}+\sum_{i=1}^{t} Z_{i}$.

Suppose that, for each $i=1, \ldots, m$,

$$
\mathbb{E}\left(Z_{i} \mid \varphi_{i-1}\right) \leq-v \text { on } E_{i-1} \cap\left\{R_{i-1}>r_{1}\right\} .
$$

Then

$$
\mathbb{P}\left(E \cap\left\{R_{t}>r_{1} \quad \forall t \in\{1, \ldots, m\}\right\}\right) \leq \exp \left(-\frac{v^{2} m}{8}\right) .
$$

We omit the proof, which is similar to one in [11].

For a discrete-time Markov process $\left(X_{t}\right)$ with state space $\mathcal{X}$, a real-valued function $F$ defined on $\mathcal{X}$, and an element $x$ of $\mathcal{X}$, we define

$$
\Delta F(x):=\mathbb{E}\left[F\left(X_{t+1}\right)-F\left(X_{t}\right) \mid X_{t}=x\right],
$$

and call this the drift of $F$ (at $x$ ). Similarly, we shall also use the notation $\Delta F\left(X_{t}\right)$ to denote the random variable $\mathbb{E}\left[F\left(X_{t+1}\right)-F\left(X_{t}\right) \mid X_{t}\right]$.

The next lemma says that, if the function $F$ has a negative drift of magnitude at least $v>0$ on a good set $\mathcal{U}$, and makes jumps of size at most 1 , then it is unlikely to increase by a large positive value before leaving $\mathcal{U}$.

Lemma 3.2 Let $a, v$ and $p$ be positive real numbers, with $v \leq p \leq 1$. Let $\left(X_{t}\right)_{t \geq 0}$ be $a$ discrete-time Markov process with state-space $\mathcal{X}$, adapted to the filtration $\left(\varphi_{t}\right)_{t \geq 0}$. Let $F$ be a 
real-valued function on $\mathcal{X}$ such that, $|F(y)-F(x)| \leq 1$ whenever $\mathbb{P}\left(X_{i+1}=y \mid X_{i}=x\right)>$ 0 . Let $\mathcal{U}$ be a subset of $\mathcal{X}$ such that, for $x \in \mathcal{U}, \Delta F(x) \leq-v$ and $\mathbb{P}\left(F\left(X_{i+1}\right)=F\left(X_{i}\right)\right.$ $\left.X_{i}=x\right) \geq 1-p$. Let $T_{\mathcal{U}}=\inf \left\{t: X_{t} \notin \mathcal{U}\right\}$, and let $T=\inf \left\{t: F\left(X_{t}\right)-F\left(X_{0}\right) \geq a\right\}$ Then

$$
\mathbb{P}\left(T \leq T_{\mathcal{U}}\right) \leq \frac{100}{v^{2}} e^{-v a / 4 p}
$$

Proof (Sketch) We use Theorem 2.5 of [2], applied to the function $F$. Translated into our setting, that result says that, for all $t \geq 0$, and all $\omega>0$,

$$
\mathbb{P}\left(\left\{T_{\mathcal{U}} \geq t\right\} \cap\left\{F\left(X_{t}\right)-F\left(X_{0}\right)+v t>\max (\sqrt{\omega p t}, \omega)\right\} \mid \varphi_{0}\right) \leq 2 e^{-\omega / 4} .
$$

For each $t$, we choose $\omega(t)=\frac{v}{2 p}(2 a+|v t-a|)$. It is easy to verify that $\max (\sqrt{\omega(t) p t}, \omega(t))<$ $v t+a$ for each $t$ (note that the hypotheses imply that $v \leq p$ ). Therefore

$$
\mathbb{P}\left(T<T_{\mathcal{U}} \mid \varphi_{0}\right) \leq 2 \sum_{t=0}^{\infty} e^{-\omega(t) / 4} \leq 4 e^{-v a / 4 p} \sum_{i=0}^{\infty} e^{-v^{2} i / 8 p} \leq \frac{100}{v^{2}} e^{-v a / 4 p},
$$

as desired.

We now use the two lemmas above to prove a result about real-valued functions of a Markov chain which we shall use repeatedly in our proofs.

Lemma 3.3 Let $h, v, c, \rho \geq 2, m, p \leq 1$ and s be positive real numbers with $v m \geq 2(c-h)$. Let $\left(X_{t}\right)_{t \geq 0}$ be a discrete-time Markov process with state-space $\mathcal{X}$, adapted to the filtration $\left(\varphi_{t}\right)_{t \geq 0}$. Let $\mathcal{S}$ be a subset of $\mathcal{X}$, and let $F$ be a real-valued function on $\mathcal{X}$ such that, for all $x \in \mathcal{S}$ with $F(x) \geq h$,

$$
\Delta F(x) \leq-v \text {, and } \mathbb{P}\left(F\left(X_{t+1}\right) \neq F\left(X_{t}\right) \mid X_{t}=x\right) \leq p,
$$

and for all $t \geq 0,\left|F\left(X_{t+1}\right)-F\left(X_{t}\right)\right| \leq 1$ a.s. Let $T^{*}$ be any stopping time, and suppose that $F\left(X_{T^{*}}\right) \leq c$ a.s.

Let

$$
\begin{aligned}
& T_{0}=\inf \left\{t \geq T^{*}: X_{t} \notin \mathcal{S}\right\}, \\
& T_{1}=\inf \left\{t \geq T^{*}: F\left(X_{t}\right) \leq h\right\}, \\
& T_{2}=\inf \left\{t>T_{1}: F\left(X_{t}\right) \geq h+\rho\right\} .
\end{aligned}
$$

Then

(i) $\mathbb{P}\left(T_{1} \wedge T_{0}>T^{*}+m\right) \leq \exp \left(-v^{2} m / 8\right)$;

(ii) $\mathbb{P}\left(T_{2} \leq s \wedge T_{0}\right) \leq \frac{100 s}{v^{2}} \exp (-\rho v / 8 p)$.

When we use the lemma, $m$ will be much smaller than $s$, with high probability $T^{*}$ will be much smaller than $s$, and also $\mathbb{P}\left(T_{0} \leq s\right)$ will be small. In these circumstances, the lemma allows us to conclude that $\mathbb{P}\left(T_{1}>T^{*}+m\right)$ and $\mathbb{P}\left(T_{2} \leq s\right)$ are small. This means that, with high probability, $F\left(X_{t}\right)$ decreases from its value at $T^{*}$ (at most $c$ ) to below $h$ in at most a further $m$ steps, and does not increase back above $h+\rho$ before time $s$. We shall sometimes use the conclusion of (ii) in the weaker form $\mathbb{P}\left(T_{2} \leq s<T_{0}\right) \leq \frac{100 s}{v^{2}} \exp (-\rho v / 8 p)$. For most uses of part (ii), we shall simply set $p=1$, but on occasion we need to use the stronger result in cases where the function $F$ rarely changes value. 
Proof We start by proving the lemma in the special case where the stopping time $T^{*}$ is equal to 0 .

For (i), we apply Lemma 3.1. The filtration $\varphi_{0} \subseteq \varphi_{1} \subseteq \cdots \subseteq \varphi_{m}$ will be the initial segment of the filtration $\left(\varphi_{t}\right)_{t \geq 0}$. For $t \geq 1$, we set $Z_{t}=F\left(X_{t}\right)-F\left(X_{t-1}\right)$, so that $R_{t}:=$ $R_{0}+\sum_{i=1}^{t} Z_{i}=F\left(X_{t}\right)$. For $t \geq 0$, we set $E_{t}$ to be the event that $T_{0}>t$ (i.e., $X_{i} \in \mathcal{S}$ for all $i$ with $0 \leq i \leq t$ ), so $E=\bigcap_{i=0}^{m-1} E_{i}$ is the event that $T_{0} \geq m$. We set $r_{0}=F\left(X_{0}\right) \leq c$, and $r_{1}=h$. We may assume that $r_{0}>r_{1}$; otherwise $T_{1}=0$ and there is nothing to prove.

On the event $E_{i-1} \cap\left\{R_{i-1}>r_{1}\right\}$, we have $X_{i-1} \in \mathcal{S}$ and $F\left(X_{i-1}\right)>r_{1}=h$, so $\mathbb{E}\left(Z_{i} \mid \varphi_{i-1}\right)=\Delta F\left(X_{i-1}\right) \leq-v$. Thus, noting that $v m \geq 2\left(r_{0}-r_{1}\right)$ by our assumption on $m$, we see that the conditions of Lemma 3.1 are satisfied. The event that $R_{t}>r_{1}$ for all $t=1, \ldots, m$ is the event that $T_{1}>m$, so

$$
\mathbb{P}\left(T_{1} \wedge T_{0}>m\right) \leq \mathbb{P}\left(\left\{T_{1}>m\right\} \cap\left\{T_{0} \geq m\right\}\right) \leq e^{-v^{2} m / 8},
$$

as required for (i).

We move on to (ii). For each time $r \in\{0, \ldots, s-1\}$, set

$$
T(r)=\min \left\{t \geq 0: F\left(X_{r+t}\right) \notin[h, h+\rho)\right\} .
$$

We say that $r$ is a departure point if: $T_{1} \leq r, F\left(X_{r}\right) \in[h, h+1), F\left(X_{r+T(r)}\right) \geq h+\rho$, and $r+T(r) \leq s \wedge T_{0}$. To say that $T_{2} \leq s \wedge T_{0}$ means that $F\left(X_{t}\right)$ crosses from its value, at most $h$, at time $T_{1}$, up to a value at least $h+\rho$, taking steps of size at most 1 , by time $s \wedge T_{0}$. This is equivalent to saying that there is at least one departure point $r \in[0, s)$. Therefore

$$
\begin{aligned}
\mathbb{P}\left(T_{2} \leq s \wedge T_{0}\right) \leq & \sum_{r=0}^{s-1} \mathbb{P}\left(\left\{T_{1} \leq r\right\} \cap\left\{F\left(X_{r}\right) \in[h, h+1)\right\}\right. \\
& \left.\cap\left\{F\left(X_{r+T(r)}\right) \geq h+\rho\right\} \cap\left\{r+T(r) \leq s \wedge T_{0}\right\}\right) \\
= & \sum_{r=0}^{s-1} \mathbb{E}\left[\mathbb{1}_{\left\{T_{1} \leq r\right\}} \mathbb{1}_{\left\{F\left(X_{r}\right) \in[h, h+1)\right\}} \mathbb{E}\left[\mathbb{1}_{\left\{F\left(X_{r+T(r)}\right) \geq h+\rho\right\}} \mathbb{1}_{\left\{r+T(r) \leq s \wedge T_{0}\right\}} \mid \varphi_{r}\right]\right] .
\end{aligned}
$$

Fix any $r \in[0, s)$. We claim that, for any $h_{0} \in[h, h+1)$, on the $\varphi_{r}$-measurable event that $F\left(X_{r}\right)=h_{0}$, the conditional expectation

$$
\mathbb{E}\left[\mathbb{1}_{\left\{F\left(X_{r+T(r)}\right) \geq h+\rho\right\}} \mathbb{1}_{\left\{r+T(r) \leq s \wedge T_{0}\right\}} \mid \varphi_{r}\right]
$$

is at most $\frac{100}{v^{2}} e^{-\rho v / 8 p}$. This will imply that each term of the sum above is at most $\frac{100}{v^{2}} e^{-\rho v / 8 p}$, and so that $\mathbb{P}\left(T_{2} \leq s \wedge T_{0}\right) \leq \frac{100 s}{v^{2}} \exp (-\rho v / 8 p)$, as required.

To prove the claim, we use Lemma 3.2. We consider the re-indexed process $\left(X_{t}^{\prime}\right)=\left(X_{r+t}\right)$; by the Markov property, this is a Markov chain with the same transition probabilities as $\left(X_{t}\right)$, and initial state $X_{0}^{\prime}=X_{r}$ with $F\left(X_{0}^{\prime}\right)=h_{0}$. We set $\varphi_{i}^{\prime}=\varphi_{r+i}$ for each $i$, so that $\left(X_{i}^{\prime}\right)$ is adapted to the filtration $\left(\varphi_{i}^{\prime}\right)$. We set $a=h+\rho-h_{0} \geq \rho-1 \geq \rho / 2$. We also set $\mathcal{U}=\mathcal{S} \cap\{x: F(x) \geq h\}, T_{\mathcal{U}}=\inf \left\{i: X_{i}^{\prime} \notin \mathcal{U}\right\}$, and $T=\inf \left\{i: F\left(X_{r+i}\right) \geq a\right\}$. Therefore, if $r+T(r) \leq T_{0}$ and $F\left(X_{r+T(r)}\right) \geq h+\rho$, then $T=T(r) \leq T_{\mathcal{U}}$.

For $i \leq T_{\mathcal{U}}$, we have $X_{i-1}^{\prime}=X_{r+i-1} \in \mathcal{S}$ and $F\left(X_{i-1}^{\prime}\right) \geq h$, and therefore $\Delta F\left(X_{i-1}^{\prime}\right) \leq$ $-v$, and also $\mathbb{P}\left(F\left(X_{i}^{\prime}\right)=F\left(X_{i-1}^{\prime}\right) \mid \varphi_{i-1}\right) \geq 1-p$. From Lemma 3.2, we now conclude that, on the event $F\left(X_{r}\right)=h_{0}$,

$$
\begin{aligned}
& \mathbb{P}\left(\left\{F\left(X_{r+T(r)}\right) \geq h+\rho\right\} \cap\left\{r+T(r) \leq s \leq T_{0}\right\} \mid \varphi_{r}\right) \\
& \quad \leq \mathbb{P}\left(T \leq T_{\mathcal{U}}\right) \leq \frac{100}{v^{2}} e^{-v a / 4 p} \leq \frac{100}{v^{2}} e^{-\rho v / 8 p},
\end{aligned}
$$


as required. This completes the proof in the special case where $T^{*}=0$.

We now proceed to the general case. Suppose then that the hypotheses of the lemma are satisfied, with stopping time $T^{*}$. We apply the result we have just proved to the process $\left(X_{t}^{\prime}\right)=\left(X_{T^{*}+t}\right)$. By the strong Markov property, $\left(X_{t}^{\prime}\right)$ is also a Markov process, adapted to the filtration $\left(\varphi_{t}^{\prime}\right)_{t \geq 0}=\left(\varphi_{T^{*}+t}\right)_{t \geq 0}$. The condition that $F\left(X_{T^{*}}\right) \leq c$ is equivalent to $F\left(X_{0}^{\prime}\right) \leq c$. Set:

$$
\begin{aligned}
& T_{0}^{\prime}=\inf \left\{t \geq 0: X_{t}^{\prime} \notin \mathcal{S}\right\}=\inf \left\{t \geq 0: X_{T^{*}+t} \notin \mathcal{S}\right\}=T_{0}-T^{*} \\
& T_{1}^{\prime}=\inf \left\{t \geq 0: F\left(X_{t}^{\prime}\right) \leq h\right\}=\inf \left\{t \geq 0: F\left(X_{T^{*}+t}\right) \leq h\right\}=T_{1}-T^{*} \\
& T_{2}^{\prime}=\inf \left\{t>T_{1}^{\prime}: F\left(X_{t}^{\prime}\right) \geq h+\rho\right\}=\inf \left\{t>T_{1}^{\prime}: F\left(X_{T^{*}+t}\right) \geq h+\rho\right\}=T_{2}-T^{*},
\end{aligned}
$$

and note that these are all stopping times with respect to the filtration $\left(\varphi_{t}^{\prime}\right)$. The special case of the result (with $T^{*}=0$ ) now tells us that:

$$
\text { (i) } \begin{aligned}
\mathbb{P}\left(T_{1} \wedge T_{0}>T^{*}+m\right) & =\mathbb{P}\left(\left(T^{*}+T_{1}^{\prime}\right) \wedge\left(T^{*}+T_{0}^{\prime}\right)>T^{*}+m\right) \\
& =\mathbb{P}\left(T_{1}^{\prime} \wedge T_{0}^{\prime}>m\right) \\
& \leq \exp \left(-v^{2} m / 8\right) ; \\
\text { (ii) } \quad \mathbb{P}\left(T_{2} \leq s \wedge T_{0}\right) & =\mathbb{P}\left(T^{*}+T_{2}^{\prime} \leq s \wedge\left(T^{*}+T_{0}^{\prime}\right)\right) \\
& \leq \mathbb{P}\left(T^{*}+T_{2}^{\prime} \leq\left(T^{*}+s\right) \wedge\left(T^{*}+T_{0}^{\prime}\right)\right) \\
& =\mathbb{P}\left(T_{2}^{\prime} \leq s \wedge T_{0}^{\prime}\right) \\
& \leq \frac{100 p}{v^{2}} \exp (-\rho v / 8 p) .
\end{aligned}
$$

In both cases, these are the desired results.

We also use a "reversed" version of Lemma 3.3 where $\Delta F(x) \geq v$ for all $x$ in some "good" set $\mathcal{S}$ with $F(x) \leq h$. The result and proof are practically identical to Lemma 3.3, changing the directions of inequalities where necessary, and using "reversed" versions of Lemmas 3.1 and 3.2.

The next lemma is a more precise version of Lemma 2.2 in [11]. We omit the proof, which is exactly as in [11], except that we track more carefully the values of the various constants appearing in that proof, and separate out the effects of the two occurrences of $\delta$ in that theorem. We will use this result in our proof of Lemma 10.1, showing rapid mixing.

Lemma 3.4 Let $\left(\varphi_{t}\right)_{t \geq 0}$ be a filtration. Let $Z_{1}, Z_{2}, \ldots$ be $\{0, \pm 1\}$-valued random variables, where each $Z_{i}$ is $\varphi_{i}$-measurable. Let $S_{0} \geq 0$ a.s., and for each positive integer $j$ let $S_{j}=$ $S_{0}+\sum_{i=1}^{j} Z_{i}$. Let $A_{0}, A_{1}, \ldots$ be events, where each $A_{i}$ is $\varphi_{i}$-measurable.

Suppose that there is a positive integer $k_{0}$ and a constant $\delta$ with $0<\delta<1 / 2$ such that $\mathbb{P}\left(Z_{i}=-1 \mid \varphi_{i-1}\right) \geq \delta$ on $A_{i-1} \cap\left\{S_{i-1} \in\left\{1, \ldots, k_{0}-1\right\}\right\}$ and $\mathbb{P}\left(Z_{i}=-1 \mid \varphi_{i-1}\right) \geq 3 / 4$ on $A_{i-1} \cap\left\{S_{i-1} \geq k_{0}\right\}$. Then, for each positive integer $m$

$$
\mathbb{P}\left(\bigcap_{i=1}^{m}\left\{S_{i} \neq 0\right\} \cap \bigcap_{i=0}^{m-1} A_{i}\right) \leq \mathbb{P}\left(S_{0}>\lfloor m / 16\rfloor\right)+3 \exp \left(-\frac{\delta^{k_{0}-1}}{200 k_{0}} m\right) .
$$

Several times we shall use the fact that, if $Z$ is a binomial or Poisson random variable with mean $\mu$, then for each $0 \leq \epsilon \leq 1$ we have

$$
\mathbb{P}(Z-\mu \leq-\epsilon \mu) \leq e^{-(1 / 2) \epsilon^{2} \mu} .
$$




\section{Coupling}

We now introduce a natural coupling of copies of the $(n, d, \lambda)$-supermarket process $\left(X_{t}^{x}\right)$ with different initial states $x$. The coupling is a natural adaptation to discrete time of that in [11]. In this section, we make no assumptions about the values of the parameters $n, \lambda$ and $d$.

We describe the coupling in terms of three independent sequences of random variables. There is an iid sequence $\mathbf{V}=\left(V_{1}, V_{2}, \ldots\right)$ of $0-1$ random variables where each $V_{i}$ takes value 1 with probability $\lambda /(1+\lambda) ; V_{i}=1$ if and only if time $i$ is an arrival. Corresponding to every time $i$ there is also an ordered list $D_{i}$ of $d$ queue indices, each chosen uniformly at random with replacement. Let $\mathbf{D}=\left(D_{1}, D_{2}, \ldots\right)$. Furthermore, corresponding to every time $i$ there is a uniformly chosen queue index $\tilde{D}_{i}$. Let $\tilde{\mathbf{D}}=\left(\tilde{D}_{1}, \tilde{D}_{2}, \ldots\right)$. At time $i, D_{i}$ will be used if $Z_{i}=1$, and there will be an arrival to the first shortest queue in $D_{i}$; otherwise, there will be a departure from the queue with index $\tilde{D}_{i}$, if that queue is currently non-empty.

Suppose that we are given a realisation $(\mathbf{v}, \mathbf{d}, \tilde{\mathbf{d}})$ of $(\mathbf{V}, \mathbf{D}, \tilde{\mathbf{D}})$. For each possible initial queue-lengths vector $x \in \mathbb{Z}_{+}^{n}$, this realisation yields a deterministic process $\left(x_{t}\right)$ with $x_{0}=x$ : let us write $x_{t}=s_{t}(x ; \mathbf{v}, \mathbf{d}, \tilde{\mathbf{d}})$. Then, for each $x \in \mathbb{Z}_{+}^{n}$, the process $s_{t}(x ; \mathbf{V}, \mathbf{D}, \tilde{\mathbf{D}})$ has the distribution of the $(n, d, \lambda)$-supermarket process $X_{t}^{x}$ with initial state $x$. In this way, we construct copies $\left(X_{t}^{x}\right)$ of the $(n, d, \lambda)$-supermarket process for each possible starting state $x$ on a single probability space. When we treat more than one such copy at the same time, we always work in this probability space, and we let $\mathbb{P}(\cdot)$ denote the corresponding coupling measure.

We shall use the following lemma, which is a discrete-time analogue of Lemma 2.3 in [11] and is proved in exactly the same way.

Lemma 4.1 Fix any triple $\mathbf{z}, \mathbf{d}, \tilde{\mathbf{d}}$ as above, and for each queue-lengths vector $x$, write $s_{t}(x)$ for $s_{t}(x ; \mathbf{z}, \mathbf{d}, \tilde{\mathbf{d}})$. Then, for each $x, y \in \mathbb{Z}_{+}^{n}$, both $\left\|s_{t}(x)-s_{t}(y)\right\|_{1}$ and $\left\|s_{t}(x)-s_{t}(y)\right\|_{\infty}$ are nonincreasing; and further, if $0 \leq t<t^{\prime}$ and $s_{t}(x) \leq s_{t}(y)$, then $s_{t^{\prime}}(x) \leq s_{t^{\prime}}(y)$.

Given positive real numbers $\ell$ and $b$, we set

$$
\begin{aligned}
& \mathcal{A}_{0}(\ell, b)=\left\{x:\|x\|_{\infty} \leq \ell \text { and }\|x\|_{1} \leq b n\right\} \\
& \mathcal{A}_{1}(\ell, b)=\left\{x:\|x\|_{\infty} \leq 3 \ell \text { and }\|x\|_{1} \leq 3 b n\right\} .
\end{aligned}
$$

We also set

$$
\ell^{*}=(1-\lambda)^{-1} \log ^{2} n, b^{*}=2(1-\lambda)^{-1}, \mathcal{A}_{0}=\mathcal{A}_{0}\left(\ell^{*}, b^{*}\right), \mathcal{A}_{1}=\mathcal{A}_{1}\left(\ell^{*}, b^{*}\right) .
$$

Thus a state $x$ is in $\mathcal{A}_{0}$ if there are at most $2 n(1-\lambda)^{-1}$ customers in total, and no more than $(1-\lambda)^{-1} \log ^{2} n$ in any queue. These requirements are relaxed by a factor of 3 in $\mathcal{A}_{1}$.

The next result tells us that the $(n, d, \lambda)$-supermarket process $\left(Y_{t}\right)$, in equilibrium, is very unlikely to be outside the set $\mathcal{A}_{0}$, for any $d$. This is accomplished by proving the result for $d=1$, when the process is easy to analyse explicitly, and then using coupling in $d$ to deduce the result for all $d$. Of course, the result is actually extremely weak for all $d>1$, and later we shall show a much stronger result whenever the various parameters of the model satisfy the conditions of Theorem 1.1; the importance of the lemma below is that it gets us started and enables us to say something about where the equilibrium of the process lives.

Lemma 4.2 Let $\left(Y_{t}\right)$ be a copy of the $(n, d, \lambda)$-supermarket process in equilibrium. Then $\mathbb{P}\left(Y_{t} \notin \mathcal{A}_{0}\right) \leq 2 n e^{-\log ^{2} n}$. 
Proof Let $\tilde{Y}$ denote a stationary copy of the $(n, 1, \lambda)$-supermarket process, in which each arriving customer joins a uniform random queue. Then the queue lengths $\tilde{Y}_{t}(j)$ are independent geometric random variables with mean $\lambda /(1-\lambda)$, where $\mathbb{P}\left(\tilde{Y}_{t}(j)=r\right)=(1-\lambda) \lambda^{r}$ for $r=0,1,2, \ldots$. Therefore, $\mathbb{P}\left(\left\|\tilde{Y}_{t}\right\|_{\infty} \geq r\right) \leq n \lambda^{r}$, and also it can easily be checked that $\mathbb{P}\left(\left\|\tilde{Y}_{t}\right\|_{1} \geq 2 n(1-\lambda)^{-1}\right) \leq e^{-n / 4}$.

As mentioned in the remarks after Theorem 1.1, there is a coupling between supermarket processes with different values of $d$, which can be used to show that the equilibrium copy $\left(Y_{t}\right)$ of the $(n, d, \lambda)$-supermarket process, for any $d$, also satisfies $\mathbb{P}\left(\left\|Y_{t}\right\|_{1} \geq 2 n(1-\lambda)^{-1}\right) \leq$ $e^{-n / 4}$ and $\mathbb{P}\left(\left\|Y_{t}\right\|_{\infty} \geq \log ^{2} n(1-\lambda)^{-1}\right) \leq n \lambda^{\log ^{2} n(1-\lambda)^{-1}} \leq n e^{-\log ^{2} n}$, as required.

Next we prove a very crude concentration of measure result: if the process $\left(Y_{t}\right)$ in equilibrium is concentrated inside some set $\mathcal{A}_{0}(\ell, b)$, and we start a copy $\left(X_{t}^{x}\right)$ of the process at a state $x \in \mathcal{A}_{0}(\ell, b)$, then the process $\left(X_{t}^{x}\right)$ is unlikely to leave the larger set $\mathcal{A}_{1}(\ell, b)$ over a long period of time.

Lemma 4.3 Let $\ell$ and $b$ be natural numbers and $x$ a queue-lengths vector in $\mathcal{A}_{0}(\ell, b)$. Let $\left(Y_{t}\right)$ be a copy of the $(n, d, \lambda)$-supermarket process in equilibrium, and let $\left(X_{t}^{x}\right)$ be a copy started in state $x$. Then for any natural number $s$,

$$
\mathbb{P}\left(\exists t \in[0, s], X_{t}^{x} \notin \mathcal{A}_{1}(\ell, b)\right) \leq \mathbb{P}\left(\exists t \in[0, s], Y_{t} \notin \mathcal{A}_{0}(\ell, b)\right) .
$$

Proof By Lemma 4.1, we can couple $\left(X_{t}^{x}\right)$ and $\left(Y_{t}\right)$ in such a way that $\left\|X_{t}^{x}-Y_{t}\right\|_{1}$ and $\left\|X_{t}^{x}-Y_{t}\right\|_{\infty}$ are both non-increasing, and hence that, for each $t \geq 0$,

$$
\begin{aligned}
\left\|X_{t}^{x}\right\|_{1} & \leq\left\|X_{t}^{x}-Y_{t}\right\|_{1}+\left\|Y_{t}\right\|_{1} \leq\left\|x-Y_{0}\right\|_{1}+\left\|Y_{t}\right\|_{1} \\
& \leq\|x\|_{1}+\left\|Y_{0}\right\|_{1}+\left\|Y_{t}\right\|_{1} \leq b n+\left\|Y_{0}\right\|_{1}+\left\|Y_{t}\right\|_{1},
\end{aligned}
$$

and similarly

$$
\left\|X_{t}^{x}\right\|_{\infty} \leq \ell+\left\|Y_{0}\right\|_{\infty}+\left\|Y_{t}\right\|_{\infty}
$$

We deduce that, for each $t \geq 0$,

$$
\begin{aligned}
\left\{X_{t}^{x} \notin \mathcal{A}_{1}(\ell, b)\right\} & =\left\{\left\|X_{t}^{x}\right\|_{1}>3 b n\right\} \cup\left\{\left\|X_{t}^{x}\right\|_{\infty}>3 \ell\right\} \\
& \subseteq\left\{\left\|Y_{0}\right\|_{1}>b n\right\} \cup\left\{\left\|Y_{t}\right\|_{1}>b n\right\} \cup\left\{\left\|Y_{0}\right\|_{\infty}>\ell\right\} \cup\left\{\left\|Y_{t}\right\|_{\infty}>\ell\right\} \\
& =\left\{Y_{0} \notin \mathcal{A}_{0}(\ell, b)\right\} \cup\left\{Y_{t} \notin \mathcal{A}_{0}(\ell, b)\right\} .
\end{aligned}
$$

The result now follows immediately.

We shall use Lemma 4.3 later for general values of $\ell$ and $b$, but for now we note the following immediate consequence of the previous two lemmas. Let $T_{\mathcal{A}}^{\dagger}=T_{\mathcal{A}}^{\dagger}(x)=\inf \{t$ : $\left.X_{t}^{x} \notin \mathcal{A}_{1}\right\}$ : this will be an instance of a more general notation we introduce later: when we have a pair of sets $\mathcal{S}_{0} \subseteq \mathcal{S}_{1}$, we will use $T_{\mathcal{S}}$ to denote the first time we enter the inner set, and $T_{\mathcal{S}}^{\dagger}$ to denote the first time after $T_{\mathcal{S}}$ that we leave the outer one.

Lemma 4.4 Let $x$ be any queue-lengths vector in $\mathcal{A}_{0}$. Then, for $n$ sufficiently large,

$$
\mathbb{P}\left(T_{\mathcal{A}}^{\dagger}(x) \leq e^{\frac{1}{3} \log ^{2} n}\right) \leq e^{-\frac{1}{2} \log ^{2} n} .
$$

Proof The probability in question is $\mathbb{P}\left(\exists t \in\left[0, e^{\frac{1}{3} \log ^{2} n}\right], X_{t}^{x} \notin \mathcal{A}_{1}\right)$ which, by Lemma 4.3 and Lemma 4.2, is at most

$$
\mathbb{P}\left(\exists t \in\left[0, e^{\frac{1}{3} \log ^{2} n}\right], Y_{t} \notin \mathcal{A}_{0}\right) \leq\left(e^{\frac{1}{3} \log ^{2} n}+1\right) \mathbb{P}\left(Y_{t} \notin \mathcal{A}_{0}^{*}\right) \leq 3 n e^{-\frac{2}{3} \log ^{2} n},
$$

which, for $n$ sufficiently large, is at most $e^{-\frac{1}{2} \log ^{2} n}$, as required. 


\section{Functions and Drifts}

We now start the detailed proofs of our main results.

As explained in Sect. 2, we will consider a sequence of functions $Q_{k}, Q_{k-1}, \ldots, Q_{1}$ defined on the set $\mathbb{Z}_{+}^{n}$ of queue-lengths vectors. We now give precise definitions of these functions, along with another function $P_{k-1}$, and derive some of their properties.

The results in this section will be used in the course of the proof of Theorem 1.1, and we could assume that we are in the regime covered by our theorem; however, for this section all that is necessary is that $\lambda d \geq 16$. In the special case $k=1$, we need only consider the function $Q_{k}=Q_{1}$ and its drift; otherwise we assume that $k \geq 2$.

As in Sect. 2, let $Q_{k}$ be the function defined on the set $\mathbb{Z}_{+}^{n}$ of all queue-lengths vectors by

$$
Q_{k}(x)=n \sum_{i=1}^{k} \gamma_{i}\left(1-u_{i}(x)\right)
$$

where, for $i=1, \ldots, k$,

$$
\gamma_{i}=1-\frac{1}{(\lambda d)^{i}}-\frac{i-1}{(\lambda d)^{k}}
$$

It is also convenient to set $\gamma_{0}=0$. Evidently $\gamma_{i}<1$ for each $i$, an inequality we shall use freely in future. We also note that, provided $\lambda d>2$,

$$
\gamma_{i+1}-\gamma_{i}=\frac{1}{(\lambda d)^{i}}-\frac{1}{(\lambda d)^{i+1}}-\frac{1}{(\lambda d)^{k}},
$$

for $i=0, \ldots, k-1$. Therefore $\gamma_{i}$ is increasing in $i$; also $\gamma_{k}=1-k(\lambda d)^{-k}$.

If $k \geq 2$, we set $P_{k-1}(x)=n \sum_{i=1}^{k-1}\left(1-u_{i}(x)\right)$. Also, for $j=1, \ldots, k-1$, we let $Q_{j}(x)=n \sum_{i=1}^{j} \gamma_{j, i}\left(1-u_{i}(x)\right)$, where the coefficients $\gamma_{j, i}$ are given by

$$
\gamma_{j, i}=(\lambda d)^{(j-i) / 2} \frac{\sin \left(\frac{i \pi}{j+1}\right)}{\sin \left(\frac{j \pi}{j+1}\right)} .
$$

Consistent with the expression above, we also define $\gamma_{j, 0}=\gamma_{j, j+1}=0$. It can easily be checked that, for each $i=1, \ldots, j-1$, and for each $j=1, \ldots, k-1$,

$$
\lambda d \gamma_{j, i+1}+\gamma_{j, i-1}=2 \sqrt{\lambda d} \cos \left(\frac{\pi}{j+1}\right) \gamma_{j, i} .
$$

This is equivalent to saying that the $\gamma_{j, i}$ form eigenvectors of the tridiagonal Toeplitz matrices $M_{j}$ given in Sect. 2, with eigenvalue $-\lambda d-1+2 \sqrt{\lambda d} \cos \left(\frac{\pi}{j+1}\right)$.

We will need some bounds on the sizes of the $Q_{j}(x)$, for $j<k$. Observe that $\gamma_{j, j}=1$ for each $j$, while generally we have

$$
1 \leq \frac{\sin (i \pi /(j+1))}{\sin (j \pi /(j+1))}=\frac{\sin (i \pi /(j+1))}{\sin (\pi /(j+1))} \leq i,
$$

since the sine function is concave on $[0, \pi]$. Thus $(\lambda d)^{(j-i) / 2} \leq \gamma_{j, i} \leq i(\lambda d)^{(j-i) / 2}$ and therefore

$$
Q_{j}(x) \leq n \sum_{i=1}^{j} i(\lambda d)^{(j-i) / 2} \leq \frac{n}{(1-1 / \sqrt{\lambda d})^{2}} \leq 2 n(\lambda d)^{(j-1) / 2},
$$


provided $\lambda d \geq 16$. We also note at this point that changing one component $x(\ell)$ of $x$ by \pm 1 changes $Q_{j}(x)$ by at most $\gamma_{j, 1}=(\lambda d)^{(j-1) / 2}$.

It can readily be checked that, for $j \geq 1$, the function

$$
f(i)=\sin \left(\frac{i \pi}{j+2}\right) / \sin \left(\frac{i \pi}{j+1}\right)
$$

is increasing over the range $[1, j]$, and so we have, for $1 \leq i \leq j \leq k-2$ :

$$
\begin{aligned}
\frac{\gamma_{j+1, i}}{\gamma_{j, i}} & =\sqrt{\lambda d} \frac{\sin (i \pi /(j+2)) \sin (\pi /(j+1))}{\sin (i \pi /(j+1)) \sin (\pi /(j+2))} \\
& \leq \sqrt{\lambda d} \frac{\sin (j \pi /(j+2)) \sin (\pi /(j+1))}{\sin (j \pi /(j+1)) \sin (\pi /(j+2))} \\
& =\sqrt{\lambda d} \frac{\sin (2 \pi /(j+2))}{\sin (\pi /(j+2))} \leq 2 \sqrt{\lambda d},
\end{aligned}
$$

using (5.2) for the final inequality. A consequence is that, for $j=1, \ldots, k-2$, and any $x \in \mathbb{Z}_{+}^{n}$,

$$
\begin{aligned}
\frac{Q_{j+1}(x)}{n} & =\left(1-u_{j+1}(x)\right)+\sum_{i=1}^{j} \gamma_{j+1, i}\left(1-u_{i}(x)\right) \\
& \leq\left(1-u_{j+1}(x)\right)+\sum_{i=1}^{j} 2 \sqrt{\lambda d} \gamma_{j, i}\left(1-u_{i}(x)\right) \\
& \leq\left(1-u_{j+1}(x)\right)+2 \sqrt{\lambda d} \frac{Q_{j}(x)}{n} .
\end{aligned}
$$

For $j=k-1$, we have the stronger inequality that, for any $x \in \mathbb{Z}_{+}^{n}$,

$$
\frac{Q_{k}(x)}{n} \leq \sum_{i=1}^{k}\left(1-u_{i}(x)\right) \leq\left(1-u_{k}(x)\right)+\frac{Q_{k-1}(x)}{n} .
$$

We now prove that the drift of the function $Q_{k}(x)$ is approximately equal to $\frac{1}{1+\lambda}\left(1-\lambda-u_{k+1}(x)-\frac{1}{(\lambda d)^{k-1}} \frac{Q_{k}(x)}{n}\right)$.

Lemma 5.1 If $k \geq 2$, then, for any state $x \in \mathbb{Z}_{+}^{n}$,

$$
\begin{aligned}
(1+\lambda) \Delta Q_{k}(x) \leq & \gamma_{k}\left((1-\lambda)-u_{k+1}(x)+\lambda \exp \left(-d Q_{k}(x) / k n\right)\right) \\
& -\frac{1}{(\lambda d)^{k-1}} \frac{Q_{k}(x)}{n}\left(1-\frac{2}{\lambda d}\right), \\
(1+\lambda) \Delta Q_{k}(x) \geq & \gamma_{k}\left((1-\lambda)-u_{k+1}(x)\right)-\frac{1}{(\lambda d)^{k-1}} \frac{Q_{k}(x)}{n} \\
& -\left(\frac{Q_{k-1}(x)}{n}\right)^{2} \frac{1}{(\lambda d)^{k-3}} .
\end{aligned}
$$

For $k=1$, we have

$$
\begin{aligned}
& (1+\lambda) \Delta Q_{1}(x) \leq \gamma_{1}\left((1-\lambda)-u_{2}(x)+\lambda \exp \left(-d Q_{1}(x) / n\right)\right)-\frac{Q_{1}(x)}{n}, \\
& (1+\lambda) \Delta Q_{1}(x) \geq \gamma_{1}\left((1-\lambda)-u_{2}(x)\right)-\frac{Q_{1}(x)}{n} .
\end{aligned}
$$


Proof As in (2.1), we have that, for $i=1, \ldots, k$,

$$
\Delta u_{i}(x)=\frac{1}{n(1+\lambda)}\left(\lambda u_{i-1}(x)^{d}-\lambda u_{i}(x)^{d}-u_{i}(x)+u_{i+1}(x)\right) .
$$

and that $u_{0}$ is identically equal to 1 . We deduce that

$$
\begin{aligned}
\Delta Q_{k}(x) & =-n \sum_{i=1}^{k} \gamma_{i} \Delta u_{i}(x) \\
& =\frac{1}{1+\lambda} \sum_{i=1}^{k} \gamma_{i}\left(-\lambda u_{i-1}(x)^{d}+\lambda u_{i}(x)^{d}+u_{i}(x)-u_{i+1}(x)\right) .
\end{aligned}
$$

We rearrange the formula above as follows:

$$
\begin{aligned}
(1+\lambda) \Delta Q_{k}(x)= & \gamma_{k}\left((1-\lambda)+\lambda u_{k}(x)^{d}-u_{k+1}(x)+\lambda\left(1-u_{k-1}(x)^{d}\right)-\left(1-u_{k}(x)\right)\right) \\
& +\sum_{i=1}^{k-1} \gamma_{i}\left(\lambda\left(1-u_{i-1}(x)^{d}\right)-\lambda\left(1-u_{i}(x)^{d}\right)\right. \\
& \left.-\left(1-u_{i}(x)\right)+\left(1-u_{i+1}(x)\right)\right) \\
= & \gamma_{k}\left((1-\lambda)+\lambda u_{k}(x)^{d}-u_{k+1}(x)\right) \\
& +\lambda \sum_{i=1}^{k-1}\left(\gamma_{i+1}-\gamma_{i}\right)\left(1-u_{i}(x)^{d}\right)-\sum_{i=1}^{k}\left(\gamma_{i}-\gamma_{i-1}\right)\left(1-u_{i}(x)\right) .
\end{aligned}
$$

Here we have used the facts that $\gamma_{0}=0$ and $1-u_{0}(x)=0$.

Now, for $1 \leq i \leq k$, we have $1-u_{i}(x) \leq 1-u_{k}(x)$ for all $x$, and $\gamma_{i} \leq 1$. Therefore $Q_{k}(x) \leq n k\left(1-u_{k}(x)\right)$, and hence

$$
0 \leq u_{k}(x)^{d} \leq\left(1-\frac{Q_{k}(x)}{k n}\right)^{d} \leq \exp \left(-d Q_{k}(x) / k n\right) .
$$

For $k \geq 2$, in order to estimate the terms constituting the two sums, we note the inequalities $d(1-u)-\left(\begin{array}{c}d \\ 2\end{array}\right)(1-u)^{2} \leq 1-u^{d} \leq d(1-u)$. To obtain our upper bound on $\Delta Q_{k}(x)$, we apply the inequality $1-u_{i}(x)^{d} \leq d\left(1-u_{i}(x)\right)$ for each $i=1, \ldots, k-1$. Using also (5.1), we have

$$
\begin{aligned}
\lambda \sum_{i=1}^{k-1}\left(\gamma_{i+1}-\gamma_{i}\right)\left(1-u_{i}(x)^{d}\right)-\sum_{i=1}^{k}\left(\gamma_{i}-\gamma_{i-1}\right)\left(1-u_{i}(x)\right) \\
\leq \lambda d \sum_{i=1}^{k-1}\left(\gamma_{i+1}-\gamma_{i}\right)\left(1-u_{i}(x)\right)-\sum_{i=1}^{k}\left(\gamma_{i}-\gamma_{i-1}\right)\left(1-u_{i}(x)\right) \\
=-\left[\frac{1}{(\lambda d)^{k-1}}-\frac{2}{(\lambda d)^{k}}\right]\left(1-u_{k}(x)\right) \\
\quad+\sum_{i=1}^{k-1}\left[\frac{\lambda d}{(\lambda d)^{i}}-\frac{\lambda d}{(\lambda d)^{i+1}}-\frac{\lambda d}{(\lambda d)^{k}}-\frac{1}{(\lambda d)^{i-1}}+\frac{1}{(\lambda d)^{i}}+\frac{1}{(\lambda d)^{k}}\right]\left(1-u_{i}(x)\right)
\end{aligned}
$$




$$
\begin{aligned}
& =-\frac{1}{(\lambda d)^{k-1}}\left[\left(1-\frac{2}{\lambda d}\right)\left(1-u_{k}(x)\right)+\sum_{i=1}^{k-1}\left(1-\frac{1}{\lambda d}\right)\left(1-u_{i}(x)\right)\right] \\
& \leq-\frac{1}{(\lambda d)^{k-1}} \frac{Q_{k}(x)}{n}\left(1-\frac{2}{\lambda d}\right) .
\end{aligned}
$$

This establishes the required upper bound on $(1+\lambda) \Delta Q_{k}(x)$. The calculation works because the $\gamma_{i}$ are the entries of a good approximation to the Perron-Frobenius eigenvector of the matrix $M_{k}$ defined in Sect. 2.

For the lower bound, the previous calculation, and the bound $1-u_{i}(x)^{d} \geq d(1-u)-$ $\left(\begin{array}{l}d \\ 2\end{array}\right)(1-u)^{2}$, lead us to

$$
\begin{aligned}
& \lambda \sum_{i=1}^{k-1}\left(\gamma_{i+1}-\gamma_{i}\right)\left(1-u_{i}(x)^{d}\right)-\sum_{i=1}^{k}\left(\gamma_{i}-\gamma_{i-1}\right)\left(1-u_{i}(x)\right) \\
& \geq-\lambda\left(\begin{array}{l}
d \\
2
\end{array}\right) \sum_{i=1}^{k-1}\left(\gamma_{i+1}-\gamma_{i}\right)\left(1-u_{i}(x)\right)^{2} \\
& \quad-\frac{1}{(\lambda d)^{k-1}}\left[\left(1-\frac{2}{\lambda d}\right)\left(1-u_{k}(x)\right)+\sum_{i=1}^{k-1}\left(1-\frac{1}{\lambda d}\right)\left(1-u_{i}(x)\right)\right] \\
& \geq-\lambda\left(\begin{array}{l}
d \\
2
\end{array}\right) \sum_{i=1}^{k-1}\left(\gamma_{i+1}-\gamma_{i}\right)\left(1-u_{i}(x)\right)^{2}-\frac{1}{(\lambda d)^{k-1}} \frac{Q_{k}(x)}{n} .
\end{aligned}
$$

Here we used the fact that $1-1 /(\lambda d) \leq \gamma_{i}$ for each $i$.

It remains to show that

$$
\lambda\left(\begin{array}{l}
d \\
2
\end{array}\right) \sum_{i=1}^{k-1}\left(\gamma_{i+1}-\gamma_{i}\right)\left(1-u_{i}(x)\right)^{2} \leq\left(\frac{Q_{k-1}(x)}{n}\right)^{2} \frac{1}{(\lambda d)^{k-3}} .
$$

We observe that

$$
\begin{aligned}
\left(\frac{Q_{k-1}(x)}{n}\right)^{2} & =\left(\sum_{i=1}^{k-1}(\lambda d)^{(k-1-i) / 2} \frac{\sin \left(\frac{i \pi}{k}\right)}{\sin \left(\frac{(k-1) \pi}{k}\right)}\left(1-u_{i}(x)\right)\right)^{2} \\
& \geq \sum_{i=1}^{k-1}(\lambda d)^{k-1-i}\left(1-u_{i}(x)\right)^{2} \\
& \geq(\lambda d)^{k-1} \sum_{i=1}^{k-1}\left(\gamma_{i+1}-\gamma_{i}\right)\left(1-u_{i}(x)\right)^{2}
\end{aligned}
$$

which implies the required inequality.

In the special case $k=1$, the equation for the drift reduces to

$$
(1+\lambda) \Delta Q_{1}(x)=\gamma_{1}\left(1-\lambda-u_{2}(x)\right)-\frac{Q_{1}(x)}{n}+\gamma_{1} \lambda u_{1}(x)^{d},
$$

and both the required bounds follow immediately.

We prove a similar result for the functions $Q_{j}(x), 1 \leq j \leq k-1$. Ideally, the drift bounds would be expressed in terms of $Q_{j}(x)$ itself and $Q_{j+1}(x)$ : however, there is a complication. 
In the upper bound, there appears a term which can be bounded above by $\lambda\left(\begin{array}{l}d \\ 2\end{array}\right) \sum_{i=1}^{j} \gamma_{j, i}(1-$ $\left.u_{i}(x)\right)^{2}$, and we would like to show that this is small compared with $\lambda d \sum_{i=1}^{j} \gamma_{j, i}\left(1-u_{i}(x)\right)$. This is true if $1-u_{j}(x) \ll 1 / d$, but in general we cannot assume this. We bound this term above, very crudely, by

$$
\lambda\left(\begin{array}{l}
d \\
2
\end{array}\right)\left(\sum_{i=1}^{k-1}\left(1-u_{i}(x)\right)\right)\left(\sum_{i=1}^{j} \gamma_{j, i}\left(1-u_{i}(x)\right)\right)=\lambda\left(\begin{array}{l}
d \\
2
\end{array}\right) \frac{P_{k-1}(x) Q_{j}(x)}{n^{2}} ;
$$

we use the function $P_{k-1}$ here because its drifts are relatively easy to handle.

Lemma 5.2 Fix $j$ with $1 \leq j \leq k-1$. For any state $x \in \mathbb{Z}_{+}^{n}$, we have

$$
\begin{aligned}
& (1+\lambda) \Delta Q_{j}(x) \leq-\lambda d \frac{Q_{j}(x)}{n}\left(1-\frac{2}{\sqrt{\lambda d}}-\frac{d P_{k-1}(x)}{n}\right)+\frac{Q_{j+1}(x)}{n}, \\
& (1+\lambda) \Delta Q_{j}(x) \geq-\lambda d \frac{Q_{j}(x)}{n}\left(1+\frac{2}{\sqrt{\lambda d}}\right)+\frac{Q_{j+1}(x)}{n} .
\end{aligned}
$$

Proof We begin by calculating

$$
\begin{aligned}
(1+\lambda) \Delta Q_{j}(x)= & \sum_{i=1}^{j} \gamma_{j, i}\left(-\lambda u_{i-1}(x)^{d}+\lambda u_{i}(x)^{d}+u_{i}(x)-u_{i+1}(x)\right) \\
= & \sum_{i=1}^{j} \gamma_{j, i}\left(\lambda\left(1-u_{i-1}(x)^{d}\right)-\lambda\left(1-u_{i}(x)^{d}\right)\right)+\sum_{i=1}^{j} \gamma_{j, i}\left(-\left(1-u_{i}(x)\right)\right. \\
& \left.+\left(1-u_{i+1}(x)\right)\right) .
\end{aligned}
$$

Rearranging now gives

$$
\begin{aligned}
(1+\lambda) \Delta Q_{j}(x)= & \sum_{i=1}^{j}\left(\gamma_{j, i-1}-\gamma_{j, i}\right)\left(1-u_{i}(x)\right) \\
& -\lambda \sum_{i=1}^{j}\left(\gamma_{j, i}-\gamma_{j, i+1}\right)\left(1-u_{i}(x)^{d}\right)+\gamma_{j, j}\left(1-u_{j+1}(x)\right) .
\end{aligned}
$$

Recall that $\gamma_{j, 0}=\gamma_{j, j+1}=0$, and note that $\gamma_{j, 1}>\gamma_{j, 2}>\cdots>\gamma_{j, j}=1$.

As before, we proceed by approximating $1-u_{i}(x)^{d}$ by $d\left(1-u_{i}(x)\right)$, for $i \leq j$. Using first that $1-u_{i}(x)^{d} \leq d\left(1-u_{i}(x)\right)$ for each $i$, we have

$$
\begin{aligned}
(1+\lambda) \Delta Q_{j}(x) \geq & \sum_{i=1}^{j}\left(\gamma_{j, i-1}-\gamma_{j, i}\right)\left(1-u_{i}(x)\right)-\lambda d \sum_{i=1}^{j}\left(\gamma_{j, i}-\gamma_{j, i+1}\right)\left(1-u_{i}(x)\right) \\
& +\left(1-u_{j+1}(x)\right) \\
= & \sum_{i=1}^{j}\left(1-u_{i}(x)\right)\left[\gamma_{j, i-1}+\lambda d \gamma_{j, i+1}-(\lambda d+1) \gamma_{j, i}\right]+\left(1-u_{j+1}(x)\right) \\
= & -\sum_{i=1}^{j}\left(1-u_{i}(x)\right) \gamma_{j, i}\left[\lambda d+1-2 \sqrt{\lambda d} \cos \left(\frac{\pi}{j+1}\right)\right]+\left(1-u_{j+1}(x)\right)
\end{aligned}
$$




$$
\begin{aligned}
& =-\left[\lambda d+1-2 \sqrt{\lambda d} \cos \left(\frac{\pi}{j+1}\right)\right] \frac{Q_{j}(x)}{n}+\left(1-u_{j+1}(x)\right) \\
& \geq-\lambda d \frac{Q_{j}(x)}{n}+\frac{Q_{j+1}(x)}{n}-2 \sqrt{\lambda d} \frac{Q_{j}(x)}{n},
\end{aligned}
$$

as claimed. In the last line above, we used (5.4), as well as the inequality $2 \sqrt{\lambda d} \cos (\pi /(j+$ 1)) $\geq \sqrt{2 \lambda d} \geq 1$, valid since $\lambda d \geq 16$.

For the upper bound, we use the facts that $1-u_{j+1}(x) \leq \frac{Q_{j+1}(x)}{n}$ and $1-u_{i}(x)^{d} \geq$ $d\left(1-u_{i}(x)\right)-\left(\begin{array}{c}d \\ 2\end{array}\right)\left(1-u_{i}(x)\right)^{2}$, to obtain

$$
\begin{aligned}
(1+\lambda) \Delta Q_{j}(x) \leq & -\left[\lambda d+1-2 \sqrt{\lambda d} \cos \left(\frac{\pi}{j+1}\right)\right] \frac{Q_{j}(x)}{n}+\left(1-u_{j+1}(x)\right) \\
& +\lambda\left(\begin{array}{l}
d \\
2
\end{array}\right) \sum_{i=1}^{j}\left(\gamma_{j, i}-\gamma_{j, i+1}\right)\left(1-u_{i}(x)\right)^{2} \\
\leq & -\lambda d \frac{Q_{j}(x)}{n}\left(1-\frac{2}{\sqrt{\lambda d}}\right)+\frac{Q_{j+1}(x)}{n} \\
& +\frac{P_{k-1}(x)}{n} \lambda\left(\begin{array}{l}
d \\
2
\end{array}\right) \sum_{i=1}^{j} \gamma_{j, i}\left(1-u_{i}(x)\right) .
\end{aligned}
$$

This is the result we require, since $\sum_{i=1}^{j} \gamma_{j, i}\left(1-u_{i}(x)\right)=Q_{j}(x) / n$.

We have a similar result for the function $P_{k-1}$. For this function, we need only a fairly crude upper bound on the drift, and we omit the simple proof.

Lemma 5.3 For any state $x \in \mathbb{Z}_{+}^{n}$, we have

$$
(1+\lambda) \Delta P_{k-1}(x) \leq-\frac{\lambda d P_{k-1}(x)}{(k-1) n}+\frac{Q_{k}(x)}{n} .
$$

\section{Hitting Times and Exit Times}

At this point, we begin the proof of Theorem 1.1. Accordingly, from now on we fix values of $\alpha, \beta \in(0,1)$, and a natural number $k$, satisfying $(k-1) \beta<\alpha<k \beta$ and $2 \alpha<1+(k-1) \beta$. Throughout the proof, we consider the $(n, d, \lambda)$-supermarket model with $\lambda=1-n^{-\alpha}$ and $d=n^{\beta}$. (As mentioned in the Introduction, our proofs go through essentially unchanged if we assume only that $1-\lambda=n^{-\alpha+\delta_{1}(n)}$ and $d=n^{\beta+\delta_{2}(n)}$, where $\delta_{1}(n)$ and $\delta_{2}(n)$ tend to zero as $n \rightarrow \infty$, where we replace the expression $n^{-\alpha+(j-1) \beta}$ in the definition of $\mathcal{N}^{\varepsilon}(n, \alpha, \beta)$ below by $(1-\lambda) d^{j-1}$.)

We shall actually prove a result stronger than Theorem 1.1, in that we replace the "tolerance" $1 / \log n$ in that result by a general function $\varepsilon=\varepsilon(n)$. We assume that $\varepsilon(n) \leq 1 / 100$, with $1 / \varepsilon(n)=o\left(n^{\delta}\right)$ for every $\delta>0$, though in fact the proof goes through even if $\varepsilon(n)$ tends to zero as $n^{-\delta}$ for $\delta$ sufficiently small (in terms of $\alpha$ and $\beta$ ).

Accordingly, given $\alpha, \beta \in(0,1)$, and $\varepsilon=\varepsilon(n)$ as above, set $k=\lceil\alpha / \beta\rceil$ as usual, and let $\mathcal{N}^{\varepsilon}(n, \alpha, \beta)$ be the set of queue-lengths vectors $x$ such that $u_{k+1}(x)=0$ and, for $1 \leq j \leq k$,

$$
(1-6 \varepsilon) n^{-\alpha+(j-1) \beta} \leq 1-u_{j}(x) \leq(1+6 \varepsilon) n^{-\alpha+(j-1) \beta} .
$$


Theorem 6.1 Suppose that $\alpha, \beta \in(0,1]$ are constants with $k-1<\alpha / \beta<k$ for some natural number $k$, and that $2 \alpha<1+\beta(k-1)$. Suppose also that $\lambda=\lambda(n)=1-n^{-\alpha}$ and $d=d(n)=n^{\beta}$. Let $\varepsilon=\varepsilon(n) \leq 1 / 100$ be any function such that $\varepsilon(n)^{-1}=o\left(n^{\delta}\right)$ for every $\delta>0$. Then, for $n$ sufficiently large, a copy $\left(Y_{t}\right)$ of the $(n, d, \lambda)$-supermarket process in equilibrium satisfies

$$
\mathbb{P}\left(Y_{t} \notin \mathcal{N}^{\varepsilon}(n, \alpha, \beta)\right) \leq e^{-\frac{1}{4} \log ^{2} n} .
$$

Moreover, if $X_{0} \in \mathcal{N}^{\varepsilon / 6}(n, \alpha, \beta)$, then

$$
\mathbb{P}\left(X_{t} \notin \mathcal{N}^{\varepsilon}(n, \alpha, \beta) \text { for some } t \in\left[0, e^{\frac{1}{3} \log ^{2} n}\right]\right) \leq e^{-\frac{1}{4} \log ^{2} n} .
$$

Theorem 1.1 is the case of Theorem 6.1 with $\varepsilon=1 / 6 \log n$.

The assumptions of Theorem 6.1 assure us that functions of $n$ such as $\varepsilon^{-1} n^{-\alpha+(k-1) \beta} \log ^{2} n$ tend to zero, as the dominant term is the strictly negative power of $n$. We shall use such facts freely throughout the proof, and we shall (sometimes tacitly) assume that $n$ is sufficiently large.

We define a sequence of pairs of subsets of $\mathbb{Z}_{+}^{n}$. Each pair consists of a set $\mathcal{S}_{0}$ in which some inequality holds, and a set $\mathcal{S}_{1}$ in which a looser version of the inequality holds: we also demand that $\mathcal{S}_{0}$ and $\mathcal{S}_{1}$ be subsets of the previous set $\mathcal{R}_{1}$ in the sequence. Associated with each pair $\left(\mathcal{S}_{0}, \mathcal{S}_{1}\right)$ in the sequence is a hitting time

$$
T_{\mathcal{S}}=\inf \left\{t \geq T_{\mathcal{R}}: X_{t} \in \mathcal{S}_{0}\right\}
$$

where $\left(\mathcal{R}_{0}, \mathcal{R}_{1}\right)$ is the previous pair in the sequence, and an exit time

$$
T_{\mathcal{S}}^{\dagger}=\inf \left\{t \geq T_{\mathcal{S}}: X_{t} \notin \mathcal{S}_{1}\right\} .
$$

Our aim in each case is to prove that, with high probability, unless the previous exit time $T_{\mathcal{R}}^{\dagger}$ occurs early, $T_{\mathcal{S}}$ is unlikely to be larger than some quantity $m_{\mathcal{S}}$ whose order is polynomial in $n$. To be precise, if we start in a state in $\mathcal{A}_{0}(\ell, b)$, then the sum of all the $m_{\mathcal{S}}$ is of order at most the maximum of $b n^{1+\alpha}$ and $\ell n$, so if $\ell$ and $b$ are bounded by a polynomial in $n$, then so are all the $m_{\mathcal{S}}$.

Throughout the proof, we set

$$
s_{0}=e^{\frac{1}{3} \log ^{2} n} .
$$

We shall also prove that, again with high probability, each exit time $T_{\mathcal{S}}^{\dagger}$ is at least $s_{0}$, which is larger than the sum of all the terms $m_{\mathcal{S}}$. For convenience, we shall not be too precise about our error probabilities, and simply declare them all to be at most $1 / s_{0}=e^{-\frac{1}{3} \log ^{2} n}$, or some small multiple of $1 / s_{0}$. We will thus prove that, with high probability, we enter each of the sets $\mathcal{S}_{0}$ in turn, while remaining inside all the earlier sets $\mathcal{S}_{1}$.

We fix, for the moment, a pair of positive real numbers $\ell$ and $b$ with $\ell \geq b \geq k$. We set

$$
q(\ell, b)=(22 k+72 b) \varepsilon^{-1} n^{1+\alpha}+8 \ell n,
$$

and we make the (mild) assumption that $\ell \leq e^{\frac{1}{4} \log ^{2} n}$, so that $q(\ell, b) \leq s_{0} / 2$.

The first pair of sets in our sequence will be as defined earlier:

$$
\begin{aligned}
& \mathcal{A}_{0}=\mathcal{A}_{0}(\ell, b)=\left\{x:\|x\|_{\infty} \leq \ell \text { and }\|x\|_{1} \leq b n\right\}, \\
& \mathcal{A}_{1}=\mathcal{A}_{1}(\ell, b)=\left\{x:\|x\|_{\infty} \leq 3 \ell \text { and }\|x\|_{1} \leq 3 b n\right\},
\end{aligned}
$$

and we adopt the hypothesis that $X_{0}=x_{0}$ almost surely, where $x_{0}$ is a fixed state in $\mathcal{A}_{0}=$ $\mathcal{A}_{0}(\ell, b)$, so that $T_{\mathcal{A}}:=\min \left\{t \geq 0: X_{t} \in \mathcal{A}_{0}\right\}=0$. 
For $\ell=\ell^{*}=n^{\alpha} \log ^{2} n$ and $b=b^{*}=2 n^{\alpha}$, Lemma 4.4 tells us that indeed the exit time $T_{\mathcal{A}}^{\dagger}=\inf \left\{t>0: X_{t} \notin \mathcal{A}_{1}\right\}$ is unlikely to be less than $s_{0}$. For smaller values of $\ell$ and $b$, we do not know this a priori.

The sets we define are dependent on the chosen values of $n, \alpha, \beta$ and $\varepsilon$, as well as on $\ell$ and $b$. For the most part, we drop reference to this dependence from the notation. When we need to vary $\varepsilon$ while keeping all other parameters fixed, we shall use the notation (e.g.) $\mathcal{B}_{0}^{\varepsilon}$ to emphasise the dependence. We define:

$$
\begin{aligned}
\mathcal{B}_{0} & =\left\{x: Q_{k}(x) \leq(1+\varepsilon) n(1-\lambda)(\lambda d)^{k-1}\right\} \cap \mathcal{A}_{1}, \\
\mathcal{B}_{1} & =\left\{x: Q_{k}(x) \leq(1+2 \varepsilon) n(1-\lambda)(\lambda d)^{k-1}\right\} \cap \mathcal{A}_{1}, \\
\mathcal{C}_{0} & =\left\{x: P_{k-1}(x) \leq 2 k n(1-\lambda)(\lambda d)^{k-2}\right\} \cap \mathcal{B}_{1}, \\
\mathcal{C}_{1} & =\left\{x: P_{k-1}(x) \leq 3 k n(1-\lambda)(\lambda d)^{k-2}\right\} \cap \mathcal{B}_{1}, \\
\mathcal{D}_{0} & =\left\{x: Q_{k-1}(x) \leq(1+4 \varepsilon) n(1-\lambda)(\lambda d)^{k-2}\right\} \cap \mathcal{C}_{1}, \\
\mathcal{D}_{1} & =\left\{x: Q_{k-1}(x) \leq(1+5 \varepsilon) n(1-\lambda)(\lambda d)^{k-2}\right\} \cap \mathcal{C}_{1}, \\
\mathcal{E}_{0} & =\left\{x: u_{k+1}(x) \leq \varepsilon(1-\lambda) \text { and } Q_{k}(x) \geq(1-3 \varepsilon) n(1-\lambda)(\lambda d)^{k-1}\right\} \cap \mathcal{D}_{1}, \\
\mathcal{E}_{1} & =\left\{x: u_{k+1}(x) \leq \varepsilon(1-\lambda) \text { and } Q_{k}(x) \geq(1-4 \varepsilon) n(1-\lambda)(\lambda d)^{k-1}\right\} \cap \mathcal{D}_{1} .
\end{aligned}
$$

Next we have a sequence of pairs of sets, indexed by $j=k-1, \ldots, 1$ :

$$
\begin{aligned}
\mathcal{G}_{0}^{j} & =\left\{x:\left[1-\left(4+\frac{k-j-1 / 2}{k}\right) \varepsilon\right] n(1-\lambda)(\lambda d)^{j-1} \leq Q_{j}(x)\right. \\
& \left.\leq\left[1+\left(4+\frac{k-j-1 / 2}{k}\right) \varepsilon\right] n(1-\lambda)(\lambda d)^{j-1}\right\} \cap \mathcal{G}_{1}^{j+1}, \\
\mathcal{G}_{1}^{j} & =\left\{x:\left[1-\left(4+\frac{k-j}{k}\right) \varepsilon\right] n(1-\lambda)(\lambda d)^{j-1} \leq Q_{j}(x)\right. \\
& \left.\leq\left[1+\left(4+\frac{k-j}{k}\right) \varepsilon\right] n(1-\lambda)(\lambda d)^{j-1}\right\} \cap \mathcal{G}_{1}^{j+1} .
\end{aligned}
$$

where we declare $\mathcal{G}_{1}^{k}$ to be equal to $\mathcal{E}_{1}$. Finally, departing slightly from our pattern, we define

$$
\mathcal{H}=\mathcal{H}_{0}=\mathcal{H}_{1}=\left\{x: u_{k+1}(x)=0\right\} \cap \mathcal{G}_{1}^{1} .
$$

In the special case $k=1$, only the pairs $\left(\mathcal{B}_{0}, \mathcal{B}_{1}\right),\left(\mathcal{E}_{0}, \mathcal{E}_{1}\right)$ and $\mathcal{H}$ are defined.

The hitting times and exit times are all defined in accordance with the pattern given. For instance $T_{\mathcal{B}}=\inf \left\{t: X_{t} \in \mathcal{B}_{0}\right\}, T_{\mathcal{B}}^{\dagger}=\inf \left\{t>T_{\mathcal{B}}: X_{t} \notin \mathcal{B}_{1}\right\}$, and $T_{\mathcal{C}}=\inf \left\{t \geq T_{\mathcal{B}}: X_{t} \in\right.$ $\mathcal{C}_{0}$. We also set $T_{\mathcal{G}^{k}}=T_{\mathcal{E}}$ and $T_{\mathcal{G}^{k}}^{\dagger}=T_{\mathcal{E}}^{\dagger}$, in accordance with the notion that the set pair $\left(\mathcal{G}_{0}^{k-1}, \mathcal{G}_{1}^{k-1}\right)$ follows $\left(\mathcal{E}_{0}, \mathcal{E}_{1}\right)$ in the sequence.

Initially, the sets above all depend on the values of $\ell$ and $b$ defining the initial pair of sets $\left(\mathcal{A}_{0}, \mathcal{A}_{1}\right)$, since all the sets are intersected with $\mathcal{A}_{1}$. However, since states in $\mathcal{H}$ have no queue of length $k+1$ or greater, we have $\mathcal{H} \subseteq \mathcal{A}_{0}(k, k) \subseteq \mathcal{A}_{1}(\ell, b)$ for all $\ell, b \geq k$, and so the set $\mathcal{H}$ does not depend on $\ell$ and $b$, provided these parameters are each at least $k$.

We claim that $\mathcal{H}^{\varepsilon} \subseteq \mathcal{N}^{\varepsilon}=\mathcal{N}^{\varepsilon}(n, \alpha, \beta)$. Indeed, if $x \in \mathcal{H}^{\varepsilon}$, then

$$
x \in \mathcal{B}_{1} \cap \mathcal{D}_{1} \cap \mathcal{E}_{1} \cap \mathcal{G}_{1}^{k-1} \cap \cdots \cap \mathcal{G}_{1}^{1} \cap\left\{x: u_{k+1}(x)=0\right\} .
$$

This implies that indeed $u_{k+1}(x)=0$, and also that all the $Q_{j}(x)$ are within a factor $1 \pm 5 \varepsilon$ of the values $n(1-\lambda)(\lambda d)^{j-1}$. It now follows from (5.4) and (5.5) that, for each $j=1, \ldots, k$,

$$
\left|\frac{Q_{j}(x)}{n}-\left(1-u_{j}(x)\right)\right| \leq 2(1-\lambda)(\lambda d)^{\frac{1}{2}+j-2} \leq \varepsilon(1-\lambda)(\lambda d)^{j-1},
$$


and so $1-u_{j}(x)$ is within a factor $1 \pm \frac{11}{2} \varepsilon$ of $n(1-\lambda)(\lambda d)^{j-1}$, so that indeed $x \in \mathcal{N}^{\varepsilon}$.

We now state a sequence of lemmas. Throughout, we assume that $X_{0}=x_{0}$ a.s., where $x_{0}$ is an arbitrary state in $\mathcal{A}_{0}=\mathcal{A}_{0}(\ell, b)$.

Lemma 6.2 Let $m_{\mathcal{B}}=8 k \varepsilon^{-1} n(1-\lambda)^{-1}$.

(1) $\mathbb{P}\left(T_{\mathcal{B}} \wedge T_{\mathcal{A}}^{\dagger} \geq m_{\mathcal{B}}\right) \leq 1 / s_{0}$.

(2) $\mathbb{P}\left(T_{\mathcal{B}}^{\dagger} \leq s_{0}<T_{\mathcal{A}}^{\dagger}\right) \leq 1 / s_{0}$.

Lemma 6.3 For $k \geq 2$, let $m_{\mathcal{C}}=8 k n(1-\lambda)^{-1}(\lambda d)^{1-k}$.

(1) $\mathbb{P}\left(T_{\mathcal{C}} \wedge T_{\mathcal{B}}^{\dagger} \geq T_{\mathcal{B}}+m_{\mathcal{C}}\right) \leq 1 / s_{0}$.

(2) $\mathbb{P}\left(T_{\mathcal{C}}^{\dagger} \leq s_{0}<T_{\mathcal{B}}^{\dagger}\right) \leq 1 / s_{0}$.

Lemma 6.4 For $k \geq 2$, let $m_{\mathcal{D}}=8 \varepsilon^{-1} n(1-\lambda)^{-1}(\lambda d)^{-k / 2}$.

(1) $\mathbb{P}\left(T_{\mathcal{D}} \wedge T_{\mathcal{C}}^{\dagger} \geq T_{\mathcal{C}}+m_{\mathcal{D}}\right) \leq 1 / s_{0}$.

(2) $\mathbb{P}\left(T_{\mathcal{D}}^{\dagger} \leq s_{0}<T_{\mathcal{C}}^{\dagger}\right) \leq 1 / s_{0}$.

Lemma 6.5 Let $m_{\mathcal{E}}=m_{\mathcal{E}}(b)=(13 k+72 b) \varepsilon^{-1} n(1-\lambda)^{-1}$.

(1) $\mathbb{P}\left(T_{\mathcal{E}} \wedge T_{\mathcal{D}}^{\dagger} \geq T_{\mathcal{D}}+m_{\mathcal{E}}\right) \leq 1 / s_{0}$.

(2) $\mathbb{P}\left(T_{\mathcal{E}}^{\dagger} \leq s_{0}<T_{\mathcal{D}}^{\dagger}\right) \leq 1 / s_{0}$.

Lemma 6.6 For $k \geq 2$, let $_{\mathcal{G}}=32 k \varepsilon^{-1} n(1-\lambda)^{-1}(\lambda d)^{-1}$. For $j=k-1, \ldots, 1$, we have:

(1) For $j=k-1, \ldots, 1, \mathbb{P}\left(T_{\mathcal{G}^{j}} \wedge T_{\mathcal{G}^{j+1}}^{\dagger} \geq T_{\mathcal{G}^{j+1}}+m_{\mathcal{G}}\right) \leq 1 / s_{0}$;

(2) For $j=k-1, \ldots, 1, \mathbb{P}\left(T_{\mathcal{G}^{j}}^{\dagger} \leq s_{0}<T_{\mathcal{G}^{j+1}}^{\dagger}\right) \leq 1 / s_{0}$.

Lemma 6.7 Let $m_{\mathcal{H}}=m_{\mathcal{H}}(\ell)=n\left(8 \ell+32 \log ^{2} n\right)$.

(1) $\mathbb{P}\left(T_{\mathcal{H}} \wedge T_{\mathcal{G}^{1}}^{\dagger} \geq T_{\mathcal{G}^{1}}+m_{\mathcal{H}}\right) \leq 1 / s_{0}$.

(2) $\mathbb{P}\left(T_{\mathcal{H}}^{\dagger} \leq s_{0}<T_{\mathcal{G}^{1}}^{\dagger}\right) \leq 1 / s_{0}$.

We shall postpone the proofs of these lemmas to later sections. For the remainder of this section, we show how the lemmas imply Theorem 6.1. To start with, combining the lemmas gives the following result.

Proposition 6.8 For any $x_{0} \in \mathcal{A}_{0}=\mathcal{A}_{0}(\ell, b)$, and a copy $\left(X_{t}\right)$ of the process with $X_{0}=x_{0}$ a.s., we have

$$
\mathbb{P}\left(X_{t} \in \mathcal{H} \text { for all } t \in\left[q(\ell, b), s_{0}\right]\right) \geq 1-\frac{2 k+8}{s_{0}}-\mathbb{P}\left(T_{\mathcal{A}}^{\dagger} \leq s_{0}\right) .
$$

Proof The idea is that, with high probability, either the chain $\left(X_{t}\right)$ exits $\mathcal{A}_{1}(\ell, b)$ before time $s_{0}$, or the chain enters each of the sets $\mathcal{B}_{0}, \ldots, \mathcal{H}_{0}$ in turn, within time $q(\ell, b)$, and does not exit any of the sets $\mathcal{A}_{1}, \ldots, \mathcal{H}_{1}$ before time $s_{0}$, which is what we need.

We assume that $k \geq 2$ : if $k=1$, the proof is very similar and shorter. Consider the following list of events concerning the various stopping times we have defined:

$$
\begin{aligned}
& E_{1}=\left\{T_{\mathcal{A}}^{\dagger}>s_{0}\right\}, \quad E_{2}=\left\{T_{\mathcal{B}} \leq m_{\mathcal{B}}\right\}, \quad E_{3}=\left\{T_{\mathcal{B}}^{\dagger}>s_{0}\right\}, \\
& E_{4}=\left\{T_{\mathcal{C}} \leq m_{\mathcal{B}}+m_{\mathcal{C}}\right\}, \quad E_{5}=\left\{T_{\mathcal{C}}^{\dagger}>s_{0}\right\}, \quad E_{6}=\left\{T_{\mathcal{D}} \leq m_{\mathcal{B}}+m_{\mathcal{C}}+m_{\mathcal{D}}\right\}, \\
& E_{7}=\left\{T_{\mathcal{D}}^{\dagger}>s_{0}\right\}, \quad E_{8}=\left\{T_{\mathcal{E}} \leq m_{\mathcal{B}}+\cdots+m_{\mathcal{E}}\right\}, \quad E_{9}=\left\{T_{\mathcal{E}}^{\dagger}>s_{0}\right\},
\end{aligned}
$$




$$
\begin{aligned}
E_{10} & =\left\{T_{\mathcal{G}^{k-1}} \leq m_{\mathcal{B}}+\cdots+m_{\mathcal{E}}+m_{\mathcal{G}}\right\}, \quad E_{11}=\left\{T_{\mathcal{G}^{k-1}}^{\dagger}>s_{0}\right\}, \quad \ldots, \\
E_{2 k+6} & =\left\{T_{\mathcal{G}^{1}} \leq m_{\mathcal{B}}+\cdots+(k-1) m_{\mathcal{G}}\right\}, \quad E_{2 k+7}=\left\{T_{\mathcal{G}^{1}}^{\dagger}>s_{0}\right\}, \\
E_{2 k+8} & =\left\{T_{\mathcal{H}} \leq m_{\mathcal{B}}+\cdots+(k-1) m_{\mathcal{G}}+m_{\mathcal{H}}\right\}, \quad E_{2 k+9}=\left\{T_{\mathcal{H}}^{\dagger}>s_{0}\right\} .
\end{aligned}
$$

If $E_{2 k+8}$ holds, then

$$
\begin{aligned}
T_{\mathcal{H}} \leq & m_{\mathcal{B}}+m_{\mathcal{C}}+m_{\mathcal{D}}+m_{\mathcal{E}}+(k-1) m_{\mathcal{G}}+m_{\mathcal{H}} \\
= & 8 k \varepsilon^{-1} n(1-\lambda)^{-1}+8 k n(1-\lambda)^{-1}(\lambda d)^{1-k} \\
& +8 \varepsilon^{-1} n(1-\lambda)^{-1}(\lambda d)^{-k / 2}+(13 k+72 b) \varepsilon^{-1} n(1-\lambda)^{-1} \\
& +32(k-1) k \varepsilon^{-1} n(1-\lambda)^{-1}(\lambda d)^{-1}+n\left(8 \ell+32 \log ^{2} n\right) \\
\leq & k \varepsilon^{-1} n(1-\lambda)^{-1}\left(8+\frac{8 \varepsilon}{\lambda d}+\frac{8}{\lambda d}+13+\frac{32(k-1)}{\lambda d}\right. \\
& \left.+32 \varepsilon \log ^{2} n(1-\lambda)\right)+72 b \varepsilon^{-1} n(1-\lambda)^{-1}+8 \ell n \\
\leq & \varepsilon^{-1} n(1-\lambda)^{-1}(22 k+72 b)+8 \ell n \\
= & q(\ell, b),
\end{aligned}
$$

for sufficiently large $n$. Therefore, if $E=\bigcap_{j=1}^{2 k+9} E_{j}$ holds, then in particular $E_{2 k+8}$ and $E_{2 k+9}$ hold, which implies that $X_{t} \in \mathcal{H}$ for $q(\ell, b) \leq t \leq s_{0}$. Thus $E$ is contained in the event $\left\{X_{t} \in \mathcal{H}\right.$ for all $\left.t \in\left[q(\ell, b), s_{0}\right]\right\}$, and it suffices to show that $\mathbb{P}(\bar{E}) \leq \frac{2 k+8}{s_{0}}+\mathbb{P}\left(\overline{E_{1}}\right)$. We write

$$
\mathbb{P}(\bar{E})=\mathbb{P}\left(\overline{E_{1}}\right)+\sum_{j=2}^{2 k+9} \mathbb{P}\left(\overline{E_{j}} \cap \bigcap_{i=1}^{j-1} E_{i}\right),
$$

and now we see that it suffices to prove that each of the terms $\mathbb{P}\left(\overline{E_{j}} \cap \bigcap_{i=1}^{j-1} E_{i}\right)$ is at most $1 / s_{0}$.

We show how to derive the first few of these inequalities from Lemmas 6.2-6.7; first we have

$$
\mathbb{P}\left(\overline{E_{2}} \cap E_{1}\right)=\mathbb{P}\left(T_{\mathcal{A}}^{\dagger}>s_{0}, T_{\mathcal{B}}>m_{\mathcal{B}}\right) \leq \mathbb{P}\left(T_{\mathcal{B}} \wedge T_{\mathcal{A}}^{\dagger} \geq m_{\mathcal{B}}\right) \leq 1 / s_{0}
$$

by Lemma 6.2(1). Then we have

$$
\mathbb{P}\left(\overline{E_{3}} \cap E_{1} \cap E_{2}\right) \leq \mathbb{P}\left(\overline{E_{3}} \cap E_{1}\right)=\mathbb{P}\left(T_{\mathcal{B}}^{\dagger} \leq s_{0}<T_{\mathcal{A}}^{\dagger}\right) \leq 1 / s_{0}
$$

by Lemma 6.2(2). Next we have, using the fact that $m_{\mathcal{B}}+m_{\mathcal{C}} \leq s_{0}$,

$$
\begin{aligned}
\mathbb{P}\left(\overline{E_{4}} \cap E_{1} \cap E_{2} \cap E_{3}\right) & \leq \mathbb{P}\left(\overline{E_{4}} \cap E_{2} \cap E_{3}\right) \\
& =\mathbb{P}\left(T_{\mathcal{B}}^{\dagger}>s_{0}, T_{\mathcal{B}} \leq m_{\mathcal{B}}, T_{\mathcal{C}}>m_{\mathcal{B}}+m_{\mathcal{C}}\right) \\
& \leq \mathbb{P}\left(T_{\mathcal{C}} \wedge T_{\mathcal{B}}^{\dagger}>m_{\mathcal{B}}+m_{\mathcal{C}}, T_{\mathcal{B}} \leq m_{\mathcal{B}}\right) \\
& \leq \mathbb{P}\left(T_{\mathcal{C}} \wedge T_{\mathcal{B}}^{\dagger}>T_{\mathcal{B}}+m_{\mathcal{C}}\right) \\
& \leq 1 / s_{0},
\end{aligned}
$$

by Lemma 6.3(1). For $j=5, \ldots, 2 k+9$, the upper bound on $\mathbb{P}\left(\overline{E_{j}} \cap \bigcap_{i=1}^{j-1} E_{i}\right)$ follows either as for $j=3$ or as for $j=4$ : it is important here that $m_{\mathcal{B}}+m_{\mathcal{C}}+m_{\mathcal{D}}+m_{\mathcal{E}}+(k-$ 1) $m_{\mathcal{G}}+m_{\mathcal{H}} \leq q(\ell, b) \leq s_{0}$. 
We now have the following consequence for an equilibrium copy $\left(Y_{t}\right)$ of the $(n, d, \lambda)$ supermarket process.

Corollary 6.9 $\mathbb{P}\left(Y_{t} \in \mathcal{H}\right.$ for all $\left.t \in\left[0, s_{0}\right]\right) \geq 1-(4 k+20) / s_{0} \geq 1-e^{-\frac{1}{4} \log ^{2} n}$, for $n$ sufficiently large.

Proof Recall the definitions of $\ell^{*}$ and $b^{*}$ in Sect. 4. Set also $q^{*}=q\left(\ell^{*}, b^{*}\right)$, and note that $q^{*} \leq s_{0} / 2$, with plenty to spare. From Lemma 4.2, we have that $\mathbb{P}\left(Y_{0} \notin \mathcal{A}_{0}\right) \leq n e^{-\log ^{2} n} \leq$ $e^{-\frac{1}{3} \log ^{2} n}=1 / s_{0}$, since $n \geq 5$. Also, from Lemma 4.4, for a copy $\left(X_{t}^{x}\right)$ of the process starting in a state $x \in \mathcal{A}_{0}$, we have that $\mathbb{P}\left(T_{\mathcal{A}}^{\dagger}<s_{0}\right) \leq 1 / s_{0}$. We now have

$$
\begin{aligned}
\mathbb{P}\left(Y_{t} \notin \mathcal{H} \text { for some } t \in\left[0, s_{0} / 2\right]\right)= & \mathbb{P}\left(Y_{t} \notin \mathcal{H} \text { for some } t \in\left[q^{*}, q^{*}+s_{0} / 2\right]\right) \\
\leq & \mathbb{P}\left(Y_{t} \notin \mathcal{H} \text { for some } t \in\left[q^{*}, q^{*}+s_{0} / 2\right] \mid Y_{0} \in \mathcal{A}_{0}\right) \\
& +\mathbb{P}\left(Y_{0} \notin \mathcal{A}_{0}\right) \\
\leq & \mathbb{P}\left(Y_{t} \notin \mathcal{H} \text { for some } t \in\left[q^{*}, s_{0}\right] \mid Y_{0} \in \mathcal{A}_{0}\right) \\
& +\mathbb{P}\left(Y_{0} \notin \mathcal{A}_{0}\right) \\
\leq & \sup _{x \in \mathcal{A}_{0}^{*}} \mathbb{P}\left(X_{t}^{x} \notin \mathcal{H} \text { for some } t \in\left[q^{*}, s_{0}\right]\right)+\frac{1}{s_{0}} \\
\leq & \frac{2 k+8}{s_{0}}+\frac{1}{s_{0}}+\frac{1}{s_{0}}=\frac{2 k+10}{s_{0}},
\end{aligned}
$$

by Proposition 6.8. Hence $\mathbb{P}\left(Y_{t} \notin \mathcal{H}\right.$ for some $\left.t \in\left[0, s_{0}\right]\right) \leq(4 k+20) / s_{0}$.

The first part of Theorem 6.1 now follows, since we have already noted that $\mathcal{H}^{\varepsilon} \subseteq \mathcal{N}^{\varepsilon}$.

We can also use Corollary 6.9 to prove the following more explicit version of Proposition 6.8 .

Theorem 6.10 Suppose that $\ell$ and $b$ are at least $k$, and that $q(\ell, b) \leq s_{0} / 2$. Let $x_{0}$ be any queue-lengths vector in $\mathcal{A}_{0}(\ell, b)$, and suppose that $X_{0}=x_{0}$ a.s. Then we have, for $n$ sufficiently large,

$$
\mathbb{P}\left(X_{t} \in \mathcal{H} \text { for all } t \in\left[q(\ell, b), s_{0}\right]\right) \geq 1-\frac{6 k+28}{s_{0}} \geq 1-e^{-\frac{1}{4} \log ^{2} n} .
$$

Proof We apply, successively, Proposition 6.8, Lemma 4.3 and Corollary 6.9 to obtain that

$$
\begin{aligned}
\mathbb{P}\left(X_{t} \in \mathcal{H} \text { for all } t \in\left[q(\ell, b), s_{0}\right]\right) & \geq 1-\frac{2 k+8}{s_{0}}-\mathbb{P}\left(T_{\mathcal{A}}^{\dagger} \leq s_{0}\right) \\
& =1-\frac{2 k+8}{s_{0}}-\mathbb{P}\left(\exists t \in\left[0, s_{0}\right], X_{t} \notin \mathcal{A}_{1}(\ell, b)\right) \\
& \geq 1-\frac{2 k+8}{s_{0}}-\mathbb{P}\left(\exists t \in\left[0, s_{0}\right], Y_{t} \notin \mathcal{A}_{0}(\ell, b)\right) \\
& \geq 1-\frac{2 k+8}{s_{0}}-\mathbb{P}\left(\exists t \in\left[0, s_{0}\right], Y_{t} \notin \mathcal{H}\right) \\
& \geq 1-\frac{2 k+8}{s_{0}}-\frac{4 k+20}{s_{0}},
\end{aligned}
$$

as required. 
To see the final assertion of Theorem 6.1, suppose that $X_{0}=x_{0}$ a.s., where $x_{0}$ is in the set

$$
\mathcal{I}=\mathcal{A}_{0} \cap \mathcal{B}_{0} \cap \mathcal{C}_{0} \cap \mathcal{D}_{0} \cap \mathcal{E}_{0} \cap \bigcap_{j=1}^{k-1} \mathcal{G}_{0}^{j} \cap \mathcal{H}_{0} .
$$

Then all the hitting times $T_{\mathcal{B}}, T_{\mathcal{C}}, T_{\mathcal{D}}, T_{\mathcal{E}}, T_{\mathcal{G}}^{j}$ and $T_{\mathcal{H}}$ are equal to 0. In the notation of the proof of Proposition 6.8, this implies that the events $E_{j}$ for $j$ even occur with probability 1. Also, by Lemma $4.4, \mathbb{P}\left(\overline{E_{1}}\right) \leq 1 / s_{0}$. So following the proof of Proposition 6.8 yields that, for $X_{0}=x_{0} \in \mathcal{I}$,

$$
\mathbb{P}\left(X_{t} \in \mathcal{H} \text { for all } t \in\left[0, s_{0}\right]\right) \geq 1-(k+5) / s_{0} \geq 1-e^{-\frac{1}{4} \log ^{2} n} .
$$

It can easily be seen that $\mathcal{N}^{\varepsilon / 6} \subseteq \mathcal{I}^{\varepsilon}$, and hence this result completes the proof of Theorem 6.1.

\section{Proofs of Lemmas 6.2, 6.3 and 6.4}

In this section, we prove the first three of the sequence of lemmas stated in the previous section, and also derive tighter inequalities on the drifts of the functions $Q_{j}(x)$ for $x \in \mathcal{D}_{1}$. The proofs of the three lemmas are all straightforward applications of Lemma 3.3, and all similar to one another.

\section{Proof of Lemma 6.2}

Proof We apply Lemma 3.3. We set $\left(\varphi_{t}\right)=\left(\mathcal{F}_{t}\right)$, the natural filtration of the process, and also: $F=Q_{k}, \mathcal{S}=\mathcal{A}_{1}, p=1$,

$$
h=(1+\varepsilon)(1-\lambda) n(\lambda d)^{k-1}, \quad \rho=\varepsilon(1-\lambda) n(\lambda d)^{k-1},
$$

$m=m_{\mathcal{B}}=8 k \varepsilon^{-1} n(1-\lambda)^{-1}, s=s_{0}=e^{\frac{1}{3} \log ^{2} n}$ and $T^{*}=0$. It is clear that $\rho \geq 2$ and that $Q_{k}(x) \leq c:=k n$ for any $x \in \mathbb{Z}_{+}^{n}$. We note also that $Q_{k}$ takes jumps of size at most 1 .

Suppose now that $Q_{k}(x) \geq h$. Then

$$
\exp \left(-\frac{d Q_{k}\left(X_{t}\right)}{k n}\right) \leq \exp \left(-\frac{(1-\lambda)(\lambda d)^{k}}{k}\right) \leq \frac{\varepsilon(1-\lambda)}{4} .
$$

The final inequality above is true comfortably, as $(1-\lambda) d^{k}=n^{-\alpha+k \beta}=n^{\delta}$ for some $\delta>0$.

Hence, by Lemma 5.1, for $x$ with $Q_{k}(x) \geq h$, we have

$$
\begin{aligned}
(1+\lambda) \Delta Q_{k}(x) \leq & \beta_{k}\left((1-\lambda)-u_{k+1}(x)+\lambda \exp \left(-d Q_{k}(x) / k n\right)\right) \\
& -\frac{1}{(\lambda d)^{k-1}} \frac{Q_{k}(x)}{n}\left(1-\frac{2}{\lambda d}\right) \\
\leq & \beta_{k}\left((1-\lambda)+\lambda \frac{\varepsilon(1-\lambda)}{4}\right)-(1+\varepsilon)(1-\lambda)(1-\varepsilon / 5) \\
\leq & (1-\lambda)\left[1+\frac{\varepsilon}{4}-(1+3 \varepsilon / 4)\right]=-(1-\lambda) \frac{\varepsilon}{2} .
\end{aligned}
$$

So $\Delta Q_{k}(x) \leq-(1-\lambda) \varepsilon / 4:=-v$. Note that $m_{\mathcal{B}} v=2 c$.

We have now verified that the conditions of Lemma 3.3 are satisfied, for the given values of the parameters. As in the lemma, we have $T_{0}=T_{\mathcal{A}}^{\dagger}, T_{1}=\inf \left\{t: Q_{k}\left(X_{t}\right) \leq h\right\}$ and $T_{2}=\inf \left\{t>T_{1}: Q_{k}\left(X_{t}\right) \geq h+\rho\right\}$. 
It need not be the case that $T_{1}=T_{\mathcal{B}}$, since $X_{T_{1}}$ need not be in $\mathcal{A}_{1}$. However, we do have $T_{1} \wedge T_{\mathcal{A}}^{\dagger}=T_{\mathcal{B}} \wedge T_{\mathcal{A}}^{\dagger}$ and thus

$$
\begin{aligned}
\mathbb{P}\left(T_{\mathcal{B}} \wedge T_{\mathcal{A}}^{\dagger}>m_{\mathcal{B}}\right) & =\mathbb{P}\left(T_{1} \wedge T_{\mathcal{A}}^{\dagger}>m_{\mathcal{B}}\right) \\
& \leq \exp \left(-v^{2} m_{\mathcal{B}} / 8\right) \\
& =\exp (-\varepsilon k n(1-\lambda) / 16) \leq 1 / s_{0}
\end{aligned}
$$

Also the events $T_{2} \leq s_{0}<T_{\mathcal{A}}^{\dagger}$ and $T_{\mathcal{B}}^{\dagger} \leq s_{0}<T_{\mathcal{A}}^{\dagger}$ coincide, so we have

$$
\begin{aligned}
\mathbb{P}\left(T_{\mathcal{B}}^{\dagger} \leq s_{0}<T_{\mathcal{A}}^{\dagger}\right) & \leq \mathbb{P}\left(T_{2} \leq s_{0}<T_{\mathcal{A}}^{\dagger}\right) \\
& \leq \frac{100 s}{v^{2}} \exp (-\rho v / 8) \\
& =\frac{100 s_{0}}{v^{2}} \exp \left(-\varepsilon^{2}(1-\lambda)^{2} n(\lambda d)^{k-1} / 32\right) \\
& =\frac{100 s_{0}}{v^{2}} \exp \left(-\varepsilon^{2} \lambda^{k-1} n^{1-2 \alpha+(k-1) \beta} / 32\right) \\
& \leq 1 / s_{0},
\end{aligned}
$$

as required. Here we used that $1-2 \alpha+(k-1) \beta>0$.

\section{Proof of Lemma 6.3}

Proof Again we apply Lemma 3.3 to the Markov process $\left(X_{t}\right)$ with its natural filtration. Set $F=P_{k-1}, \mathcal{S}=\mathcal{B}_{1}, p=1$,

$$
h=2 k n(1-\lambda)(\lambda d)^{k-2}, \rho=k n(1-\lambda)(\lambda d)^{k-2},
$$

$m=m_{\mathcal{C}}=8 k n(1-\lambda)^{-1}(\lambda d)^{1-k}$, and $s=s_{0}$. Set $T^{*}=T_{\mathcal{B}}$. It is again clear that $\rho \geq 2$, that $P_{k-1}$ takes jumps of size at most 1 , and that $P_{k-1}(x) \leq c:=k n$ for all $x \in \mathbb{Z}_{+}^{n}$. Here $T_{0}=T_{\mathcal{B}}^{\dagger}, T_{1}=\inf \left\{t \geq T_{\mathcal{B}}: P_{k-1}\left(X_{t}\right) \leq h\right\}$, and $T_{2}=\inf \left\{t>T_{1}: P_{k-1}\left(X_{t}\right) \geq h+\rho\right\}$.

For $x \in \mathcal{B}_{1}$ with $P_{k-1}(x) \geq h$, we have $Q_{k}(x) \leq(1+2 \varepsilon) n(1-\lambda)(\lambda d)^{k-1}$ and so, by Lemma 5.3,

$$
\begin{aligned}
(1+\lambda) \Delta P_{k-1}(x) & \leq-\frac{\lambda d P_{k-1}(x)}{(k-1) n}+\frac{Q_{k}(x)}{n} \\
& \leq-2 \lambda d(1-\lambda)(\lambda d)^{k-2}+(1+2 \varepsilon)(1-\lambda)(\lambda d)^{k-1} \\
& \leq-\frac{1}{2}(1-\lambda)(\lambda d)^{k-1}
\end{aligned}
$$

We conclude that, for $\operatorname{such} x, \Delta P_{k-1}(x) \leq-\frac{1}{4}(1-\lambda)(\lambda d)^{k-1}:=-v$. Note that $m_{\mathcal{C}} v=2 c$.

As in the previous lemma, it need not be the case that $T_{1}=T_{\mathcal{C}}$, since $X_{T_{1}}$ need not be in $\mathcal{B}_{1}$, so we may have $T_{\mathcal{C}}>T_{1}$. However, we do have $T_{1} \wedge T_{\mathcal{B}}^{\dagger}=T_{\mathcal{C}} \wedge T_{\mathcal{B}}^{\dagger}$. From Lemma 3.3, we obtain that

$$
\begin{aligned}
\mathbb{P}\left(T_{\mathcal{C}} \wedge T_{\mathcal{B}}^{\dagger}>T_{\mathcal{B}}+m_{\mathcal{C}}\right) & =\mathbb{P}\left(T_{1} \wedge T_{0}>T_{\mathcal{B}}+m_{\mathcal{C}}\right) \\
& \leq \exp \left(-v^{2} m_{\mathcal{C}} / 8\right) \\
& =\exp \left(-k n(1-\lambda)(\lambda d)^{k-1} / 16\right) \leq 1 / s_{0} .
\end{aligned}
$$

Similarly, the events $T_{2} \leq s_{0}<T_{\mathcal{B}}^{\dagger}$ and $T_{\mathcal{C}}^{\dagger} \leq s_{0}<T_{\mathcal{B}}^{\dagger}$ coincide, and so, for $k \geq 2$, 


$$
\begin{aligned}
\mathbb{P}\left(T_{\mathcal{C}}^{\dagger} \leq s_{0}<T_{\mathcal{B}}^{\dagger}\right) & =\mathbb{P}\left(T_{2} \leq s_{0}<T_{0}\right) \\
& \leq \frac{100 s_{0}}{v^{2}} \exp (-\rho v / 8) \\
& =\frac{100 s_{0}}{v^{2}} \exp \left(-k n(1-\lambda)^{2}(\lambda d)^{2 k-3} / 32\right) \\
& \leq \frac{100 s_{0}}{v^{2}} \exp \left(-k \lambda^{2 k-3} n^{1-2 \alpha+(k-1) \beta+(k-2) \beta} / 32\right) \\
& \leq 1 / s_{0},
\end{aligned}
$$

as required.

\section{Sketch of Proof of Lemma 6.4}

Proof The basic plan for this proof is the same as for the previous two lemmas, but here we have to take account of the fact that $Q_{k-1}$ can take jumps of size up to $(\lambda d)^{(k-2) / 2}$, and accordingly we apply Lemma 3.3 to the "scaled" function $F(x)=Q_{k-1}^{\prime}(x)=$ $Q_{k-1}(x) /(\lambda d)^{(k-2) / 2}$.

Apart from this, the proof is identical in structure to that of Lemma 6.3, and we give only the key calculation. For $x \in \mathcal{C}_{1}$ with $Q_{k-1}^{\prime}(x) \geq h=(1+4 \varepsilon) n(1-\lambda)(\lambda d)^{(k-2) / 2}$, we have $Q_{k}(x) \leq(1+2 \varepsilon) n(1-\lambda)(\lambda d)^{k-1}, P_{k-1}(x) \leq 3 k n(1-\lambda)(\lambda d)^{k-2}$ and $Q_{k-1}(x) \geq$ $(1+4 \varepsilon) n(1-\lambda)(\lambda d)^{k-2}$. Thus, by Lemma 5.2 with $j=k-1$, we have

$$
\begin{aligned}
(1+\lambda) \Delta Q_{k-1}(x) \leq & -\lambda d \frac{Q_{k-1}(x)}{n}\left(1-\frac{2}{\sqrt{\lambda d}}-\frac{d P_{k-1}(x)}{n}\right)+\frac{Q_{k}(x)}{n}, \\
\leq & -\lambda d(1+4 \varepsilon)(1-\lambda)(\lambda d)^{k-2}\left(1-\frac{2}{\sqrt{\lambda d}}-3 k d(1-\lambda)(\lambda d)^{k-2}\right) \\
& +(1+2 \varepsilon)(1-\lambda)(\lambda d)^{k-1} \\
\leq & -\varepsilon(1-\lambda)(\lambda d)^{k-1} .
\end{aligned}
$$

Thus, for such $x$, the drift in the scaled chain satisfies $\Delta Q_{k-1}^{\prime}(x) \leq-\frac{1}{2} \varepsilon(1-\lambda)(\lambda d)^{k / 2}:=$ $-v$. Now $Q_{k-1}^{\prime}(x) \leq c:=2 n$ for all $x$ by (5.3), and $m_{\mathcal{D}} v=2 c$.

It is now straightforward to derive the result.

A queue-lengths vector $x \in \mathcal{D}_{1}$ satisfies the three inequalities:

$$
\begin{aligned}
Q_{k}(x) & \leq(1+2 \varepsilon) n(1-\lambda)(\lambda d)^{k-1}, \\
P_{k-1}(x) & \leq 3 k n(1-\lambda)(\lambda d)^{k-2}, \\
Q_{k-1}(x) & \leq(1+5 \varepsilon) n(1-\lambda)(\lambda d)^{k-2} ;
\end{aligned}
$$

in fact the second of these is redundant, as $P_{k-1}(x) \leq Q_{k-1}(x) \leq 2 n(1-\lambda)(\lambda d)^{k-2}$ for all $x \in \mathbb{Z}_{+}^{n}$. Substituting these bounds into the bounds of Lemmas 5.1 and 5.2, we obtain the following.

Lemma 7.1 For $x \in \mathcal{D}_{1}$, we have

$$
\begin{aligned}
(1+\lambda) \Delta Q_{k}(x) \leq & \beta_{k}\left(1-\lambda-u_{k+1}(x)\right)-\frac{Q_{k}(x)}{n(\lambda d)^{k-1}} \\
& +\exp \left(-d Q_{k}(x) / k n\right)+\frac{\varepsilon}{6}(1-\lambda), \\
(1+\lambda) \Delta Q_{k}(x) \geq & \beta_{k}\left(1-\lambda-u_{k+1}(x)\right)-\frac{Q_{k}(x)}{n(\lambda d)^{k-1}}-\frac{\varepsilon}{6}(1-\lambda),
\end{aligned}
$$


and, for $1 \leq j \leq k-1$,

$$
\begin{aligned}
& (1+\lambda) \Delta Q_{j}(x) \leq-\lambda d \frac{Q_{j}(x)}{n}\left(1-\frac{\varepsilon}{25 k}\right)+\frac{Q_{j+1}(x)}{n}, \\
& (1+\lambda) \Delta Q_{j}(x) \geq-\lambda d \frac{Q_{j}(x)}{n}\left(1+\frac{\varepsilon}{50 k}\right)+\frac{Q_{j+1}(x)}{n} .
\end{aligned}
$$

\section{Proof of Lemma 6.5}

This section is devoted to the rather more complex proof of Lemma 6.5. First, we prove a statement stronger than part (1) of the lemma. We set

$$
\begin{aligned}
\mathcal{K} & =\left\{x: u_{k+1}(x) \leq \varepsilon(1-\lambda) \text { and } Q_{k}(x) \geq n\left(1-\frac{\varepsilon}{3}\right)(1-\lambda)(\lambda d)^{k-1}\right\} \cap \mathcal{D}_{1} ; \\
W_{\mathcal{K}} & =\inf \left\{t \geq T_{\mathcal{D}}: X_{t} \in \mathcal{K}\right\} .
\end{aligned}
$$

Note that $\mathcal{K} \subseteq \mathcal{E}_{0}$, so to prove Lemma 6.5(1) it suffices to prove that

$$
\mathbb{P}\left(W_{\mathcal{K}} \wedge T_{\mathcal{D}}^{\dagger} \geq T_{\mathcal{D}}+m_{\mathcal{E}}\right) \leq 1 / s_{0} .
$$

We prove this result on the assumption that $T_{\mathcal{D}}=0$ (i.e., that $x_{0} \in \mathcal{A}_{0} \cap \mathcal{B}_{0} \cap \mathcal{C}_{0} \cap \mathcal{D}_{0}$ ). The general case follows immediately by applying the result for $T_{\mathcal{D}}=0$ to the shifted process $\left(X_{t}^{\prime}\right)=\left(X_{T_{\mathcal{D}}+t}\right)$, using the strong Markov property. So our task is to show that $\mathbb{P}\left(W_{\mathcal{K}} \wedge T_{\mathcal{D}}^{\dagger} \geq m_{\mathcal{E}}\right) \leq 1 / s_{0}$, where $W_{\mathcal{K}}=\inf \left\{t \geq 0: X_{t} \in \mathcal{K}\right\}$, whenever $X_{0}=x_{0}$ a.s., for any $x_{0} \in \mathcal{A}_{0} \cap \mathcal{B}_{0} \cap \mathcal{C}_{0} \cap \mathcal{D}_{0}$.

We define the following further sets, hitting times and exit times. We set

$$
\begin{aligned}
\mathcal{L}_{1}^{k+1} & =\mathcal{D}_{1} \backslash \mathcal{K} \\
& =\left\{x: u_{k+1}(x)>\varepsilon(1-\lambda) \text { or } Q_{k}(x)<n\left(1-\frac{\varepsilon}{3}\right)(1-\lambda)(\lambda d)^{k-1}\right\} \cap \mathcal{D}_{1},
\end{aligned}
$$

$W_{\mathcal{L}^{k+1}}=0$ and $W_{\mathcal{L}^{k+1}}^{\dagger}=\inf \left\{t \geq 0: X_{t} \notin \mathcal{L}_{1}^{k+1}\right\}=W_{\mathcal{K}} \wedge T_{\mathcal{D}}^{\dagger}$. Also, for $j=k, \ldots, 1$, let

$$
\begin{aligned}
\mathcal{L}_{0}^{j} & =\left\{x: Q_{j}(x) \leq n(1-\lambda)(\lambda d)^{j-1}\left(1-\frac{\varepsilon}{6}-\frac{j \varepsilon}{6 k}\right)\right\} \cap \mathcal{L}_{1}^{j+1} ; \\
\mathcal{L}_{1}^{j} & =\left\{x: Q_{j}(x) \leq n(1-\lambda)(\lambda d)^{j-1}\left(1-\frac{\varepsilon}{6}-\frac{j \varepsilon}{6 k}+\frac{\varepsilon}{24 k}\right)\right\} \cap \mathcal{L}_{1}^{j+1} ; \\
W_{\mathcal{L}^{j}} & =\inf \left\{t \geq W_{\mathcal{L}^{j+1}}: X_{t} \in \mathcal{L}_{0}^{j}\right\} ; \\
W_{\mathcal{L}^{j}}^{\dagger} & \left.=\inf \left\{t \geq W_{\mathcal{L}^{j}}: X_{t} \notin \mathcal{L}_{1}^{j}\right)\right\} .
\end{aligned}
$$

Our goal is to show that $\mathbb{P}\left(W_{\mathcal{L}^{k+1}}^{\dagger}<m_{\mathcal{E}}\right) \geq 1-1 / s_{0}$. If $x_{0} \in \mathcal{K}$, then $W_{\mathcal{L}^{k+1}}^{\dagger}=0$ and we are done, so we may assume that $x_{0} \notin \mathcal{K}$, and hence that $x_{0} \in \mathcal{L}_{1}^{k+1}$. Thus Lemma 6.5(1) follows from the proposition below.

Proposition 8.1 Let $x_{0}$ be any queue-lengths vector in $\mathcal{L}_{1}^{k+1}$. For a copy $\left(X_{t}\right)$ of the $(n, d, \lambda)$ supermarket process with $X_{0}=x_{0}$ a.s., we have

$$
\mathbb{P}\left(W_{\mathcal{L}^{k+1}}^{\dagger} \geq m_{\mathcal{E}}\right) \leq 1 / s_{0} .
$$

For the proof of Proposition 8.1, we fix a state $x_{0} \in \mathcal{L}_{1}^{k+1}$, and work with a copy $\left(X_{t}\right)$ of the $(n, d, \lambda)$-supermarket process where $X_{0}=x_{0}$ a.s. 
Our general plan for proving Proposition 8.1 is as follows. We suppose that the process $\left(X_{t}\right)$ stays inside $\mathcal{L}_{1}^{k+1}=\mathcal{D}_{1} \backslash \mathcal{K}$ over the interval $\left[0, m_{\mathcal{E}}\right.$ ), with the aim of showing that this event has low probability. Observe that, if $x \in \mathcal{L}_{1}^{k+1} \backslash \mathcal{L}_{0}^{k}$, then $u_{k+1}(x)>\varepsilon(1-\lambda)$ and $Q_{k}(x)>n\left(1-\frac{\varepsilon}{3}\right)(1-\lambda)(\lambda d)^{k-1}$. This "excess" in $u_{k+1}$ would result in a downward drift in $Q_{k}\left(X_{t}\right)$, so if the process does not exit $\mathcal{L}_{1}^{k+1}$ quickly, then it enters $\mathcal{L}_{0}^{k}$ quickly, and stays in $\mathcal{L}_{1}^{k}$ throughout the interval $\left[0, m_{\mathcal{E}}\right)$ : i.e., $W_{\mathcal{L}^{k}}$ is small and $W_{\mathcal{L}^{k}}^{\dagger}$ is large, with high probability. This means that $Q_{k}\left(X_{t}\right)$ maintains a "deficit" compared to $\tilde{Q}_{k}:=n(1-\lambda)(\lambda d)^{k-1}$ until time $m_{\mathcal{E}}$. A deficit in $Q_{k}\left(X_{t}\right)$ would lead to a deficit in each $Q_{j}\left(X_{t}\right)$ in turn, compared to $\tilde{Q}_{j}:=n(1-\lambda)(\lambda d)^{j-1}$, for $j=k-1, k-2, \ldots, 1$ : each $W_{\mathcal{L}^{j}}$ is small, and $W_{\mathcal{L}^{j}}^{\dagger}$ is large, with high probability. Finally, a deficit in $Q_{1}\left(X_{t}\right)$ compared to $\tilde{Q}_{1}=n(1-\lambda)$ is unsustainable, as this would lead to a drift down in the total number of customers over a long enough time interval to empty the entire system of customers. This would entail exiting the set $\mathcal{B}_{1} \supseteq \mathcal{L}_{1}^{k+1}$, a contradiction.

Lemma 8.2 (1) $\mathbb{P}\left(W_{\mathcal{L}^{k}} \wedge W_{\mathcal{L}^{k+1}}^{\dagger} \geq 12 k \varepsilon^{-1} n(1-\lambda)^{-1}\right) \leq 1 / 6 s_{0}$.

(2) $\mathbb{P}\left(W_{\mathcal{L}^{k}}^{\dagger}<m_{\mathcal{E}} \leq W_{\mathcal{L}^{k+1}}^{\dagger}\right) \leq 1 / 12 s_{0}$.

Proof We apply Lemma 3.3 to the process $\left(X_{t}\right)$, with its natural filtration, and the function $F=Q_{k}$. We set $h=\left(1-\frac{\varepsilon}{3}\right) n(1-\lambda)(\lambda d)^{k-1}$ and $\rho=\frac{\varepsilon}{24 k} n(1-\lambda)(\lambda d)^{k-1} \geq 2$. We also set $\mathcal{S}=\mathcal{L}_{1}^{k+1}$ and $T^{*}=0$. We note that $Q_{k}(x) \leq c:=k n$ for every $x$, and we take $m=12 k \varepsilon^{-1} n(1-\lambda)^{-1}$, and $s=m_{\mathcal{E}}-1$. Then $T_{0}=W_{\mathcal{L}^{k+1}}^{\dagger}, T_{1}=\inf \left\{t: Q_{k}\left(X_{t}\right) \leq h\right\}$ and $T_{2}=\inf \left\{t>T_{1}: Q_{k}\left(X_{t}\right) \geq h+\rho\right\}$, as in the lemma.

For $x \in \mathcal{L}_{1}^{k+1}$ with $Q_{k}(x)>h$, we have $u_{k+1}(x)>\varepsilon(1-\lambda)$ and $x \in \mathcal{D}_{1}$. So Lemma 7.1 applies, and we have

$$
\begin{aligned}
(1+\lambda) \Delta Q_{k}(x) & \leq \beta_{k}\left(1-\lambda-u_{k+1}\left(X_{t}\right)\right)-\frac{Q_{k}(x)}{n(\lambda d)^{k-1}}+\exp \left(-d Q_{k}(x) / k n\right)+\frac{\varepsilon}{6}(1-\lambda) \\
& \leq(1-\lambda)(1-\varepsilon)-(1-\lambda)\left(1-\frac{\varepsilon}{3}\right)+\frac{\varepsilon}{6}(1-\lambda)+\frac{\varepsilon}{6}(1-\lambda) \\
& =-\frac{1}{3} \varepsilon(1-\lambda) .
\end{aligned}
$$

So $\Delta Q_{k}(x) \leq-\frac{1}{6} \varepsilon(1-\lambda):=-v$ for such $x$. Note that $m v=2 c$. Hence we may apply Lemma 3.3.

As in earlier lemmas, we have $T_{1} \wedge W_{\mathcal{L}^{k+1}}^{\dagger}=W_{\mathcal{L}^{k}} \wedge W_{\mathcal{L}^{k+1}}^{\dagger}$, so we obtain

$$
\begin{aligned}
\mathbb{P}\left(W_{\mathcal{L}^{k}} \wedge W_{\mathcal{L}^{k+1}}^{\dagger}>m\right) & =\mathbb{P}\left(T_{1} \wedge T_{0}>m\right) \\
& \leq \exp \left(-v^{2} m / 8\right) \\
& =\exp (-\varepsilon k n(1-\lambda) / 24)<1 / 6 s_{0} .
\end{aligned}
$$

Also the events $W_{\mathcal{L}^{k}}^{\dagger}<m_{\mathcal{E}} \leq W_{\mathcal{L}^{k+1}}^{\dagger}$ and $T_{2}<m_{\mathcal{E}} \leq W_{\mathcal{L}^{k+1}}^{\dagger}$ coincide, and the second is equivalent to $T_{2} \leq s<W_{\mathcal{L}^{k+1}}^{\dagger}$ (since $\left.s=m_{\mathcal{E}}-1\right)$. So

$$
\begin{aligned}
\mathbb{P}\left(W_{\mathcal{L}^{k}}^{\dagger}<m_{\mathcal{E}} \leq W_{\mathcal{L}^{k+1}}^{\dagger}\right) & =\mathbb{P}\left(T_{2} \leq s<T_{0}\right) \\
& \leq \frac{100 s}{v^{2}} \exp (-\rho v / 8)
\end{aligned}
$$




$$
\begin{aligned}
& =\frac{100 s}{v^{2}} \exp \left(-\varepsilon^{2} n(1-\lambda)^{2}(\lambda d)^{k-1} / 1152 k\right) \\
& <1 / 12 s_{0}
\end{aligned}
$$

as required.

The next lemma states that, if the process stays in some set $\mathcal{L}_{1}^{j+1}$ for a long time, then it quickly enters the "next" set $\mathcal{L}_{0}^{j}$, and stays in $\mathcal{L}_{1}^{j}$ for a long time.

Lemma 8.3 For each $j=k-1, \ldots, 1$,

(1) $\mathbb{P}\left(W_{\mathcal{L}^{j+1}}^{\dagger} \wedge W_{\mathcal{L}^{j}}>W_{\mathcal{L}^{j+1}}+\varepsilon^{-1} n(1-\lambda)^{-1}\right) \leq 1 / 3 k s_{0}$.

(2) $\mathbb{P}\left(W_{\mathcal{L}^{j}}^{\dagger}<m_{\mathcal{E}} \leq W_{\mathcal{L}^{j+1}}^{\dagger}\right) \leq 1 / 3 k s_{0}$.

Proof (Sketch) This proof is very similar to that of earlier lemmas, and we mention only a few points. As in Lemma 6.4, we apply Lemma 3.3 to the scaled process $Q_{j}^{\prime}(x)=$ $Q_{j}(x) /(\lambda d)^{(j-1) / 2}$. The key step is to show that, for $x \in \mathcal{L}_{1}^{j+1}$ with $Q_{j}^{\prime} \geq h=$ $n(1-\lambda)(\lambda d)^{(j-1) / 2}\left(1-\frac{\varepsilon}{6}-\frac{j \varepsilon}{6 k}\right)$, we have $\Delta Q_{j}^{\prime}(x) \leq-\frac{\varepsilon}{24 k}(1-\lambda)(\lambda d)^{(j+1) / 2}:=-v$. The proof now proceeds as earlier ones.

For part (2) of the lemma, we set $\rho=\frac{\varepsilon}{24 k} n(1-\lambda)(\lambda d)^{(j-1) / 2}$. We make use of the fact that the value of $Q_{j}^{\prime}$ only changes if either (i) the event is an arrival, and some queue of length at most $j-1$ is inspected, or (ii) the event is a departure from some queue of length at most $j$. From any state $x \in \mathcal{L}_{1}^{j}$, the probability of (i) is at most $d\left(1-u_{j}(x)\right) \leq d Q_{j}(x) / n \leq(1-\lambda) d^{j}$, and the probability of (ii) is at most $\left(1-u_{j+1}(x)\right) \leq Q_{j+1}(x) / n \leq(1-\lambda) d^{j}$. Hence we may apply Lemma 3.3(ii), with $p=2(1-\lambda) d^{j}$.

$$
\begin{aligned}
\mathbb{P}\left(W_{\mathcal{L}^{j}}^{\dagger}<m_{\mathcal{E}} \leq W_{\mathcal{L}^{j+1}}^{\dagger}\right) & \leq \frac{100 m_{\mathcal{E}}}{v^{2}} \exp (-\rho v / 8 p) \\
& =\frac{100 m_{\mathcal{E}}}{v^{2}} \exp \left(-\frac{\varepsilon^{2} \lambda^{j}}{4608 k^{2}} n(1-\lambda)\right) \\
& \leq 1 / 3 k s_{0} .
\end{aligned}
$$

We now prove a hitting time lemma for $\left\|X_{t}\right\|_{1}$, the total number of customers in the system at time $t$. Let $W_{\mathcal{M}}=\min \left\{t \geq W_{\mathcal{L}^{1}}:\left\|X_{t}\right\|_{1}=0\right\}$.

\section{Lemma 8.4}

$$
\mathbb{P}\left(W_{\mathcal{L}^{1}}^{\dagger} \wedge W_{\mathcal{M}}>W_{\mathcal{L}^{1}}+72 b \varepsilon^{-1} n(1-\lambda)^{-1}\right) \leq 1 / 12 s_{0} .
$$

Proof We apply Lemma 3.3(i) to the chain $\left(X_{t}\right)$, with the filtration $\left(\mathcal{F}_{t}\right)$, and the function $F(x)=\|x\|_{1}$, which takes jumps of size at most 1 . Since $\mathcal{A}_{1}(\ell, b) \supseteq \mathcal{L}_{1}^{1}$, we have $\left\|X_{0}\right\|_{1} \leq$ $c:=3 b n$. We also set $\mathcal{S}=\mathcal{L}_{1}^{1}, T^{*}=W_{\mathcal{L}^{1}}, h=0$ and $m=72 b \varepsilon^{-1} n(1-\lambda)^{-1}$.

Note that $\left\|X_{t+1}\right\|_{1}-\left\|X_{t}\right\|_{1}$ is equal to +1 if the event at time $t$ is an arrival, with probability $\lambda /(1+\lambda)$, and equal to -1 if the event is a potential departure from a non-empty queue, with probability $u_{1}\left(X_{t}\right) /(1+\lambda)$, so the drift $\Delta\|x\|_{1}$ is equal to $\frac{1}{1+\lambda}\left(\lambda-u_{1}(x)\right)$. For $x \in \mathcal{L}_{1}^{1}$, we have

$$
1-u_{1}(x)=\frac{Q_{1}(x)}{n} \leq(1-\lambda)\left(1-\frac{\varepsilon}{6}-\frac{\varepsilon}{6 k}+\frac{\varepsilon}{24 k}\right) \leq(1-\lambda)\left(1-\frac{\varepsilon}{6}\right) .
$$

Hence, for $x \in \mathcal{L}_{1}^{1},(1+\lambda) \Delta\|x\|_{1}=\left(1-u_{1}(x)\right)-(1-\lambda) \leq-\frac{\varepsilon}{6}(1-\lambda)$, and so $\Delta\|x\|_{1} \leq$ $-\frac{\varepsilon}{12}(1-\lambda):=-v$. Note that $v m=2 c$. 
Hence we may apply Lemma 3.3(i). With $T_{0}$ and $T_{1}$ as in that lemma, we have $T_{0}=W_{\mathcal{L}^{1}}^{\dagger}$ and $T_{1}=W_{\mathcal{M}}$, so we conclude that

$$
\begin{aligned}
\mathbb{P}\left(W_{\mathcal{L}^{1}}^{\dagger} \wedge W_{\mathcal{M}} \geq W_{\mathcal{L}^{1}}+m\right) & \leq \exp \left(-v^{2} m / 8\right) \\
& =\exp (-\varepsilon b n(1-\lambda) / 16) \leq 1 / 12 s_{0},
\end{aligned}
$$

as required.

We now combine Lemmas 8.2, 8.3 and 8.4 to prove Proposition 8.1.

Observe that, for a copy $\left(X_{t}\right)$ of the $(n, d, \lambda)$-supermarket process starting in a state $x_{0} \in \mathcal{L}_{1}^{k+1}$, exactly one of the following occurs:

(a) $W_{\mathcal{L}^{k+1}}^{\dagger}<m_{\mathcal{E}}$,

(b) not (a), and one of $W_{\mathcal{L}^{k}}^{\dagger}, W_{\mathcal{L}^{k-1}}^{\dagger}, \ldots, W_{\mathcal{L}^{1}}^{\dagger}$ is less than $m_{\mathcal{E}}$,

(c) neither of the above, and $W_{\mathcal{L}^{k}}>12 k \varepsilon^{-1} n(1-\lambda)^{-1}$,

(d) none of the above, and $W_{\mathcal{L}^{j}}>W_{\mathcal{L}^{j+1}}+\varepsilon^{-1} n(1-\lambda)^{-1}$ for some $j=k-1, \ldots, 1$,

(e) none of the above, and $W_{\mathcal{M}}>W_{\mathcal{L}^{1}}+72 b \varepsilon^{-1} n(1-\lambda)^{-1}$,

(f) none of the above, and $W_{\mathcal{M}}<m_{\mathcal{E}} \leq W_{\mathcal{L}^{k+1}}^{\dagger}$.

Indeed, if none of (a)-(e) occurs, then $W_{\mathcal{L}^{k+1}}^{\dagger} \geq m_{\mathcal{E}}$ since (a) fails, and also

$$
\begin{aligned}
W_{\mathcal{M}} & =W_{\mathcal{L}^{k}}+\sum_{j=1}^{k-1}\left(W_{\mathcal{L}^{j}}-W_{\mathcal{L}^{j+1}}\right)+\left(W_{\mathcal{M}}-W_{\mathcal{L}^{1}}\right) \\
& \leq 12 k \varepsilon^{-1} n(1-\lambda)^{-1}+(k-1) \varepsilon^{-1} n(1-\lambda)^{-1}+72 b \varepsilon^{-1} n(1-\lambda)^{-1} \\
& <(13 k+72 b) \varepsilon^{-1} n(1-\lambda)^{-1}=m_{\mathcal{E}} .
\end{aligned}
$$

We now show that the probability of each of (b)-(f) is small. For (b), Lemmas 8.2(2) and 8.3(2) give that

$$
\begin{aligned}
& \mathbb{P}\left(W_{\mathcal{L}^{k}}^{\dagger} \wedge W_{\mathcal{L}^{k-1}}^{\dagger} \wedge \cdots \wedge W_{\mathcal{L}^{1}}^{\dagger}<m_{\mathcal{E}} \leq W_{\mathcal{L}^{k+1}}^{\dagger}\right) \\
& \quad \leq \mathbb{P}\left(W_{\mathcal{L}^{k}}^{\dagger}<m_{\mathcal{E}} \leq W_{\mathcal{L}^{k+1}}^{\dagger}\right)+\sum_{j=1}^{k-1} \mathbb{P}\left(W_{\mathcal{L}^{j}}^{\dagger}<m_{\mathcal{E}} \leq W_{\mathcal{L}^{j+1}}^{\dagger}\right) \\
& \quad \leq \frac{1}{6 s_{0}}+(k-1) \frac{1}{3 k s_{0}} \leq \frac{1}{2 s_{0}}
\end{aligned}
$$

i.e., the probability of (b) is at most $1 / 2 s_{0}$. The probability of (c) is at most $1 / 12 s_{0}$ by Lemma 8.2(1). The probability of (d) is at most $(k-1) \frac{1}{3 k s} \leq 1 / 3 s_{0}$ by Lemma $8.3(1)$. The probability of (e) is at most $1 / 12 s_{0}$ by Lemma 8.4 . Finally, (f) is not possible, since at time $W_{\mathcal{M}}$ there are no customers in the system, so $Q_{k}\left(X_{W_{\mathcal{M}}}\right)>n$, and thus $W_{\mathcal{M}} \geq T_{\mathcal{B}}^{\dagger}$, but also $T_{\mathcal{B}}^{\dagger} \geq W_{\mathcal{L}^{k+1}}^{\dagger}$ since $\mathcal{L}_{1}^{k+1} \subseteq \mathcal{D}_{1} \subseteq \mathcal{B}_{1}$ by definition.

Thus the probability of (a), for a copy of the process starting in a state in $\mathcal{L}_{1}^{k+1}$, is at least $1-\frac{1}{2 s_{0}}-\frac{1}{12 s_{0}}-\frac{1}{3 s_{0}}-\frac{1}{12 s_{0}}=1-\frac{1}{s_{0}}$, which is what we need to prove Proposition 8.1, and thus also Lemma 6.5(1).

Now we move to the proof of Lemma $6.5(2)$, stating that the exit time $T_{\mathcal{E}}^{\dagger}$ is large with high probability. There are two things to prove here. The first is that, if $X_{t} \in \mathcal{E}_{1}$, then it is very unlikely that, at time $t+1$, a customer arrives and creates a queue of length $k+1$. The second is that, once $Q_{k}\left(X_{t}\right)$ has reached $(1-3 \varepsilon) n(1-\lambda)(\lambda d)^{k-1}$, while $u_{k+1}\left(X_{t}\right)$ is at most $\varepsilon(1-\lambda), Q_{k}$ is unlikely to "cross down against the drift" to $(1-4 \varepsilon) n(1-\lambda)(\lambda d)^{k-1}$. 
For $t \geq 0$, let $L_{t}$ denote the event that, at time $t$, a customer arrives and joins a queue of length at least $k$ (equivalently, the probability that the event is an arrival and that all the selected queues have length at least $k)$. So $L_{t}$ is the event that $u_{j}\left(X_{t}\right)>u_{j}\left(X_{t-1}\right)$ for some $j \geq k+1$.

Lemma 8.5 On the event that $X_{t} \in \mathcal{E}_{1}$, we have $\mathbb{P}\left(L_{t+1} \mid \mathcal{F}_{t}\right)<e^{-\log ^{2} n}$.

Proof From the definition of $L_{t}$, we have $\mathbb{P}\left(L_{t+1} \mid \mathcal{F}_{t}\right)=\frac{\lambda}{1+\lambda} u_{k}\left(X_{t}\right)^{d} \leq u_{k}\left(X_{t}\right)^{d}$. For $x \in \mathcal{E}_{1}$, we have $Q_{k}(x) \geq(1-4 \varepsilon) n(1-\lambda)(\lambda d)^{k-1}$ and $Q_{k-1}(x) \leq(1+5 \varepsilon) n(1-$ $\lambda)(\lambda d)^{k-2} \leq \frac{1}{3} \varepsilon n(1-\lambda)(\lambda d)^{k-1}$. Therefore, by (5.5), we have

$$
1-u_{k}(x) \geq \frac{Q_{k}(x)}{n}-\frac{Q_{k-1}(x)}{n} \geq\left(1-\frac{13}{3} \varepsilon\right)(1-\lambda)(\lambda d)^{k-1} \geq \frac{1}{2}(1-\lambda) d^{k-1} .
$$

Hence, on the event that $X_{t} \in \mathcal{E}_{1}$,

$$
u_{k}\left(X_{t}\right)^{d} \leq\left(1-\frac{1}{2}(1-\lambda) d^{k-1}\right)^{d} \leq \exp \left(-\frac{1}{2}(1-\lambda) d^{k}\right) \leq \exp \left(-\log ^{2} n\right),
$$

as required.

Let $U^{\dagger}=\inf \left\{t>T_{\mathcal{E}}: u_{k+1}\left(X_{t}\right)>\varepsilon(1-\lambda)\right\}$ and $V^{\dagger}=\inf \left\{t>T_{\mathcal{E}}: Q_{k}\left(X_{t}\right)<\right.$ $\left.(1-4 \varepsilon) n(1-\lambda)(\lambda d)^{k-1}\right\}$, and note that $T_{\mathcal{E}}^{\dagger}=T_{\mathcal{D}}^{\dagger} \wedge U^{\dagger} \wedge V^{\dagger}$. We thus have

$$
\mathbb{P}\left(T_{\mathcal{E}}^{\dagger} \leq s_{0}<T_{\mathcal{D}}^{\dagger}\right) \leq \mathbb{P}\left(U^{\dagger} \leq s_{0} \wedge T_{\mathcal{D}}^{\dagger} \wedge V^{\dagger}\right)+\mathbb{P}\left(V^{\dagger} \leq s_{0} \wedge T_{\mathcal{D}}^{\dagger} \wedge U^{\dagger}\right)
$$

We claim that each of these last two probabilities is at most $1 / 2 s_{0}$. For the first, we may apply Lemma 8.5. Observe that, if $U^{\dagger}=t+1$, then the event $L_{t+1}$ occurs. We now have:

$$
\begin{aligned}
\mathbb{P}\left(U^{\dagger} \leq s_{0} \wedge T_{\mathcal{D}}^{\dagger} \wedge V^{\dagger}\right) & =\sum_{t=0}^{s_{0}-1} \mathbb{P}\left(U^{\dagger}=t+1 \leq T_{\mathcal{D}}^{\dagger} \wedge V^{\dagger}\right) \\
=\sum_{t=0}^{s_{0}-1} \mathbb{P}\left(U^{\dagger}=t+1 \text { and } X_{t} \in \mathcal{E}_{1}\right) & =\sum_{t=0}^{s_{0}-1} \mathbb{E}\left[\mathbb{1}_{\left\{X_{t} \in \mathcal{E}_{1}\right\}} \mathbb{E}\left(\mathbb{1}_{\left\{U^{\dagger}=t+1\right\}} \mid \mathcal{F}_{t}\right)\right] \\
& \leq \sum_{t=0}^{s_{0}-1} \mathbb{E}\left[\mathbb{1}_{\left\{X_{t} \in \mathcal{E}_{1}\right\}} \mathbb{E}\left(\mathbb{1}_{L_{t+1}} \mid \mathcal{F}_{t}\right)\right] .
\end{aligned}
$$

By Lemma 8.5 , each term is at most $e^{-\log ^{2} n}$, and so we have

$$
\mathbb{P}\left(U^{\dagger} \leq s_{0} \wedge T_{\mathcal{D}}^{\dagger} \wedge V^{\dagger}\right) \leq s_{0} e^{-\log ^{2} n}<1 / 2 s_{0},
$$

as claimed.

To obtain the other required inequality, we apply the reversed version of Lemma 3.3(ii). We consider the process $\left(X_{t}\right)$, with its natural filtration, the function $F=Q_{k}$, and the set $\mathcal{S}=\left\{x: u_{k+1}(x) \leq \varepsilon(1-\lambda)\right\} \cap \mathcal{D}_{1}$. We set $h=(1-3 \varepsilon) n(1-\lambda)(\lambda d)^{k-1}$ and $\rho=\varepsilon n(1-\lambda)(\lambda d)^{k-1} \geq 2$. We also set $s=s_{0}$ and $T^{*}=T_{\mathcal{E}}$. We have $T_{0}=\inf \left\{t \geq T_{\mathcal{E}}\right.$ : $X_{t} \notin \mathcal{D}_{1}$ or $\left.u_{k+1}\left(X_{t}\right)>\varepsilon(1-\lambda)\right\}$, so that $T_{0} \geq T_{\mathcal{D}}^{\dagger} \wedge U^{\dagger}$ (strict inequality occurs if $T_{\mathcal{D}}^{\dagger}<T_{\mathcal{E}}$ ). Also $T_{1}=\inf \left\{t \geq T_{\mathcal{E}}: Q_{k}\left(X_{t}\right) \geq h\right\}=T_{\mathcal{E}}$, and $T_{2}=\inf \left\{t>T_{\mathcal{E}}: Q_{k}\left(X_{t}\right) \leq h-\rho\right\}=V^{\dagger}$.

Take $x \in \mathcal{S}$ with $Q_{k}(x) \leq h$. As $x \in \mathcal{D}_{1}$, we apply Lemma 7.1 to obtain 


$$
\begin{aligned}
(1+\lambda) \Delta Q_{k}(x) & \geq \beta_{k}\left(1-\lambda-u_{k+1}(x)\right)-\frac{Q_{k}(x)}{n(\lambda d)^{k-1}}-\frac{\varepsilon}{6}(1-\lambda) \\
& \geq \beta_{k}(1-\lambda)(1-\varepsilon)-(1-\lambda)(1-3 \varepsilon)-\frac{\varepsilon}{6}(1-\lambda) \\
& \geq(1-\lambda)\left[\left(1-\frac{\varepsilon}{2}\right)(1-\varepsilon)-1+3 \varepsilon-\frac{\varepsilon}{6}\right] \\
& \geq \varepsilon(1-\lambda) .
\end{aligned}
$$

This yields $\Delta Q_{k}(x) \geq \frac{1}{2} \varepsilon(1-\lambda):=v$, for $\operatorname{such} x$.

The reversed version of Lemma 3.3(ii) gives that

$$
\begin{aligned}
\mathbb{P}\left(V^{\dagger} \leq s_{0} \wedge T_{\mathcal{D}}^{\dagger} \wedge U^{\dagger}\right) & \leq \mathbb{P}\left(T_{2} \leq s_{0} \wedge T_{0}\right) \\
& \leq \frac{100 s_{0}}{v^{2}} \exp (-\rho v / 8) \\
& =\frac{100 s_{0}}{v^{2}} \exp \left(-\varepsilon^{2} n(1-\lambda)^{2}(\lambda d)^{k-1} / 16\right) \\
& \leq 1 / 2 s_{0},
\end{aligned}
$$

as required. This completes the proof of Lemma 6.5.

\section{Proofs of Lemmas 6.6 and 6.7}

In this section, we prove the final two of our sequence of lemmas.

\section{Proof of Lemma 6.6}

Proof Fix $j$ with $1 \leq j \leq k-1$, and consider the state of the process at the hitting time $T_{\mathcal{G}^{j+1}}$. The hitting time $T_{\mathcal{G}^{j}}$ is the first time $t \geq T_{\mathcal{G}^{j+1}}$ that $Q_{j}\left(X_{t}\right)$ lies in the interval between $\left[1-\left(4+\frac{k-j-1 / 2}{k}\right) \varepsilon\right] n(1-\lambda)(\lambda d)^{j-1}$ and $\left[1+\left(4+\frac{k-j-1 / 2}{k}\right) \varepsilon\right] n(1-\lambda)(\lambda d)^{j-1}$. Let $B_{h}$ be the event that $Q_{j}\left(X_{T_{\mathcal{G}^{j+1}}}\right)>\left[1+\left(4+\frac{k-j-1 / 2}{k}\right) \varepsilon\right] n(1-\lambda)(\lambda d)^{j-1}$, and $B_{\ell}$ be the event that $Q_{j}\left(X_{T_{\mathcal{G}} j+1}\right)<\left[1-\left(4+\frac{k-j-1 / 2}{k}\right) \varepsilon\right] n(1-\lambda)(\lambda d)^{j-1}$.

For part (1) of the lemma, we have to show that, on the event $B_{h}$, with high probability $Q_{j}\left(X_{t}\right)$ enters the interval from above within time $m_{\mathcal{G}}$, and also that, on the event $B_{\ell}$, with high probability $Q_{j}\left(X_{t}\right)$ enters the interval from below within time $m_{\mathcal{G}}$. These two results are essentially the same, and we give details only for the first. Of course, we have nothing to prove on the event that $Q_{j}\left(X_{T_{\mathcal{G}^{j+1}}}\right)$ is already in the interval.

We apply Lemma 3.3(i) to $\left(X_{t}\right)$, with its natural filtration, and the scaled function $F(x)=$ $Q_{j}^{\prime}(x)=Q_{j}(x) /(\lambda d)^{(j-1) / 2}$. We take $\mathcal{S}=\mathcal{G}_{1}^{j+1}$ and $T^{*}=T_{\mathcal{G}^{j+1}}$. We set

$$
h=\left[1+\left(4+\frac{k-j-1 / 2}{k}\right) \varepsilon\right] n(1-\lambda)(\lambda d)^{(j-1) / 2},
$$

and $m=m_{\mathcal{G}}=32 k \varepsilon^{-1} n(1-\lambda)^{-1}(\lambda d)^{-1}$. From (5.3), we have that $Q_{j}^{\prime}(x) \leq c:=2 n$ for all $x$. Also $T_{0}=T_{\mathcal{G}^{j+1}}^{\dagger}$ and $T_{1}=\inf \left\{t \geq T_{\mathcal{G}^{j+1}}: Q_{j}^{\prime}\left(X_{t}\right) \leq h\right\}$.

For $x \in \mathcal{G}_{1}^{j+1}$, we have

$$
Q_{j+1}(x) \leq\left[1+\left(4+\frac{k-j-1}{k}\right) \varepsilon\right] n(1-\lambda)(\lambda d)^{j} .
$$


(This follows from the specification of $\mathcal{G}_{1}^{j+1}$ for $j<k-1$, and since $\mathcal{G}_{1}^{k}=\mathcal{E}_{1} \subseteq \mathcal{B}_{1}$ for $j=k-1$.) If also $Q_{j}^{\prime}(x) \geq h$, we have

$$
Q_{j}(x) \geq\left[1+\left(4+\frac{k-j-1 / 2}{k}\right) \varepsilon\right] n(1-\lambda)(\lambda d)^{j-1} .
$$

Lemma 7.1 applies since $x \in \mathcal{D}_{1}$, so

$$
\begin{aligned}
(1+\lambda) \Delta Q_{j}(x) \leq & -\lambda d \frac{Q_{j}(x)}{n}\left(1-\frac{\varepsilon}{25 k}\right)+\frac{Q_{j+1}(x)}{n} \\
\leq & -\left[1+\left(4+\frac{k-j-1 / 2}{k}\right) \varepsilon\right](1-\lambda)(\lambda d)^{j}\left(1-\frac{\varepsilon}{25 k}\right) \\
& +\left[1+\left(4+\frac{k-j-1}{k}\right) \varepsilon\right](1-\lambda)(\lambda d)^{j} \\
\leq & -\frac{1}{4 k} \varepsilon(1-\lambda)(\lambda d)^{j},
\end{aligned}
$$

and so $\Delta Q_{j}^{\prime}(x) \leq-\frac{1}{8 k} \varepsilon(1-\lambda)(\lambda d)^{(j+1) / 2}:=-v$. Note that $v m_{\mathcal{G}} \geq 2 c$.

Lemma 3.3(i) now gives

$$
\begin{aligned}
\mathbb{P}\left(T_{1} \wedge T_{0}>T_{\mathcal{G}^{j+1}}+m_{\mathcal{G}}\right) & \leq \exp \left(-v^{2} m_{\mathcal{G}} / 8\right) \\
& =\exp \left(-\varepsilon n(1-\lambda)(\lambda d)^{(j+1) / 2} / 16 k\right) \\
& \leq 1 / 2 s_{0} .
\end{aligned}
$$

On the $T_{\mathcal{G}^{j}}$-measurable event $B_{h}$, the stopping times $T_{1} \wedge T_{0}$ and $T_{\mathcal{G}^{j}} \wedge T_{0}$ coincide, so we have

$$
\mathbb{P}\left(B_{h} \cap\left\{T_{\mathcal{G}^{j}} \wedge T_{\mathcal{G}^{j+1}}^{\dagger}>T_{\mathcal{G}^{j+1}}+m_{\mathcal{G}}\right\}\right) \leq 1 / 2 s_{0}
$$

Essentially exactly the same calculation gives

$$
\mathbb{P}\left(B_{\ell} \cap\left\{T_{\mathcal{G}^{j}} \wedge T_{\mathcal{G}^{j+1}}^{\dagger}>T_{\mathcal{G}^{j+1}}+m_{\mathcal{G}}\right\}\right) \leq 1 / 2 s_{0},
$$

and part (1) of the lemma now follows, for this value of $j$.

To prove part (2) of the lemma, we need to show that, once $X_{t}$ has reached $\mathcal{G}_{0}^{j}$, and while it remains in $\mathcal{G}_{1}^{j+1}$, the process is unlikely to leave the set $\mathcal{G}_{1}^{j}$ quickly. There are two separate things to prove: that $Q_{j}\left(X_{t}\right)$ is unlikely to cross against the drift from $[1+(4+$ $\left.\left.\frac{k-j-1 / 2}{k}\right) \varepsilon\right] n(1-\lambda)(\lambda d)^{j-1}$ to $\left[1+\left(4+\frac{k-j}{k}\right) \varepsilon\right] n(1-\lambda)(\lambda d)^{j-1}$ before time $s_{0}$, and also that $Q_{j}\left(X_{t}\right)$ is unlikely to cross against the drift from $\left[1-\left(4+\frac{k-j-1 / 2}{k}\right) \varepsilon\right] n(1-\lambda)(\lambda d)^{j-1}$ to $\left[1-\left(4+\frac{k-j}{k}\right) \varepsilon\right] n(1-\lambda)(\lambda d)^{j-1}$ before time $s_{0}$. Again, the two calculations required here are essentially identical, and we shall concentrate on the first.

We apply Lemma 3.3(ii), again for the process $\left(X_{t}\right)$ with its natural filtration, and the scaled function $F(x)=Q_{j}(x) /(\lambda d)^{(j-1) / 2}$. We take the same values of parameters as above, and additionally set $\rho=\frac{\varepsilon}{2 k} n(1-\lambda)(\lambda d)^{(j-1) / 2}$ and $s=s_{0}$. As before, we may take $p=2(1-\lambda) d^{j}$. Here $T_{2}=\inf \left\{t>T_{1}: Q_{j}^{\prime}\left(X_{t}\right) \geq h+\rho\right\}$. 


$$
\begin{aligned}
\mathbb{P}\left(T_{2} \leq s_{0}<T_{\mathcal{G}^{j+1}}^{\dagger}\right) & \leq \frac{100 s_{0}}{v^{2}} \exp (-\rho v / 8 p) \\
& =\frac{100 s_{0}}{v^{2}} \exp \left(-\varepsilon^{2} n(1-\lambda) \lambda^{j} / 256 k\right) \\
& \leq 1 / 2 s_{0} .
\end{aligned}
$$

Setting $U_{2}=\inf \left\{t>T_{1}: Q_{j}\left(X_{t}\right) \leq\left[1-\left(4+\frac{k-j}{k}\right) \varepsilon\right] n(1-\lambda)(\lambda d)^{j-1}\right\}$, we have, similarly, $\mathbb{P}\left(U_{2} \leq s_{0}<T_{\mathcal{G}^{j+1}}^{\dagger}\right) \leq 1 / 2 s_{0}$.

The events $T_{2} \wedge U_{2} \leq s_{0}<T_{\mathcal{G}^{j+1}}^{\dagger}$ and $T_{\mathcal{G}^{j}}^{\dagger} \leq s_{0}<T_{\mathcal{G}^{j+1}}^{\dagger}$ coincide, so

$$
\begin{aligned}
\mathbb{P}\left(T_{\mathcal{G}^{j}}^{\dagger} \leq s_{0}<T_{\mathcal{G}^{j+1}}^{\dagger}\right) & \leq \mathbb{P}\left(T_{2} \leq s<T_{0}\right)+\mathbb{P}\left(U_{2} \leq s<T_{0}\right) \\
& \leq \frac{1}{2 s_{0}}+\frac{1}{2 s_{0}}=\frac{1}{s_{0}},
\end{aligned}
$$

as required for part (2) for this value of $j$.

\section{Proof of Lemma 6.7}

Proof We first prove part (1). For $i=1, \ldots, n$, let $N_{i}$ be the number of potential departures from queue $i$ over the time period between $T_{\mathcal{G}^{1}}$ and $T_{\mathcal{G}^{1}}+m_{\mathcal{H}}$, so $N_{i}$ is a binomial random variable with parameters $\left(m_{\mathcal{H}}, 1 / n(1+\lambda)\right)$. Recall that $L_{t}$ is the event that, at time $t$, a customer arrives and joins a queue of length $k$ or longer, and observe that

$$
\mathbb{P}\left(T_{\mathcal{H}} \wedge T_{\mathcal{G}^{1}}^{\dagger} \geq T_{\mathcal{G}^{1}}+m_{\mathcal{H}}\right) \leq \mathbb{P}\left(\bigcup_{t=T_{\mathcal{G}^{1}}+1}^{T_{\mathcal{G}^{1}}+m_{\mathcal{H}}}\left(L_{t} \cap\left\{X_{t-1} \in \mathcal{G}_{1}^{1}\right\}\right)\right)+\mathbb{P}\left(\exists i, N_{i}<3 \ell\right) .
$$

Indeed, at time $T_{\mathcal{G}^{1}}$, the process is in $\mathcal{A}_{1}(\ell, g)$, and so there is no queue with more than $3 \ell$ customers in it at that time. If there are at least $3 \ell$ potential departures from each queue over the time interval, and $\bigcup_{t=T_{\mathcal{G}}{ }^{1}+1}^{T_{\mathcal{G}}+m_{\mathcal{H}}} L_{t}$ does not occur, then by time $T_{\mathcal{G}^{1}}+m_{\mathcal{H}}$, every queue is reduced to length at most $k$, and no new queue of length $k+1$ is created before $T_{\mathcal{G}^{1}}+m_{\mathcal{H}}$.

Now let $\left(X_{t}^{\prime}\right)=\left(X_{T_{\mathcal{G}^{1}}+t}\right),\left(\mathcal{F}_{t}^{\prime}\right)=\left(\mathcal{F}_{T_{\mathcal{G}^{1}}+t}\right)$ and $L_{t}^{\prime}=L_{T_{\mathcal{G}^{1}}+t}$. We have:

$$
\begin{aligned}
\mathbb{P}\left(\bigcup_{t=T_{\mathcal{G}^{1}}+1}^{T_{\mathcal{G}^{1}}+m_{\mathcal{H}}}\left(L_{t} \cap\left\{X_{t-1} \in \mathcal{G}_{1}^{1}\right\}\right)\right) & =\mathbb{P}\left(\bigcup_{t=1}^{m_{\mathcal{H}}}\left(L_{t}^{\prime} \cap\left\{X_{t-1}^{\prime} \in \mathcal{G}_{1}^{1}\right\}\right)\right) \\
& \leq \sum_{t=1}^{m_{\mathcal{H}}} \mathbb{P}\left(L_{t}^{\prime} \cap\left\{X_{t-1}^{\prime} \in \mathcal{G}_{1}^{1}\right\}\right) \\
& =\sum_{t=1}^{m_{\mathcal{H}}} \mathbb{E}\left[\mathbb{1}_{\left\{X_{t-1}^{\prime} \in \mathcal{G}_{1}^{1}\right\}} \mathbb{E}\left[\mathbb{1}_{L_{t}^{\prime}} \mid \mathcal{F}_{t-1}^{\prime}\right]\right] \\
& \leq m_{\mathcal{H}} e^{-\log ^{2} n} \leq 1 / 2 s_{0},
\end{aligned}
$$

where we used the strong Markov property, and Lemma 8.5.

Recall that $m_{\mathcal{H}}=n\left(8 \ell+32 \log ^{2} n\right)$, so that the mean $\mu$ of each $N_{i}$ is $m_{\mathcal{H}} / n(1+\lambda) \geq$ $4 \ell+16 \log ^{2} n$. By (3.1), with $\varepsilon=1 / 4$, we have

$$
\mathbb{P}\left(N_{i} \leq 3 \ell\right) \leq \mathbb{P}\left(N_{i} \leq \frac{3}{4} \mu\right) \leq e^{-\mu / 32} \leq e^{-\frac{1}{2} \log ^{2} n}
$$


for each $i$. Thus the probability that there are fewer than $3 \ell$ departures from any queue over the interval from $T_{\mathcal{G}^{1}}$ to $T_{\mathcal{G}^{1}}+m_{\mathcal{H}}$ is at most $n e^{-\frac{1}{2} \log ^{2} n}<1 / 2 s_{0}$, and part (1) follows.

For part (2), as above we have

$$
\mathbb{P}\left(\bigcup_{t=T_{\mathcal{G}^{1}}+1}^{T_{\mathcal{G}^{1}}+s_{0}}\left(L_{t} \cap\left\{X_{t-1} \in \mathcal{G}_{1}^{1}\right\}\right)\right) \leq s_{0} e^{-\log ^{2} n} \leq 1 / s_{0} .
$$

Thus $\mathbb{P}\left(T_{\mathcal{H}}^{\dagger} \leq s_{0}<T_{\mathcal{G}_{1}}^{\dagger}\right)$ is at most the probability that $X_{t}$ exits the set $\mathcal{H}_{1}$ before time $T_{\mathcal{G}_{1}}^{\dagger} \wedge s_{0}$, necessarily by the creation of a new queue of length $k+1$, is at most $1 / s_{0}$, as required.

\section{Rapid Mixing}

In this section, we prove our results about rapid mixing of the $(n, d, \lambda)$-supermarket process. We continue to assume that the functions $\lambda=\lambda(n)=1-n^{-\alpha}, d=d(n)=n^{\beta}$ and $\varepsilon=\varepsilon(n)$ of the model satisfy the conditions of Theorem 6.1. We also assume throughout this section that $b \leq \ell \leq e^{\frac{1}{4} \log ^{2} n}$, so that $q(\ell, b)=(22 k+72 b) \varepsilon^{-1} n^{1+\alpha}+8 \ell n \leq s_{0} / 2$.

We say that two queue-lengths vectors are adjacent if they differ by one customer in one queue, and we first consider two copies of the process starting in adjacent states in $\mathcal{A}_{0}(\ell, b)$, coupled according to the coupling referred to in Lemma 4.1. The proof partly follows along the lines of the proof of Lemma 2.6 in [11].

Lemma 10.1 Let $x, y$ be a pair of adjacent states in $\mathcal{A}_{0}(\ell, b)$, with $x\left(j_{0}\right)=y\left(j_{0}\right)-1$ for some queue $j_{0}$, and $x(j)=y(j)$ for $j \neq j_{0}$. Consider coupled copies $\left(X_{t}^{x}\right)$ and $\left(X_{t}^{y}\right)$ of the $(n, d, \lambda)$-supermarket process, where $X_{0}^{x}=x$ and $X_{0}^{y}=y$. For $n$ sufficiently large, and all times $t \geq 2 q(\ell, b)$, we have

$$
\mathbb{E}\left\|X_{t}^{x}-X_{t}^{y}\right\|_{1}=\mathbb{P}\left(X_{t}^{x} \neq X_{t}^{y}\right) \leq 2 e^{-\frac{1}{4} \log ^{2} n} .
$$

Proof By Lemma 4.1, $X_{t}^{x}$ and $X_{t}^{y}$ are always neighbours or equal, always $X_{t}^{x} \leq X_{t}^{y}$, and if for some time $s$ we have $X_{s}^{x}=X_{s}^{y}$, then $X_{t}^{x}=X_{t}^{y}$ for all $t \geq s$. Thus in particular $\mathbb{E}\left\|X_{t}^{x}-X_{t}^{y}\right\|_{1}=\mathbb{P}\left(X_{t}^{x} \neq X_{t}^{y}\right)$. The probability of coalescence is increasing with $t$, so we may assume that $t=2 q(\ell, b)$.

Initially, the queue $j_{0}$ is unbalanced, i.e., $X_{0}^{x}\left(j_{0}\right) \neq X_{0}^{y}\left(j_{0}\right)$, and all other queues are balanced. Observe that the index of the unbalanced queue in the coupled pair of processes may change over time. Let $W_{t}$ denote the longer of the unbalanced queue lengths at time $t$, if there is such a queue, and let $W_{t}=0$ otherwise. The time for the two coupled processes to coalesce is the time $T$ until $W_{t}$ hits 0 .

We first run $\left(X_{t}^{x}\right)$ and $\left(X_{t}^{y}\right)$ together using the coupling. Let $T_{\mathcal{H}}^{x}$ and $T_{\mathcal{H}}^{y}$ denote the times $T_{\mathcal{H}}$, as defined in Sect. 6, for the two copies of the process, and set $T_{\mathcal{H}}^{*}=T_{\mathcal{H}}^{x} \vee T_{\mathcal{H}}^{y}$. By Theorem $6.10, T_{\mathcal{H}}^{*} \leq q(\ell, b)$ with probability at least

$$
1-\frac{2(6 k+28)}{s_{0}} \geq 1-\frac{1}{3} e^{-\frac{1}{4} \log ^{2} n}
$$

We now track the performance of the coupling after time $T_{\mathcal{H}}^{*}$. If the processes have coalesced by time $T_{\mathcal{H}}^{*}$ (i.e., if $T \leq T_{\mathcal{H}}^{*}$ ), then we are done. Otherwise, $X_{T_{\mathcal{H}}^{*}}^{x}$ and $X_{T_{\mathcal{H}}^{*}}^{y}$ are still adjacent, and there is some random index $J_{0}$ such that the queue $J_{0}$ is unbalanced, i.e., 
$X_{T_{\mathcal{H}}^{*}}^{x}\left(J_{0}\right) \neq X_{T_{\mathcal{H}}^{*}}^{y}\left(J_{0}\right)$, and all other queues are balanced. Moreover, since $u_{k+1}(x)=0$ for all $x \in \mathcal{H}$, we have $W_{T_{\mathcal{H}}^{*}} \leq k$.

We shall use Lemma 3.4 to give a suitable upper bound on $\mathbb{P}\left(W_{t}>0\right)$. The idea is that, since, with high probability, both copies of the process remain in $\mathcal{H}$ for a long time, the unbalanced queue length $W_{t}$ will often drop below $k$, and then there is a chance of going all the way down to 0 before returning to $k$.

For each $t \geq 0$, let $B_{t}$ be the event that $X_{s}^{y}, X_{s}^{x} \in \mathcal{H}$ for all $s$ with $T_{\mathcal{H}}^{*} \leq s \leq t-1$. It follows from Theorem 6.10 that $\mathbb{P}\left(\bar{B}_{t}\right) \leq(12 k+56) / s_{0} \leq \frac{1}{3} e^{-\frac{1}{4} \log ^{2} n}$, provided $t \leq s_{0}$.

Let $N_{r}$ be the number of jumps of the longer unbalanced queue length in the first $r$ steps after $T_{\mathcal{H}}^{*}$. Also set $N=N_{T}$, the total number of these jumps, with $N_{T}=0$ if $T \leq T_{\mathcal{H}}^{*}$. For $j=1,2, \ldots$, let $T_{j}$ be the time of the $j$ th jump after $T_{\mathcal{H}}^{*}$ if $N \geq j$, and otherwise set $T_{j}=T_{\mathcal{H}}^{*} \vee T$. Thus, if $T_{\mathcal{H}}^{*}<T$, we have $T_{\mathcal{H}}^{*}<T_{1}<\cdots<T=T_{N}=T_{N+1}=\cdots$. If $T_{\mathcal{H}}^{*} \geq T$, then all of the $T_{j}$ are equal to $T_{\mathcal{H}}^{*}$.

Let $S_{0}=y\left(J_{0}\right) \mathbb{1}_{\left\{T_{\mathcal{H}}^{*}<T\right\}}=W_{T_{\mathcal{H}}^{*}} \mathbb{1}_{\left\{T_{\mathcal{H}}<T\right\}}$, the longer unbalanced queue length at time $t=T_{\mathcal{H}}^{*}$ if coalescence has not occurred. For each positive integer $j$, if $N \geq j$, let $S_{j}=W_{T_{j}}$, which is either 0 or the longer of the unbalanced queue lengths at time $T_{j}$, immediately after the $j$ th arrival or departure at the unbalanced queue. Also, if $N \geq j$, let $Z_{j}$ be the \pm 1 -valued random variable $S_{j}-S_{j-1}$. For each non-negative integer $j$, let $\varphi_{j}$ be the $\sigma$-field $\mathcal{F}_{T_{j+1}-1}$ of all events before time $T_{j+1}$. Let also $A_{j}$ be the $\varphi_{j}$-measurable event $B_{T_{j+1}}$, that is the event that $X_{s}^{y}, X_{s}^{x} \in \mathcal{H}$ for each $s$ with $T_{\mathcal{H}}^{*} \leq s \leq T_{j+1}-1$.

We shall use Lemma 3.4. We take the sequences $\left(\varphi_{j}\right)_{j \geq 0},\left(Z_{j}\right)_{j \geq 0},\left(S_{j}\right)_{j \geq 0}$ and $\left(A_{j}\right)_{j \geq 0}$ as defined above, and we set $k_{0}=k$ and $\delta=1 /(\lambda d+1)$. Note first that, at any time $t<T$, the probability, conditioned on $\mathcal{F}_{t}$, of an arrival to the longer of the unbalanced queues is at most $d \lambda / n(1+\lambda)$, while the conditional probability of a departure from that queue is $1 / n(1+\lambda)$. Therefore, on the event that $N \geq j$, the probability, conditioned on $\varphi_{j-1}$, that the event at time $T_{j}$ is a departure from the longer unbalanced queue is at least

$$
\frac{1 / n(1+\lambda)}{1 / n(1+\lambda)+d \lambda / n(1+\lambda)}=\frac{1}{1+d \lambda}=\delta .
$$

In other words, on the event $N \geq j$ we have $\mathbb{P}\left(Z_{j}=-1 \mid \varphi_{j-1}\right) \geq \delta$.

We now show that, on the event $\{N \geq j\} \cap A_{j-1} \cap\left\{S_{j-1} \geq k\right\}$, we have

$$
\mathbb{P}\left(Z_{j}=-1 \mid \varphi_{j-1}\right) \geq \frac{3}{4} .
$$

To see this, consider a time $t \geq T_{\mathcal{H}}^{*}$. On the event $B_{t}$, we have $X_{t} \in \mathcal{H} \subseteq \mathcal{E}_{1}$, and so, by Lemma 8.5 , the conditional probability $\mathbb{P}\left(L_{t+1} \mid \mathcal{F}_{t}\right)$ that the event at time $t+1$ is an arrival to a queue of length $k$ or greater is at most $e^{-\log ^{2} n}$. In particular, on the event $B_{t} \cap\left\{W_{t-1} \geq k\right\}$, the conditional probability that the event at time $t+1$ is an arrival joining the longer unbalanced queue is at most $e^{-\log ^{2} n}$, while the conditional probability that the event at time $t+1$ is a departure from the longer unbalanced queue is $1 / n(1+\lambda)$. Therefore, on the event $\{N \geq j\} \cap A_{j-1} \cap\left\{S_{j-1} \geq k\right\}$, we have

$$
\mathbb{P}\left(Z_{j}=-1 \mid \varphi_{j-1}\right) \geq \frac{1 / n(\lambda+1)}{1 / n(\lambda+1)+e^{-\log ^{2} n}} \geq \frac{3}{4} .
$$

We have now shown that $S_{m}-S_{0}$ can be written as a sum $\sum_{i=1}^{m} Z_{i}$ for $\{0, \pm 1\}$-valued random variables $Z_{i}$ that satisfy the conditions of Lemma 3.4, with $k_{0}=k$ and $\delta=1 /(\lambda d+1)$. (The argument above establishes this for $m \leq N$ : for $m>N$, we have set $Z_{m}=S_{m}=0$, 
which also meets the requirements of the lemma.) Note that $\delta^{-(k-1)}=(\lambda d+1)^{k-1} \leq 2 d^{k-1}$. Hence, for $m \geq 16 k$,

$$
\begin{aligned}
\mathbb{P}\left(\bigcap_{i=1}^{m}\left\{S_{i} \neq 0\right\} \cap \bigcap_{i=0}^{m-1} A_{i}\right) & \leq \mathbb{P}\left(S_{0}>\lfloor m / 16\rfloor\right)+3 \exp \left(-\frac{\delta^{k-1}}{200 k} m\right) \\
& \leq 0+3 \exp \left(-\frac{m}{400 k d^{k-1}}\right) .
\end{aligned}
$$

Here $\mathbb{P}(\cdot)$ refers to the coupling measure in the probability space of Sect. 4 , with coupled copies of the process for each possible starting state.

Let $q=q(\ell, b)$ and $m=\lfloor q / 4 n\rfloor \geq n^{\alpha}$. Since, at each time after $T_{\mathcal{H}}^{*}$ and before $T$, a jump in the longer unbalanced queue occurs with probability at least $1 / 2 n$ while the queue is nonempty, we have, by inequality (3.1), $\mathbb{P}\left(\left\{T>T_{\mathcal{H}}^{*}+q\right\} \cap\left\{N_{q}<m\right\}\right) \leq e^{-q / 16 n}$. Also,

$$
\mathbb{P}\left(\left\{N_{r} \geq m\right\} \cap \bigcup_{i=0}^{m-1} \overline{A_{i}} \cap\left\{T_{\mathcal{H}}^{*} \leq q\right\}\right) \leq \mathbb{P}\left(\overline{B_{2 q}}\right) \leq \mathbb{P}\left(\overline{B_{s_{0}}}\right) \leq \frac{1}{3} e^{-\frac{1}{4} \log ^{2} n}
$$

Now we have that

$$
\begin{aligned}
\mathbb{P}(T>2 q) \leq & \mathbb{P}\left(T_{\mathcal{H}}^{*}>q\right)+\mathbb{P}\left(\left\{T>T_{\mathcal{H}}^{*}+q\right\} \cap\left\{T_{\mathcal{H}}^{*} \leq q\right\}\right) \\
\leq & \mathbb{P}\left(T_{\mathcal{H}}^{*}>q\right)+\mathbb{P}\left(\left\{T>T_{\mathcal{H}}^{*}+q\right\} \cap\left\{N_{q}<m\right\}\right) \\
& +\mathbb{P}\left(\left\{N_{q} \geq m\right\} \cap \bigcup_{i=0}^{m-1} \overline{A_{i}} \cap\left\{T_{\mathcal{H}}^{*} \leq q\right\}\right) \\
& +\mathbb{P}\left(\left\{N_{q} \geq m\right\} \cap \bigcap_{i=0}^{m-1} A_{i} \cap \bigcap_{i=1}^{m}\left\{S_{i} \neq 0\right\}\right) .
\end{aligned}
$$

To see this, note that $\left\{N_{q} \geq m\right\} \cap \bigcup_{i=1}^{m}\left\{S_{i}=0\right\} \subseteq\left\{T \leq T_{\mathcal{H}}^{*}+q\right\}$. Now we have

$$
\begin{aligned}
\mathbb{P}(T>2 q) \leq & \frac{1}{3} e^{-\frac{1}{4} \log ^{2} n}+e^{-q / 16 n}+\frac{1}{3} e^{-\frac{1}{4} \log ^{2} n} \\
& +3 \exp \left(-\frac{q}{1600 k d^{k-1} n}\right) \\
\leq & \frac{2}{3} e^{-\frac{1}{4} \log ^{2} n}+4 \exp \left(-\frac{n^{\alpha-(k-1) \beta}}{1600 k}\right) \\
\leq & e^{-\frac{1}{4} \log ^{2} n}
\end{aligned}
$$

as required.

Theorem 10.2 Let $\left(X_{t}^{x}\right)$ and $\left(X_{t}^{y}\right)$ be two copies of the $(n, d, \lambda)$-supermarket process, starting in states $x$ and $y$ in $\mathcal{A}_{0}(\ell, b)$. Then, for $n$ sufficiently large and $t \geq 2 q(\ell, b)$, we have

$$
\mathbb{E}\left\|X_{t}^{x}-X_{t}^{y}\right\|_{1} \leq 2 b n e^{-\frac{1}{4} \log ^{2} n} \leq e^{-\frac{1}{5} \log ^{2} n} .
$$

Proof Given two distinct states $x$ and $y$ in $\mathcal{A}_{0}(\ell, b)$, we can choose a path $x=$ $z_{0}, z_{1}, \ldots, z_{m}=y$ of adjacent states in $\mathcal{A}_{0}(\ell, b)$ from $x$ down to the empty queue-lengths vector and back up to $y$, where $m=\|x\|_{1}+\|y\|_{1} \leq 2 b n$. By Lemma 10.1, for $t \geq 2 q(\ell, b)$, 


$$
\mathbb{E}\left\|X_{t}^{x}-X_{t}^{y}\right\|_{1} \leq \sum_{i=0}^{m-1} \mathbb{E}\left\|X_{t}^{z_{i}}-X_{t}^{z_{i+1}}\right\|_{1} \leq 2 b n e^{-\frac{1}{4} \log ^{2} n}
$$

as required.

We saw in Corollary 6.9 that $Y_{t} \in \mathcal{A}_{0}(\ell, b)$ with probability at least $1-e^{-\frac{1}{4} \log ^{2} n}$, whenever $\ell, b \geq k$, where $\left(Y_{t}\right)$ is a copy of the $(n, d, \lambda)$-supermarket process in equilibrium. Thus we have the following corollary.

Corollary 10.3 Take any $\ell, b \geq k$, and let $\left(X_{t}^{x}\right)$ be a copy of the $(n, d, \lambda)$-supermarket process starting in a state $x \in \mathcal{A}_{0}(\ell, b)$. Also let $\left(Y_{t}\right)$ be a copy in equilibrium. Then, for $n$ sufficiently large and $t \geq 2 q(\ell, b)$, we have

$$
d_{T V}\left(\mathcal{L}\left(X_{t}^{x}\right), \mathcal{L}\left(Y_{t}\right)\right) \leq 2 e^{-\frac{1}{5} \log ^{2} n} .
$$

This now implies Theorem 1.4. We choose $\varepsilon(n)=1 / 100$. The hypothesis that $\ell=$ $\|x\|_{\infty} \leq e^{\frac{1}{4} \log ^{2} n}$, together with $b=\|x\|_{1} / n \leq \ell$, ensures that $q(\ell, b) \leq \frac{1}{2} s_{0}$, and the setting of $\varepsilon$ ensures that $q(\ell, b) \leq 7200\left(k n^{1+\alpha}+b n^{1+\alpha}+\ell n\right)$.

We now show that mixing actually takes place faster if we start from a "good" state, i.e., a state in $\mathcal{N}=\mathcal{N}^{\varepsilon}$.

Lemma 10.4 Let $x, y$ be a pair of adjacent states in $\mathcal{N}^{\varepsilon}$, with $x\left(j_{0}\right)=y\left(j_{0}\right)-1$ for some queue $j_{0}$, and $x(j)=y(j)$ for $j \neq j_{0}$. Consider coupled copies $\left(X_{t}^{x}\right)$ and $\left(X_{t}^{y}\right)$ of the $(n, d, \lambda)$-supermarket process. For $n$ sufficiently large and all times $t \geq 0$, we have

$$
\mathbb{E}\left\|X_{t}^{x}-X_{t}^{y}\right\|_{1} \leq e^{-\frac{1}{4} \log ^{2} n}+4 \exp \left(-\frac{t}{1600 k d^{k-1} n}\right) .
$$

Proof The proof is nearly identical to that of Lemma 10.1. Instead of starting by running the two copies of the process together until some time $T^{*}$, we use the final part of Theorem 6.1, which tells us that, with probability at most $1-e^{-\frac{1}{4} \log ^{2} n}$, both $X_{t}^{x}$ and $X_{t}^{y}$ remain within $\mathcal{N}^{6 \varepsilon}$ throughout the interval $0 \leq t \leq s_{0}$. We may thus repeat the proof of Lemma 10.1 with $T^{*}$ and $q=q(\ell, b)$ replaced by 0 , and running the second phase for any number $t$ of steps instead of $q$, and we obtain the result.

As before, we can use this result to deduce an upper bound on the mixing time, starting from a good state.

Theorem 10.5 Let $\left(X_{t}^{x}\right)$ and $\left(X_{t}^{y}\right)$ be two copies of the $(n, d, \lambda)$-supermarket process with starting states $x$ and $y$ in $\mathcal{N}^{\varepsilon}$. Then, for $n$ sufficiently large and $t \geq 0$, we have

$$
\mathbb{E}\left\|X_{t}^{x}-X_{t}^{y}\right\|_{1} \leq n\left(e^{-\frac{1}{4} \log ^{2} n}+4 \exp \left(-\frac{t}{1600 k d^{k-1} n}\right)\right) .
$$

Proof (Sketch) Take any two queue-lengths vectors $x$ and $y$ in $\mathcal{N}^{\varepsilon}$. It is straightforward to show that there is a path between $x=z_{0} z_{1} \cdots z_{m}=y$ in $\mathcal{N}^{\varepsilon}$ of length $m \leq 4 n(1-$ $\lambda)(\lambda d)^{k-1} \leq n$ between $x$ and $y$. The result now follows as in the proof of Theorem 10.2.

As before, since $Y_{0}$ lies in $\mathcal{H}^{\varepsilon} \subseteq \mathcal{N}^{\varepsilon}$ with probability at least $1-e^{-\frac{1}{4} \log ^{2} n}$, by Corollary 6.9, we may now deduce that the total variation distance $d_{T V}\left(\mathcal{L}\left(X_{t}^{x}\right), \mathcal{L}\left(Y_{t}\right)\right)$ is at most 


$$
\begin{gathered}
e^{-\frac{1}{4} \log ^{2} n}+n\left(e^{-\frac{1}{4} \log ^{2} n}+4 \exp \left(-\frac{t}{1600 k d^{k-1} n}\right)\right) \\
\leq n\left(2 e^{-\frac{1}{4} \log ^{2} n}+4 \exp \left(-\frac{t}{1600 k n^{1+(k-1) \beta}}\right)\right)
\end{gathered}
$$

whenever $x \in \mathcal{N}^{\varepsilon}$. This result is exactly the statement of Theorem 1.2 (where we take $\varepsilon=1 / \log n$ : the result would hold if our initial state were in $\mathcal{N}^{\varepsilon}$ for $\varepsilon$ a suitably small constant).

Theorem 1.2 shows that, from states $x \in \mathcal{N}$, we have mixing to equilibrium in time of order $n^{1+(k-1) \beta} \log n$. We finish by proving Theorem 1.3 , showing that this bound is approximately best possible.

Note that there is a state $z$ in $\mathcal{I}^{\varepsilon} \subseteq \mathcal{H}^{\varepsilon} \subseteq \mathcal{N}^{\varepsilon}$ with $Q_{k}(z) \leq(1-3 \varepsilon) n(1-\lambda)(\lambda d)^{k-1}$. However, we know from Corollary 6.9 that $\mathbb{P}\left(Y_{t} \in \mathcal{H}^{\varepsilon / 5}\right) \geq 1-e^{-\frac{1}{4} \log ^{2} n}$, so in order for $d_{T V}\left(\mathcal{L}\left(X_{t}^{z}\right), \Pi\right)$ to be small, we need that $Q_{k}\left(X_{t}^{z}\right) \geq(1-\varepsilon) n(1-\lambda)(\lambda d)^{k-1}$ with high probability. Set $t=\frac{1}{6} n(\lambda d)^{k-1}$.

For $x \in \mathcal{H}^{\varepsilon}$, we obtain from Lemma 7.1, with a calculation almost exactly as in Lemma 8.2, that

$$
\begin{aligned}
(1+\lambda) \Delta Q_{k}(x) & \leq(1-\lambda)(1+\varepsilon / 6)-\frac{Q_{k}(x)}{n(\lambda d)^{k-1}}+\exp \left(-d Q_{k}(x) / k n\right) \\
& \leq(1-\lambda)(1+\varepsilon / 3-(1-4 \varepsilon)) \leq 5 \varepsilon(1-\lambda)
\end{aligned}
$$

so $\Delta Q_{k}(x) \leq 5 \varepsilon(1-\lambda)$ also. We know from (6.1) that, with probability at least $1-e^{-\frac{1}{4} \log ^{2} n}$, $X_{s}^{z} \in \mathcal{H}^{\varepsilon}$ for all $s=0, \ldots, t-1$, and we also have that $Q_{k}(x) \leq k n$ for every state $x$. It follows that

$$
\begin{aligned}
\mathbb{E} Q_{k}\left(X_{t}^{z}\right) & =Q_{k}(z)+\sum_{s=0}^{t-1} \mathbb{E}\left(\mathbb{E}\left(\Delta Q_{k}\left(X_{s}^{z}\right) \mid \mathcal{F}_{s}\right)\right) \\
& \leq(1-3 \varepsilon) n(1-\lambda)(\lambda d)^{k-1}+5 \varepsilon t(1-\lambda)+k n e^{-\frac{1}{4} \log ^{2} n} \\
& \leq(1-2 \varepsilon) n(1-\lambda)(\lambda d)^{k-1} .
\end{aligned}
$$

A result from [11] (adapted for discrete time) states that, for some absolute constant $c$, for any 1-Lipschitz function $f$, any starting state $z$, any $t>0$ and any $u \geq 0$,

$$
\mathbb{P}\left(\left|f\left(X_{t}^{z}\right)-\mathbb{E} f\left(X_{t}^{z}\right)\right| \geq u\right) \leq n e^{-c u^{2} /(t+u)} .
$$

Applying this with $f=Q_{k}, t=\frac{1}{6} n(\lambda d)^{k-1}$ and $u=\varepsilon t(1-\lambda)$, we find that

$$
\begin{aligned}
\mathbb{P}\left(Q_{k}\left(X_{t}^{z}\right)>(1-\varepsilon) n(1-\lambda)(\lambda d)^{k-1}\right) & \leq \mathbb{P}\left(Q_{k}\left(X_{t}^{z}\right)-\mathbb{E} Q_{k}\left(X_{t}^{z}\right)>\varepsilon n(1-\lambda)(\lambda d)^{k-1}\right) \\
& \leq n e^{-c \varepsilon^{2} n(1-\lambda)^{2}(\lambda d)^{k-1} / 2} \leq 1 / s_{0}
\end{aligned}
$$

This completes the proof of Theorem 1.3.

Open Access This article is distributed under the terms of the Creative Commons Attribution 4.0 International License (http://creativecommons.org/licenses/by/4.0/), which permits unrestricted use, distribution, and reproduction in any medium, provided you give appropriate credit to the original author(s) and the source, provide a link to the Creative Commons license, and indicate if changes were made. 


\section{References}

1. Alanyali, M., Dashouk, M.: Occupancy distributions of homogeneous queueing systems under opportunistic scheduling. IEEE Trans. Inf. Theor. 57, 256-266 (2011)

2. Brightwell, G., Luczak, M.: Vertices of high degree in the preferential attachment tree. Electron. J. Probab. 17(14), 1-43 (2012)

3. Brightwell, G., Luczak, M.: The supermarket model with arrival rate tending to one (2012). arXiv: 1201.5523

4. Eschenfeldt, P., Gamarnik, D.: Join the shortest queue with many servers. The heavy traffic asymptotics (2015). arXiv:1502.00999

5. Eschenfeldt, P., Gamarnik, D.: Supermarket queueing system in the heavy traffic regime. Short queue dynamics (2016). arXiv:1610.03522

6. Fairthorne, M.: PhD Thesis, London School of Economics (2011)

7. Graham, C.: Chaoticity on path space for a queuing network with selection of the shortest queue among several. J. Appl. Probab. 37, 198-201 (2000)

8. Graham, C.: Functional central limit theorems for a large network in which customers join the shortest of several queues. Prob. Theor. Relat. Fields 131, 97-120 (2004)

9. Luczak, M.J.: Concentration of measure and mixing of Markov chains. Discret. Math. Theor. Comp. Sci. (Proc. 5th Colloq. Mathem. Comp. Sci.) 95-120 (2008)

10. Luczak, M.J., McDiarmid, C.: On the power of two choices: balls and bins in continuous time. Ann. Appl. Probab. 15, 1733-1764 (2006)

11. Luczak, M.J., McDiarmid, C.: On the maximum queue length in the supermarket model. Ann. Probab. 34, 493-527 (2006)

12. Luczak, M.J., McDiarmid, C.: Asymptotic distributions and chaos for the supermarket model. Electron. J. Probab. 12, 75-99 (2007)

13. Luczak, M.J., Norris, J.R.: Strong approximation for the supermarket model. Ann. Appl. Probab. 15, 2038-2061 (2005)

14. Luczak, M.J., Norris, J.R.: Averaging over fast variables in the fluid limit for Markov chains: application to the supermarket model with memory. Ann. Appl. Probab. 23, 957-986 (2013)

15. Martin, J.B., Suhov, Y.M.: Fast Jackson networks. Ann. Appl. Probab. 9, 854-870 (1999)

16. Meyer, C.D.: Matrix Analysis and Applied Linear Algebra. SIAM, Philadelphia (2000)

17. Mitzenmacher, M.: Load balancing and density dependent jump Markov processes. In: Proceedings of 37th IEEE Annual Symposium on Foundations of Computer Science (FOCS), pp. 213-222 (1996)

18. Mitzenmacher, M.: The power of two choices in randomized load-balancing. PhD thesis, Berkeley. http://www.eec.harvard.edu michaelm/ (1996)

19. Mitzenmacher, M., Richa, A., Sitaraman, R.: The power of two random choices: a survey of techniques and results. In: Pardalos, P., Rajasekaran, S., Rolim, J. (eds.) Handbook of Randomized Computing: Volume 1, pp. 255-312 (2001)

20. Mitzenmacher, M., Prabhakar, B., Shah, D.: Load-balancing with memory. In: Proceedings of 43rd IEEE Annual Symposium on Foundations of Computer Science (FOCS), pp. 799-808 (2002)

21. Mukherjee, D., Borst, S.C., van Leeuwaarden, J.S.H., Whiting, P.A.: Universality of power-of-d load balancing in many server systems (2016). arXiv:1612.00723v1

22. Turner, S.R.E.: The effect of increasing routing choice on resource pooling. Probab. Eng. Inf. Sci. 12, 109-124 (1998)

23. Vvedenskaya, N.D., Dobrushin, R.L., Karpelevich, F.I.: Queueing system with selection of the shortest of two queues: an asymptotic approach. Prob. Inf. Transm. 32, 15-27 (1996) 